\title{
The Boundary Layer Equations and a Dimensional Split Method for Navier-Stokes Equations in Exterior Domain of a Spheroid and Ellipsoid
}

\author{
Jian Su', Hongzhou Fan'2, Weibing Feng3, Hao Chen', Kaitai Li' \\ ${ }^{1}$ School of Mathematics and Statistic, Xi'an Jiaotong University, Xi'an, China \\ ${ }^{2}$ School of Energy and Power Engineering, Xi'an Jiaotong University, Xi'an, China \\ ${ }^{3}$ School of Computer Engineering and Science, Shanghai University, Shanghai, China \\ ${ }^{4}$ School of Mechanics and Engineering, Southwest Jiaotong University, Chengdu, China \\ Email: ktli@mail.xjtu.edu.cn
}

Received 22 February 2015; accepted 15 March 2015; published 20 March 2015

Copyright (C) 2015 by authors and Scientific Research Publishing Inc.

This work is licensed under the Creative Commons Attribution International License (CC BY).

http://creativecommons.org/licenses/by/4.0/

(c) (i) Open Access

\begin{abstract}
In this paper, the boundary layer equations (abbreviation BLE) for exterior flow around an obstacle are established using semi-geodesic coordinate system ( $S$-coordinate) based on the curved two dimensional surface of the obstacle. BLE are nonlinear partial differential equations on unknown normal viscous stress tensor and pressure on the obstacle and the existence of solution of BLE is proved. In addition a dimensional split method for dimensional three Navier-Stokes equations is established by applying several 2D-3C partial differential equations on two dimensional manifolds to approach 3D Navier-Stokes equations. The examples for the exterior flow around spheroid and ellipsoid are presents here.
\end{abstract}

\section{Keywords}

Boundary Layer Equations, Dimensional Split Method, Navier-Stokes Equations, Dimensional Two Manifold

\section{Introduction}

In computational fluid dynamics, one need to compute the drag exerted on a body in flow field; in particular, optimal shape design has received considerable attention already, see Li and Huang [1], Li, Chen and Yu [2], 
and Li, Su, Huang [3]. It has become vast enough to branch into several disciplines on the theoretical side, many results deal with the existence of solutions to the problem or its relaxed form, on the practical side, topological shape $\mathfrak{I}$ optimization which solves numerically the relaxed problem or by local shape variation. In this case we have to compute the velocity gradient $u_{1}:=\frac{\partial u}{\partial n}$ along the normal to the surface of the boundary and normal stress tensor $\sigma_{n}$ to the surface. All those computation have to do in the boundary layer. Therefore this leads to make very fine mash; for example, $80 \%$ nodes will be concentred in a neighborhood of the surface of the body.

In this paper a boundary layer equations for $u_{1}, p_{0}=\left.p\right|_{\mathfrak{I}}$ on the surface will be established using local semi-geodesic coordinate system based on the surface, provide the computational formula for the drag functional. In addition, a dimensional split method for three dimensional Navier-Stokes equations is established by applying several 2D-3C partial differential equations on the two dimensional manifolds to approximate 3D Navier-Stokes equation.

The Dimensional Slitting Methods deal, for examples, with thin domain problem as elastic shell (see Ciarlet [4], Li, Zhang and Huang [5]), Temam and Ziane [6], and with boundary value problem with complexity boundary geometry (see [7]-[10]).

The content of the paper is organized as the followings. Section 2 establishes semi-geodesic coordinate system and related the Navier-Stokes equations; Section 3 assumes that the solutions of Navier-Stokes equations in the boundary layer can be made Taylor expansion with respect to transverse variable, derive the equations for the terms of Taylor expansion; Section 4 proves the existence of the solutions of the BLE; Section 5 provides the computational formula of the drag functional; Section 6 provide a dimensional splitting method for 3D Navier-Stokes equations; Section 7 provide some examples.

\section{Navier-Stokes Equations and Its Variational Formulation in a Semi-Geodesic Coordinate System}

Through this paper, we consider state steady incompressible Navier-Stokes equations and its variational formulation in a thin domain $\Omega_{\delta}$, a strip with thickness $\delta$ and by a Lipchsitz continuous boundary $\partial \Omega:=\Gamma=\Gamma_{0} \cup \Gamma_{1}$,

$$
\left\{\begin{array}{l}
-\mu \Delta \mathbf{u}+(\mathbf{u} \nabla) \mathbf{u}+\nabla p=\mathbf{f}, \\
\operatorname{divu}=0, \\
\left.\mathbf{u}\right|_{\Gamma_{0}}=0, \text { essential boundary condition, } \\
\left.\sigma \cdot \mathbf{n}\right|_{\Gamma_{1}}=\mathbf{h} \text {, Nature boundary condition, }
\end{array}\right.
$$

or

$$
\left\{\begin{array}{l}
-2 \mu \nabla_{j} e^{i j}(\mathbf{u})+(\mathbf{u} \nabla) \mathbf{u}^{i}+g^{i j} \nabla_{j} p=f^{i}, \\
\operatorname{divu}=0, \\
\left.\mathbf{u}\right|_{\Gamma_{0}}=0, \text { essential boundary condition, } \\
\left.\sigma \cdot \mathbf{n}\right|_{\Gamma_{1}}=\mathbf{h} \text {, Nature boundary condition, }
\end{array}\right.
$$

which are invariant form in any curvilinear coordinate system. Let

$$
\begin{aligned}
& \mathbf{V}\left(\Omega_{\delta}\right)=\left\{\mathbf{u} \in \mathbf{H}^{1}\left(\Omega_{\delta}\right),\left.\mathbf{u}\right|_{\Gamma_{0}}=0,\right\} \\
& \mathbf{M}\left(\Omega_{\delta}\right)=\left\{q \in L^{2}\left(\Omega_{\delta}\right), \int_{\Omega} q \mathrm{~d} x=0 \text { if meas } \Gamma_{1}=0,\right\}
\end{aligned}
$$

At first, we introduce semi-geodesic coordinate system (abbreviation $S$-coordinate). As well known thhat boundary layer $\Omega_{\delta} \in E^{3}$ in 3D Euclidean space bounded by $\Gamma_{b}=\mathfrak{I}$ and $\Gamma_{t}$ where $\mathfrak{I}=\cup_{\alpha} \mathfrak{I}_{\alpha}$ is bottom of the boundary layer, a surface of solid boundary of the flow fluid, and $\Gamma_{t}=\mathfrak{I}+\mathbf{n} \delta:=\mathfrak{I}(\delta)$ is a top boundary of $\Omega_{\delta}$, an artificial interface of the flow fluid where $\mathbf{n}$ is unit normal vector to $\mathfrak{I}$ and $\delta$ is a parameter, the 
thickness of the strip, the boundary layer. Assume that there exits a smooth immersion $\theta\left(x^{1}, x^{2}\right): \bar{D} \subset \mathfrak{R}^{2} \mapsto \mathfrak{I}_{\alpha} \in E^{3}$ such that $\forall\left(x^{1}, x^{2}\right) \in D, \mathbf{e}_{\alpha}=\frac{\partial \theta}{\partial x^{\alpha}}$ are linearly independent where $D \subset \mathfrak{R}^{2}$ is a Lipschitz domain with boundary $\gamma=\partial D$ and $\left(x^{1}, x^{2}\right)$ are parameters which are called Gaussian coordinate on the surface $\mathfrak{I}$. It is obvious that $\mathbf{e}_{\alpha}$ are basis. So the geometry of the surface $\mathfrak{I}$ is given by first fundamental form and second fundamental form and third fundamental form which coefficients are metric tensor $a_{\alpha \beta}=\mathbf{e}_{\alpha} \mathbf{e}_{\beta}$ and curvature tensor $b_{\alpha \beta}=-\mathbf{n}_{\alpha} \mathbf{e}_{\beta}$ and tensor $c_{\alpha \beta}=\mathbf{n}_{\alpha} \mathbf{n}_{\beta}$ respectively where $\mathbf{n}$ is unit normal vector to $\mathfrak{I}$

$$
\mathbf{n}=\frac{\mathbf{e}_{1} \times \mathbf{e}_{2}}{\left|\mathbf{e}_{1} \times \mathbf{e}_{2}\right|}=\frac{\mathbf{e}_{1} \times \mathbf{e}_{2}}{\sqrt{a}}, \quad a=\operatorname{det}\left(a_{\alpha \beta}\right)>0
$$

Their contravariant components $a^{\alpha \beta}, b^{\alpha \beta}, c^{\alpha \beta}$ are given by

$$
a^{\alpha \beta} a_{\beta \lambda}=\delta_{\lambda}^{\alpha}, \quad b^{\alpha \beta}=a^{\alpha \lambda} a^{\beta \sigma} b_{\lambda \sigma}, \quad c^{\alpha \beta}=a^{\alpha \lambda} a^{\beta \sigma} c_{\lambda \sigma}
$$

What's follows that we will frequently used the inverse matrix $\left(\hat{b}^{\alpha \beta}, \hat{c}^{\alpha \beta}\right)$ of $\left(b_{\alpha \beta}, c_{\alpha \beta}\right)$ :

$$
\hat{b}^{\alpha \beta} b_{\beta \lambda}=\delta_{\lambda}^{\alpha}, \quad \hat{c}^{\alpha \beta} c_{\beta \lambda}=\delta_{\lambda}^{\alpha}
$$

Now, assume that there exists an unique normal vector $\mathbf{n}$ to $P_{0}=\left(x^{1}, x^{2}\right) \in \mathfrak{J}$ from each point $P \in \Omega_{\delta}$ such that (see Figure 1)

$$
\overrightarrow{\mathbf{O P}}=\overrightarrow{\mathbf{O P}}_{0}+\mathbf{n}\left(x^{\alpha}\right) \xi, \quad 0 \leq \xi \leq \delta
$$

where $O$ is origin. Thereby, point $P$ is determined by triple numbers $\left(x^{\alpha}, \xi\right)$. Inversely, a triple numbers $\left(x^{\alpha}, \xi ;\left(x^{\alpha}\right) \in \omega, 0 \leq \xi \leq \delta\right)$ can determine uniquely a point $P \in \Omega_{\delta}$. Curvilinear coordinate $\left(x^{1}, x^{2}, \xi\right)$ in $E^{3}$ is called semi-geodesic coordinate based on the surface $\mathfrak{J}$. Its bases vectors are $\left(\mathbf{e}_{\alpha}, \mathbf{n}\right)$ and the metric tensor $g_{i j}$ of 3D Euclidean space $E^{3}$ in this semi-geodesic coordinate are given by

$$
\begin{aligned}
& g_{\alpha \beta}=\frac{\partial \Theta}{\partial x^{\alpha}} \frac{\partial \Theta}{\partial x^{\beta}}, \quad g_{3 \alpha}=g_{\alpha 3}=\frac{\partial \Theta}{\partial x^{\alpha}} \frac{\partial \Theta}{\partial \xi}, \quad g_{33}=\frac{\partial \Theta}{\partial \xi} \frac{\partial \Theta}{\partial \xi}, \\
& \text { contravariant components } g^{i j} g_{j k}=\delta_{k}^{i}, \\
& \Theta=\boldsymbol{\theta}+\boldsymbol{\xi} \mathbf{n},
\end{aligned}
$$

Therefore, the metric tensor of $E^{3}$ can be expressed by the metric tensor of $\mathfrak{I}$ in the semi-geodesic coordinate system:

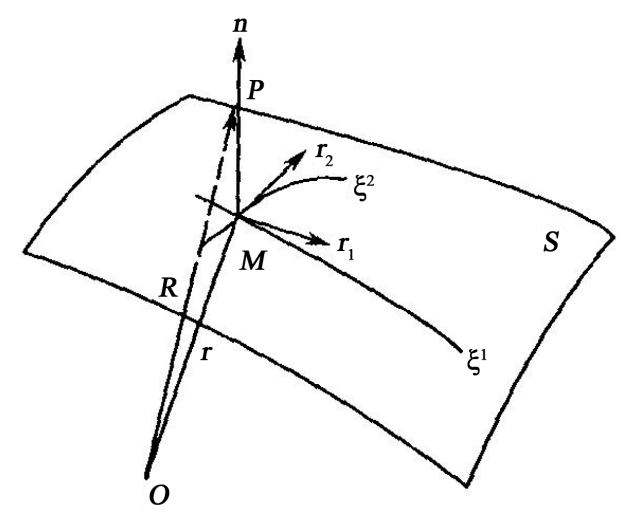

Figure 1. The diagram of semi-geodesic coordinate system. 


$$
\left\{\begin{array}{l}
g_{\alpha \beta}=\frac{\partial\left(\mathscr{R}\left(x^{\alpha}\right)+\xi \mathbf{n}\right)}{\partial x^{\alpha}} \frac{\partial\left(\mathscr{R}\left(x^{\alpha}\right)+\xi \mathbf{n}\right)}{\partial x^{\beta}}=a_{\alpha \beta}(x)-2 \xi b_{\alpha \beta}(x)+\xi^{2} c_{\alpha \beta}(x), \\
g_{\alpha 3}(x, \xi)=g_{3 \alpha}(x, \xi)=0, \quad g_{33}(x, \xi)=1, \\
g(x, \xi)=\operatorname{det}\left(g_{i j}\right)=\theta^{2} a(x), \quad a=\operatorname{det}\left(a_{\alpha \beta}\right), \\
g^{\alpha \beta}(x, \xi)=\theta^{-2}\left(a^{\alpha \beta}(x)-2 K \hat{b}^{\alpha \beta}(x) \xi+K^{2} \xi^{2} \hat{c}^{\alpha \beta}(x)\right)=\theta^{-2}\left(p(\xi) a^{\alpha \beta}+q(\xi) b^{\alpha \beta}\right), \\
g^{3 \alpha}(x, \xi)=g^{\alpha 3}(x, \xi)=0, \quad g^{33}(x, \xi)=1, \\
\theta=1-2 H \xi+K \xi^{2}, \\
p(\xi)=1-4 H \xi+\left(4 H^{2}-K\right) \xi^{2}, \quad q(\xi)=2 \xi-2 H \xi^{2} .
\end{array}\right.
$$

(see ref. [1]) wherre $H, K$ are mean curvature and Gaussian curvature of $\mathfrak{I}$. Throughout this paper, we employ semi-geodesic coordinate system $\left(x^{\alpha}, x^{3}:=\xi\right)$ based on the surface $\mathfrak{I}$ (see [1] and Figure 1) (later on, denote $S$-coordinate). The metric tensor of $E^{3}$ in this coordinate are denoted by $g_{i j}, g^{i j}$. It is obvious that the determinate $g=\operatorname{det}\left(g_{i j}\right)=a \theta^{2}>0$ if $\xi$ is small enough. Hence coordinate $\left(x^{\alpha}, \xi\right)$ is nonsingular.

In addition, we review the main notation. Greek indices and exponents belong to the set $\{1,2\}$, while Latin indices and exponents (except when otherwise indicated, as when they are used to index sequences) belong to the set $\{1,2,3\}$, and the summation convention with respect to repeated indices and exponents is systematically used. Symbols such as $\delta_{\beta}^{\alpha}$ or $\delta_{j}^{i}$ designate the Kronecker's symbol. The Euclidean scalar product and the the exterior product of $\mathbf{a}, \mathbf{b} \in E^{3}$ are noted $\mathbf{a} \cdot \mathbf{b}$ and $\mathbf{a} \times \mathbf{b}$; the Euclidean norm of $\mathbf{a} \in R^{3}$ is noted $|\mathbf{a}|$. Furthermore, the physical or geometric quantities with the asterisk $*$ express the quantities on the manifold $\mathfrak{I}$, for example, $\nabla_{\alpha}$ is covariant derivative on $\mathfrak{I}$. Furthermore, the physical or geometric quantities with the asterisk * express the quantities on the manifold $\mathfrak{I}$, for example, $\nabla_{\alpha}$ is covariant derivative on $\mathfrak{I}$. Furthermore, the notations $\varepsilon^{\alpha \beta}, \varepsilon_{\alpha \beta}$ are given contravariant components and covariant components of the permutation tensor on $\mathfrak{J}$

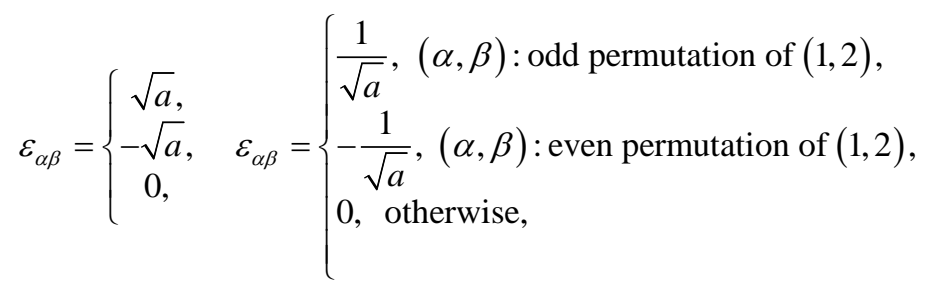

There are following relations of the first,second and third fundamental forms (ref. [1])

$$
\left\{\begin{array}{l}
K a_{\alpha \beta}-2 H b_{\alpha \beta}+c_{\alpha \beta}=0, \quad a^{\alpha \beta}-2 H \hat{b}^{\alpha \beta}+K \hat{c}^{\alpha \beta}=0 ; \\
2 H a^{\alpha \beta}-b^{\alpha \beta}-K \hat{b}^{\alpha \beta}=0 ; \\
\left(K-4 H^{2}\right) a^{\alpha \beta}+2 H b^{\alpha \beta}+K^{2} \hat{c}^{\alpha \beta}=0 ;
\end{array}\right.
$$

The following give the relations of differential operators in the space and on $\mathfrak{I}$ (see [1]). For example, under the $S$-coordinate system, the Christoffel symbols of $E^{3}$ and $\mathfrak{I}$ satisfy

$$
\Gamma_{\alpha \beta}^{\gamma}=\Gamma_{\alpha \beta}^{*}+\theta^{-1} R_{\alpha \beta}^{\gamma}, \quad \Gamma_{\beta 3}^{\alpha}=\theta^{-1} I_{\beta}^{\alpha}, \quad \Gamma_{\alpha \beta}^{3}=J_{\alpha \beta}, \Gamma_{\beta 3}^{3}=\Gamma_{3 \beta}^{3}=\Gamma_{33}^{\alpha}=\Gamma_{33}^{3}=0 .
$$

and covariant derivatives of the vector field are given by

$$
\begin{aligned}
& \nabla_{i} u^{j}=\frac{\partial u^{j}}{\partial x^{i}}+\Gamma_{i k}^{j} u^{k}, \quad \forall u \in H^{1}(\Omega)^{3} ; \quad \stackrel{*}{\nabla}_{\alpha} u^{\beta}=\frac{\partial u^{\beta}}{\partial x^{\alpha}}+\Gamma^{\beta}{ }_{\alpha \lambda} u^{\lambda}, \forall u \text { on } T \Im ; \\
& \operatorname{div}(u)=\nabla_{i} u^{j}=\frac{1}{\sqrt{g}} \frac{\partial}{\partial x^{i}}\left(\sqrt{g} u^{i}\right) ; \quad \operatorname{div}^{*} u=\stackrel{\nabla}{\nabla}_{\alpha} u^{\alpha}=\frac{1}{\sqrt{a}} \frac{\partial}{\partial_{x^{\alpha}}}\left(\sqrt{a} u^{\alpha}\right) ; \\
& \operatorname{rot}(u)=\nabla \times u=\varepsilon^{i j k} \nabla_{j} u_{k} \mathbf{e}_{i} ; \quad \stackrel{\operatorname{rot}}{\operatorname{rot}}(u)=\varepsilon^{\alpha \beta} \stackrel{*}{\nabla}_{\alpha} u_{\beta} .
\end{aligned}
$$


where $u_{i}=g_{i j} u^{j}$ is covariant component of vector $\mathbf{u}$. The strain tensor of vector field in $\mathfrak{R}^{3}$ and on $\mathfrak{I}$ are given by respectively

$$
\begin{aligned}
& e_{i j}(u)=\frac{1}{2}\left(\nabla_{i} u_{j}+\nabla_{j} u_{i}\right)=\frac{1}{2}\left(\delta_{i}^{k} g_{j l}+\delta_{j}^{k} g_{i l}\right) \nabla_{k} u^{l} ; \\
& \stackrel{*}{e}_{\alpha \beta}(u)=\frac{1}{2}\left(\stackrel{*}{\nabla}_{\alpha} u_{\beta}+\stackrel{*}{\nabla}_{\beta} u_{\alpha}\right)=\frac{1}{2}\left(\delta_{\alpha}^{\lambda} a_{\beta \sigma}+\delta_{\beta}^{\lambda} a_{\alpha \sigma}\right) \stackrel{*}{\nabla}_{\lambda} u^{\sigma} ;
\end{aligned}
$$

Of course, $\operatorname{div}(u)=g^{i j} e_{i j}(u) ; \operatorname{div}(u)=a^{\alpha \beta} \stackrel{e}{\alpha \beta}_{\alpha \beta}(u)$. Under the $S$-coordinate system there are following formula for the covariant derivatives of the vectors in the space $\mathfrak{R}$ and on the $\mathfrak{I}$ (ref. [1])

$$
\left\{\begin{array}{l}
\nabla_{\alpha} u^{\beta}=\stackrel{*}{\nabla}_{\alpha} u^{\beta}+\theta^{-1}\left(I_{\alpha}^{\beta} u^{3}+R_{\alpha \lambda}^{\beta} u^{\lambda}\right), \quad \nabla_{\alpha} u^{3}=\frac{\partial u^{3}}{\partial x^{\alpha}}+J_{\alpha \beta} u^{\beta}, \\
\nabla_{3} u^{\beta}=\frac{\partial u^{\beta}}{\partial \xi}+\theta^{-1} I_{\alpha}^{\beta} u^{\alpha}, \quad \nabla_{3} u^{3}=\frac{\partial u^{3}}{\partial \xi}, \\
\operatorname{div} u=\operatorname{div}(u)+\frac{\partial u^{3}}{\partial \xi}+\theta^{-1}\left[-2 H u^{3}+\left(2 K u^{3}-2 u^{\lambda} \nabla_{\lambda} H\right) \xi+u^{\lambda} \nabla_{\lambda} K \xi^{2}\right] \\
I_{\beta}^{\alpha}=-b_{\beta}^{\alpha}+K \xi \delta_{\beta}^{\alpha}, \quad J_{\alpha \beta}=b_{\alpha \beta}-\xi c_{\alpha \beta}, \\
R_{\beta \sigma}^{\alpha}=-\stackrel{*}{\nabla}_{\sigma} b_{\beta}^{\alpha} \xi+K \hat{b}_{\mu}^{\alpha} \stackrel{*}{\nabla}_{\sigma} b_{\beta}^{\mu} \xi^{2}=-\stackrel{*}{\nabla} \sigma_{\sigma}^{\alpha} \xi+\left(2 H \delta_{\mu}^{\alpha}-b_{\mu}^{\alpha}\right) \stackrel{*}{\nabla}_{\sigma} b_{\beta}^{\mu} \xi^{2} .
\end{array}\right.
$$

The strain tensors of the vectors in $\mathfrak{R}$ and on $\mathfrak{J}$ can be expressed as

$$
\left\{\begin{array}{l}
e_{\alpha \beta}(u)=\frac{1}{2}\left(\nabla_{\alpha} u_{\beta}+\nabla_{\beta} u_{\alpha}\right)=\gamma_{\alpha \beta}(u)+\gamma_{\alpha \beta}(u) \xi+{ }^{2} \gamma_{\alpha \beta}(u) \xi^{2} \\
e_{\alpha 3}(u)=\frac{1}{2}\left(g_{\alpha \beta} \frac{\partial u^{\beta}}{\partial \xi}+\nabla_{\alpha} u^{3}\right)=\gamma_{\alpha 3}(u)+\gamma_{\alpha 3}(u) \xi^{2} \gamma_{\alpha 3}(u) \xi^{2} \\
e_{33}(u)=\nabla_{3} u^{3}=\frac{\partial u^{3}}{\partial \xi}
\end{array}\right.
$$

where

$$
\left\{\begin{array}{l}
\gamma_{\alpha \beta}(\mathbf{u})=\stackrel{*}{\alpha \beta}_{\alpha \beta}(\mathbf{u})-b_{\alpha \beta} u^{3}, \\
1_{\alpha \beta}(\mathbf{u})=-\left(b_{\alpha \lambda} \stackrel{*}{\nabla}_{\beta} u^{\lambda}+b_{\beta \lambda} \stackrel{*}{\nabla}_{\alpha} u_{\lambda}\right)+c_{\alpha \beta} u^{3}-\stackrel{*}{\nabla}_{\lambda} b_{\alpha \beta} u^{\lambda} ; \\
2 \\
\gamma_{\alpha \beta}(\mathbf{u})=\frac{1}{2}\left(c_{\alpha \lambda} \stackrel{*}{\nabla}_{\beta} u^{\lambda}+c_{\beta \lambda} \stackrel{*}{\nabla}_{\alpha} u^{\lambda}+\stackrel{*}{\nabla}_{\lambda} c_{\alpha \beta} u^{\lambda}\right) \\
\gamma_{\alpha 3}(\mathbf{u})=\gamma_{3 \alpha}(\mathbf{u})=\frac{1}{2}\left(a_{\alpha \beta} \frac{\partial u^{\beta}}{\partial \xi}+\stackrel{*}{\nabla}_{\alpha} u^{3}\right) \\
1 \\
\gamma_{\alpha 3}(\mathbf{u})=-b_{\alpha \beta} \frac{\partial u^{\beta}}{\partial \xi}, \quad \stackrel{2}{\alpha 3}^{2}(\mathbf{u})=\frac{1}{2} c_{\alpha \beta} \frac{\partial u^{\beta}}{\partial \xi} \\
\gamma_{33}(\mathbf{u})=\frac{\partial u^{3}}{\partial \xi}, \quad \gamma_{33}(\mathbf{u})=\stackrel{2}{\gamma}_{33}(\mathbf{u})=0 . \\
\gamma^{\alpha \beta}(\mathbf{u})=a^{\alpha v} a^{\beta \sigma} \gamma_{v \sigma}(\mathbf{u}) .
\end{array}\right.
$$

In the semi-geodesic coordinate system (see next section), define the bilinear form $a(\cdot, \cdot)$ and trilinear form $b(\cdot, \cdot$, 


$$
\left\{\begin{array}{l}
a(\mathbf{u}, \mathbf{v})=\int_{\Omega_{\delta}} \mu \nabla \mathbf{u} \nabla \mathbf{v} \mathrm{d} V=\int_{\Omega_{\delta}} \mu g^{i j} g_{k m} \nabla_{i} u^{m} \nabla_{j} v^{k} \sqrt{g} \mathrm{~d} x, \\
\text { or } \\
a(\mathbf{u}, \mathbf{v})=\int_{\Omega_{\delta}} 2 \mu e^{i j}(\mathbf{u}) e_{i j}(\mathbf{v}) \sqrt{g} \mathrm{~d} x \mathrm{~d} \xi=\int_{D} 2 \mu g^{i k} g^{j m} e_{k m}(\mathbf{u}) e_{i j}(\mathbf{v}) \theta \sqrt{a} \mathrm{~d} x, \\
b(u ; u, v)=\int_{\Omega_{\delta}} u^{j} \nabla_{j} u^{i} g_{i k} v^{k} \sqrt{g} \mathrm{~d} x, \\
\langle\mathbf{F}, \mathbf{v}\rangle=(\mathbf{f}, \mathbf{v})+\langle\boldsymbol{\sigma} \cdot \mathbf{n}, \mathbf{v}\rangle=(\mathbf{f}, \mathbf{v})+\left.\langle\mathbf{h}, \mathbf{v}\rangle\right|_{\Gamma_{1}},
\end{array}\right.
$$

Then, the primitive variable variational formulation for Navier-Stokes Equations (2.1') is given by

$$
\left\{\begin{array}{l}
\text { Find }(\mathbf{u}, p) \in V(\Omega) \times M(\Omega) \text { such that } \\
a(\mathbf{v}, \mathbf{v})+b(\mathbf{u}, \mathbf{u}, \mathbf{v})-(p, \operatorname{div} \mathbf{v})=(\mathbf{F}, \mathbf{v}), \quad \forall \mathbf{v} \in V(\Omega), \\
(q, \operatorname{divu})=0, \quad \forall q \in M(\Omega),
\end{array}\right.
$$

while the Navier-Stokes Equations (2.7) in semi-geodesic coordinate system are expressed as

$$
\begin{aligned}
& \int-\mu\left[\frac{\partial^{2} u^{\alpha}}{\partial \xi^{2}}+4 \theta^{-1} I_{\gamma}^{\alpha} \frac{\partial u^{\gamma}}{\partial \xi}+g^{\alpha \beta} \stackrel{*}{\nabla}_{\beta} \frac{\partial u^{3}}{\partial \xi}\right]+u^{3} \frac{\partial u^{\alpha}}{\partial \xi}+\mathcal{L}^{\alpha}(\mathbf{u})+g^{\alpha \beta} \stackrel{*}{\nabla}_{\beta} p+u^{\beta} \stackrel{*}{\nabla}_{\beta} u^{\alpha} \\
& +2 \theta^{-1} I_{\beta}^{\alpha} u^{\beta} u^{3}+\theta^{-1} R_{\beta \sigma}^{\alpha} u^{\beta} u^{\sigma}=f^{\alpha}, \\
& \left\{-2 \mu\left[\frac{\partial^{2} u^{3}}{\partial \xi^{2}}-2 \theta^{-1}(H-K \xi) \frac{\partial u^{3}}{\partial \xi}+\frac{1}{2} \frac{\partial}{\partial \xi} \operatorname{div}^{*} \mathbf{u}+\frac{1}{2} g^{\lambda \sigma}\left(\stackrel{*}{\nabla}_{\lambda} g_{\beta \sigma}-g_{\beta \mu} \Theta^{-1} R_{\lambda \sigma}^{\mu}\right) \frac{\partial u^{\beta}}{\partial \xi}\right]+\frac{\partial p}{\partial \xi}+u^{3} \frac{\partial u^{3}}{\partial \xi}\right. \\
& +\mathcal{L}^{3}(\mathbf{u})+u^{\beta} \stackrel{*}{\nabla}_{\beta} u^{3}+J_{\beta \sigma} u^{\beta} u^{\sigma}=f^{3}, \\
& \operatorname{divu}=\frac{\partial u^{3}}{\partial \xi} \operatorname{div}^{*} \mathbf{u}+\theta^{-1}\left[-2 H u^{3}+2\left(K u^{3}-u^{\beta} \stackrel{*}{\nabla}_{\beta} H\right) \xi+u^{\beta} \stackrel{*}{\nabla}_{\beta} K \xi^{2}\right]=0, \\
& \left\{\begin{array}{l}
\mathcal{L}^{\alpha}(\mathbf{u}):=-2 \mu\left[g^{\alpha \beta} g^{\lambda \sigma} \sum_{k=0}^{2}\left(\stackrel{*}{\nabla}_{\lambda}{ }^{k} \gamma_{\beta \sigma}(\mathbf{u})-2 \Theta^{-1} R_{\lambda \beta}^{v}{ }_{\beta \sigma}^{k} \gamma_{\beta \sigma}(\mathbf{u})\right) \xi^{k}+\theta^{-1} g^{\alpha \beta} I_{\beta}^{\sigma} \stackrel{\nabla}{\nabla}_{\sigma} u^{3}\right], \\
\mathcal{L}^{3}(\mathbf{u})=-2 \mu\left[\frac{1}{2} g^{\lambda \sigma} \stackrel{*}{\nabla}_{\lambda}^{*} \nabla_{\sigma} u^{3}-\theta^{-1} g^{\lambda \sigma} I_{\lambda}^{\beta} \sum_{k=0}^{2}{ }_{\beta \sigma}^{k}(\mathbf{u}) \xi^{k}-\frac{1}{2} g^{\lambda \sigma} \theta^{-1} R_{\lambda \sigma}^{\beta} \stackrel{*}{\nabla}_{\beta} u^{3}\right],
\end{array}\right.
\end{aligned}
$$

\section{Boundary Layer Equations}

Assume that $\mathfrak{I}$ is a two dimensional manifold parameterized by $\mathcal{R}(x)$. In the neighborhood of the orientate surface $\mathfrak{I}$ let define a surface $\mathfrak{I}(\delta)$ :

$$
\mathfrak{I}(\delta)=\left\{\mathscr{S}(x)=\mathscr{h}(x)+\delta \mathbf{n}(x), \forall x \in D \subset \mathbf{E}^{2}\right\}, \mathbf{n} \text { is unit outward normal vector to } \mathfrak{I}
$$

It is obvious that $\mathfrak{I}(\delta)$ ia a geodesic parallel surface of $\mathfrak{I}$ and the geodesic distance each other is equal to $\delta$ where $\delta$ is a small constant.In this paper we only consider exterior flow around a body occupied by $\Omega$ with a two dimensional manifold $\mathfrak{I}=\partial \Omega$ without boundary. The boundary layer domain

$$
\Omega_{\delta}=\{\mathbf{R}(x)=\mathscr{R}(x)+\xi \mathbf{n}, 0 \leq \xi \leq \delta, \forall x \in D\} \subset \mathbf{E}^{3} \text { bounded by } \mathfrak{I} \cup \mathfrak{I}(\delta)
$$

Domain $\Omega_{\delta}$ is called the "stream layer".

Assumption AI assume that the solutions $(\mathbf{u}, p)$ of Navier-Stokes Equation (2.7) in boundary layer $\Omega_{\delta}$ in semi-geodesic coordinate system and right term $f$ can be made Taylor expansion with respect to the transverse variable $0 \leq \xi \leq \delta$

$$
\left\{\begin{array}{l}
\mathbf{u}(x, \xi)=\mathbf{u}_{1}(x) \xi+\mathbf{u}_{2}(x) \xi^{2}+\cdots \\
p(x, \xi)=p_{0}(x)+p_{1}(x) \xi+p_{2}(x) \xi^{2}+\cdots \\
\mathbf{f}(x, \xi)=\mathbf{f}_{0}(x)+\mathbf{f}_{1}(x) \xi+\mathbf{f}_{2}(x) \xi^{2}+\cdots
\end{array}\right.
$$


In same time, the test vector also can be made Taylor expansion

$$
\mathbf{v}=\mathbf{v}_{1}(x) \xi+\mathbf{v}_{2}(x) \xi^{2}+\cdots, \quad q=q_{0}(x)+q_{1}(x) \xi+q_{2}(x) \xi^{2}+\cdots
$$

Theorem 1 In a boundary layer domain $\Omega_{\delta}$ with non-slip boundary condition $\mathbf{u}_{0}=0$, if the Assumption AI (3.1) is satisfied, then nine unknown of $\left(\mathbf{u}_{1}, \mathbf{u}_{2}, p_{0}, p_{1}, p_{2}\right)$ satisfy following a system of three partial differential equations which are called boundary layer equations I (BLE I):

$$
\begin{aligned}
& \int-\frac{\mu \delta^{3}}{3}\left[\stackrel{*}{\Delta} u_{1}^{\alpha}+a^{\alpha \lambda} \stackrel{*}{\nabla}_{\lambda} \operatorname{div} \mathbf{u}_{1}+K u_{1}^{\alpha}\right]+a^{\alpha \beta} \eta_{\beta \sigma} u_{1}^{\sigma}-\frac{\delta^{3}}{3} a^{\alpha \beta} \stackrel{*}{\nabla}_{\beta} p_{1}+\frac{\delta^{4}}{4}\left(u_{1}^{\lambda} \stackrel{*}{\nabla}_{\lambda} u_{1}^{\alpha}-u_{1}^{\alpha} \operatorname{div}^{*} \mathbf{u}_{1}\right) \\
& +\frac{2 H \delta^{3}}{3} a^{\alpha \beta} \nabla_{\beta}^{*} p_{0}=\mathscr{F}^{\alpha}\left(\mathbf{u}_{2}\right) \\
& -\frac{\delta^{3}}{3} \Delta p_{0}+M\left(\mathbf{u}_{1}\right)=F_{p}, \quad u_{1}^{3}=0 \\
& \left(\left.\left(\mathbf{u}_{1}, p_{0}\right)\right|_{\partial D}\right. \text { satisfies periodic boundary condition. }
\end{aligned}
$$

and five algebraic equations

$$
\left\{\begin{array}{l}
\frac{2 \mu \delta^{3}}{3} u_{2}^{\alpha}=a^{\alpha \beta} F_{\beta}^{2}-a^{\alpha \beta} q_{\beta \sigma} u_{1}^{\sigma}+\frac{\delta^{3}}{3} a^{\alpha \beta} \stackrel{*}{\nabla}_{\beta} p_{0}, \\
2 u_{2}^{3}+\operatorname{div}\left(\mathbf{u}_{1}\right)=0 \\
\frac{\delta^{3}}{3} p_{2}+\left(\frac{\delta^{2}}{2}-\frac{4 H \delta^{3}}{3}\right) p_{1}+\kappa_{1} p_{0}+\frac{\delta^{4}}{4} b_{\alpha \beta} u_{1}^{\alpha} u_{1}^{\beta}-\frac{2 \mu \delta^{3}}{3} \beta_{0}\left(\mathbf{u}_{1}\right)-\frac{\mu}{2} \operatorname{div}\left(\left(\frac{\delta^{2}}{2}+\frac{2 H \delta^{3}}{3}\right) \mathbf{u}_{1}\right) \\
\quad-\mu\left(\frac{2 \delta^{2}}{2}+\frac{7 H \delta^{3}}{3}\right) \operatorname{div}_{1}^{*}=F_{3}^{1}, \\
\frac{2 \delta^{3}}{3} p_{1}+2\left(\frac{\delta^{2}}{2}-\frac{3 H \delta^{3}}{3}\right) p_{0}+\frac{9}{2} \frac{\mu \delta^{3}}{3} \operatorname{div}_{1}^{*}=F_{3}^{2},
\end{array}\right.
$$

Associated variational formulations with (3.2) is given by

$$
\left\{\begin{array}{l}
\text { Find }\left(\mathbf{u}_{1}, p_{0}\right) \in V(D) \times M(D) \text { such that } \frac{\delta^{3}}{3} a\left(\mathbf{u}_{1}, \mathbf{v}_{1}\right)+\left(\eta_{\alpha \beta} u_{1}^{\alpha}, v_{1}^{\beta}\right)+\frac{\delta^{4}}{4}\left(a_{\alpha \beta}\left(u_{1}^{\lambda} \nabla_{\lambda}^{*} u_{1}^{\alpha}-u_{1}^{\alpha} \operatorname{div}_{1}^{*}\right), v_{1}^{\beta}\right) \\
+\frac{\delta^{3}}{3}\left(p_{1}, \operatorname{div}_{1}\right)+\left(\frac{2 H \delta^{3}}{3} \nabla_{\beta}^{*} p_{0}, v_{1}^{\beta}\right)=\left(\mathscr{F}^{\alpha}\left(\mathbf{u}_{2}\right), a_{\alpha \beta} v^{\beta}\right), \quad \forall \mathbf{v}_{1} \in V(D) \\
\frac{\delta^{3}}{3}\left(\nabla p_{0}, \nabla q\right)+\left(M\left(\mathbf{u}_{1}\right), q\right)=\left(F_{p}, q\right), \quad \forall q \in M(D)
\end{array}\right.
$$

where the bilinear forms defined by

$$
\begin{aligned}
& a(\mathbf{u}, \mathbf{v}):=\left(a_{0}^{\alpha \beta \lambda \sigma} \stackrel{*}{e}_{\alpha \lambda}(\mathbf{u}),{ }^{*} e_{\alpha \lambda}(\mathbf{v})\right) \\
& (\cdot, \cdot)=\int_{\mathfrak{J}} \cdot \times \cdot \sqrt{a} \mathrm{~d} x
\end{aligned}
$$

and

$$
\left\{\begin{array}{l}
\eta_{\alpha \beta}=\frac{\mu}{2}\left[\left(\delta-\frac{2 H \delta^{2}}{2}\right) a_{\alpha \beta}+\left(\frac{6 H \delta^{3}}{3}-\frac{2 \delta^{2}}{2}\right) b_{\alpha \beta}\right], q_{\alpha \beta}=\frac{\mu}{2}\left[\left(\frac{\delta^{2}}{2}-\frac{4 H \delta^{3}}{3}\right) a_{\alpha \beta}-\frac{4 \delta^{3}}{3} b_{\alpha \beta}\right], \\
M\left(\mathbf{u}_{1}\right)=\frac{\mu}{2}\left(\frac{\delta^{2}}{2}+\frac{12 H \delta^{3}}{3}\right) \operatorname{div}^{*} \mathbf{u}_{1}-10 \mu \operatorname{div}^{*}\left(H \mathbf{u}_{1}\right)-\frac{2 \mu \delta^{3}}{3} b_{\beta}^{\alpha} \stackrel{*}{\nabla}_{\alpha} u_{1}^{\beta}, F_{p}=a^{\alpha \beta} \stackrel{*}{\nabla}_{\alpha} F_{\beta}^{2}, \\
\mathscr{F}^{\alpha}\left(\mathbf{u}_{2}\right)=-a^{\alpha \beta}\left[\frac{\mu}{2}\left[\left(\frac{\delta^{2}}{2}+\frac{4 H \delta^{3}}{3}\right) a_{\alpha \beta}+\frac{4 \delta^{3}}{3} b_{\alpha \beta}\right] u_{2}^{\alpha}+\frac{\mu}{2} \frac{\delta^{3}}{3} \stackrel{*}{\nabla}_{\beta} u_{2}^{3}\right]+a^{\alpha \beta} F_{\beta}^{1},
\end{array}\right.
$$


where

$$
\mathbf{F}^{1}=\mathbf{h} \delta+\frac{\mathbf{f}_{0} \delta^{2}}{2}+\frac{\mathbf{f}_{1} \delta^{3}}{3}, \quad \mathbf{F}^{2}=\mathbf{h} \delta^{2}+\frac{\mathbf{f}_{0} \delta^{3}}{3}
$$

$\mathbf{h}$ is normal stress tensor at $\Gamma_{t}$ (top boundary of boundary layer), $\mathbf{f}_{1}$ are defined by (3.1).

Next, let consider interface equation. In this case $\mathfrak{I}$ is a flexible surface (slip and passing through conditions).

Assumption AII Assume that the solutions $(\mathbf{u}, p)$ of Navier-Stokes Equation (2.1) in stream layer $\Omega_{ \pm 1 / 2}=\left\{\mathbf{R}(x)=\mathscr{h}(x)+\xi \mathbf{n},-\frac{1}{2} \leq \xi \leq \frac{1}{2}, x \in D\right\}$ in semi-geodesic coordinate system based on $\mathfrak{I}$ and right term $\mathbf{f}$ can be made Taylor expansion with respect to the transverse variable $\xi$

$$
\left\{\begin{array}{l}
\mathbf{u}(x, \xi)=\mathbf{u}_{0}(x)+\mathbf{u}_{1}(x) \xi+\mathbf{u}_{2}(x) \xi^{2}+\cdots \\
p(x, \xi)=p_{0}(x)+p_{1}(x) \xi+p_{2}(x) \xi^{2}+\cdots \\
\mathbf{f}(x, \xi)=\mathbf{f}_{0}(x)+\mathbf{f}_{1}(x) \xi+\mathbf{f}_{2}(x) \xi^{2}+\cdots
\end{array}\right.
$$

Theorem 2 Assume that the Assumption II is satisfied. Then six unknown of $\left(\mathbf{u}_{k}, p_{k}, k=0,1,2\right)$ in (3.7) satisfy following system of the nonlinear partial differential equations which are called stream layer equations II (abbreviation SLE II) (interface equations):

$$
\begin{aligned}
& \int-\mu \delta\left[\left({ }^{*} u_{0}^{\alpha}+a^{\alpha \lambda} \stackrel{*}{\nabla}_{\lambda} \operatorname{div}^{*} \mathbf{u}_{0}+K u_{0}^{\alpha}\right)+4 a^{\alpha \lambda} \stackrel{*}{\nabla}_{\lambda} H u_{0}^{3}+2 b^{\alpha \beta} \stackrel{*}{\nabla}_{\beta} u_{0}^{3}\right]+\delta\left(u_{0}^{\lambda} \stackrel{*}{\nabla}_{\lambda} u_{0}^{\beta}+u_{1}^{\beta} u_{0}^{3}\right)-\delta a^{\alpha \beta} \stackrel{*}{\nabla}_{\alpha} p_{0}=a^{\alpha \beta} F_{\alpha}^{0}, \\
& \left\{-2 \mu \delta \stackrel{*}{\Delta} u_{0}^{3}+2 \mu \delta\left(4 H^{2}-2 K\right) u_{0}^{3}+\delta\left[u_{0}^{\lambda} \stackrel{*}{\nabla} \lambda u_{0}^{3}+b_{\lambda \sigma} u_{0}^{\lambda} u_{0}^{\sigma}\right]-2 H \delta p_{0}-2 \mu \delta b_{\beta}^{\alpha} \stackrel{*}{\nabla} u_{\alpha}^{\beta}-\mu \delta \operatorname{div}_{0}^{*} \mathbf{u}_{1}=F_{3}^{0},\right. \\
& \operatorname{div}_{0}^{*} \mathbf{u}_{0}-2 H u_{0}^{3}+u_{1}^{3}=0, \\
& \mu\left(\left(\delta-\frac{H \delta^{2}}{2}\right) \delta_{\beta}^{\alpha}-\frac{\delta^{2}}{2} b_{\beta}^{\alpha}\right) u_{1}^{\beta}+\frac{\mu \delta^{2}}{2} a^{\alpha \beta} \stackrel{*}{\nabla}_{\beta} u_{1}^{3}-\frac{\mu \delta^{2}}{2}\left[\left(\Delta u_{0}^{\alpha}+a^{\alpha \lambda} \stackrel{*}{\nabla}_{\lambda} \operatorname{div}_{0}^{*}+K u_{0}^{\alpha}\right)\right. \\
& \left.+4 a^{\alpha \lambda} \stackrel{*}{\nabla}_{\lambda} H u_{0}^{3}+2 b^{\alpha \beta} \stackrel{*}{\nabla}_{\beta} u_{0}^{3}\right]+\frac{\delta^{2}}{2} a^{\alpha \beta} \stackrel{*}{\nabla}_{\beta} p_{0}+\frac{\delta^{2}}{2}\left(u_{0}^{\lambda} \stackrel{*}{\nabla}_{\lambda} u_{0}^{\alpha}+u_{1}^{\alpha} u_{0}^{3}-2 b_{\beta}^{\alpha} u_{0}^{\beta} u_{0}^{3}\right)=a^{\alpha \beta} F_{\beta}^{1}, \\
& -\frac{\delta^{2}}{2} p_{1}-\left(\delta-\frac{4 H \delta^{2}}{2}\right) p_{0}-\frac{\mu \delta^{2}}{2} \beta_{0}\left(\mathbf{u}_{0}\right)-\frac{\mu \delta^{2}}{2} \stackrel{*}{\Delta} u_{0}^{3}+\frac{\delta^{2}}{2}\left(u_{0}^{\lambda} \stackrel{*}{\nabla} \lambda u_{0}^{3}+b_{\alpha \beta} u_{0}^{\alpha} u_{0}^{\beta}\right)=F_{3}^{1}, \\
& \operatorname{div}^{*}\left(\mathbf{u}_{1}-2 H \mathbf{u}_{0}\right)+2 K u_{0}^{3}-4 H u_{1}^{3}=0 \text {, }
\end{aligned}
$$

The right terms

$$
\left\{\begin{array}{l}
F_{\beta}^{0}=a_{\alpha \beta}\left(h_{t}^{\alpha}-h_{b}^{\alpha}\right), \quad F_{3}^{0}=h_{t}^{3}-h_{b}^{3}, \quad F_{\beta}^{1}=a_{\alpha \beta} h_{t}^{\alpha} \delta, \quad F_{3}^{1}=\delta h_{t}^{3}, \\
h_{b}^{\alpha}=\left.\mu u_{1}^{\alpha}\right|_{\mathfrak{I}\left(-\frac{1}{2} \delta\right)}, \quad h_{b}^{3}=-\left.p_{0}\right|_{\mathfrak{I}\left(-\frac{1}{2} \delta\right)}, \\
h_{t}^{3}=\left.\left(-p_{0}+2 \mu u_{1}^{3}\right)\right|_{\mathfrak{S}\left(\frac{1}{2} \delta\right)}, \quad h_{t}^{\alpha}=\left.\mu\left(u_{1}^{\alpha}+a^{\alpha \beta} \stackrel{\nabla}{\beta}_{\beta} u_{0}^{3}-b_{\beta}^{\alpha} u_{0}^{\beta}\right)\right|_{\mathfrak{I}\left(\frac{1}{2} \delta\right)},
\end{array}\right.
$$

In particular, for flexible (slip condition $u_{0} \neq 0$ ) boundary surface $\mathfrak{I}$, neglect hight order terms and keep one order term of $\delta$, then (3.3) (3.4) and (3.5) become

The Proof of Theorems 1 and 2 is neglected. 


\section{The Existence of the Solution}

In this section we prove the existence of the weak solution of (3.2). To do that we consider variational formulation of (3.2). Let $V(\mathfrak{I})=H_{p}^{1}(D) \times H_{p}^{1}(D)$ where $H_{p}^{1}(D)$ is a sobolev space of 1-order with periodic boundary condition. Since ([14], Th.1.8.6) we claim

$$
\frac{1}{2}\left[{ }^{*} u_{1}^{\alpha}+a^{\alpha \lambda} \nabla_{\lambda}^{*} \operatorname{div}^{*} \mathbf{u}_{1}-K u_{1}^{\alpha}\right]=\nabla_{\beta}^{*} e^{* \beta}\left(\mathbf{u}_{1}\right)
$$

where

$$
e^{\alpha \beta}\left(\mathbf{u}_{1}\right)=\frac{1}{2}\left(a^{\alpha \lambda} \stackrel{*}{\nabla} \lambda u_{1}^{\beta}+a^{\beta \lambda} \stackrel{*}{\nabla} \lambda u_{1}^{\alpha}\right)
$$

Let define bilinear form: $\forall u, v \in V(\mathfrak{I})$,

$$
\left\{\begin{aligned}
& a(\mathbf{u}, \mathbf{v})=\frac{\delta^{3}}{32} \mu\left(e^{* \beta}(\mathbf{u}), e_{\alpha \beta}^{*}(\mathbf{v})\right)=\frac{\delta^{3}}{32} \mu\left(a^{\alpha \lambda} a^{\beta \sigma}{ }^{*} e_{\lambda \sigma}(\mathbf{u}), e_{\alpha \beta}^{*}(\mathbf{v})\right)+\left(Q_{\alpha \beta} u^{\alpha}, v^{\beta}\right) \\
&= \frac{\delta^{3}}{3}\left(a_{0}^{\alpha \beta \lambda \sigma}{ }^{*} e_{\lambda \sigma}(\mathbf{u}), e_{\alpha \beta}(\mathbf{v})\right)+\left(Q_{\alpha \beta} u_{1}^{\alpha}, v_{1}^{\beta}\right), \\
& a_{0}(p, q)=\frac{\delta^{3}}{3}\left(\stackrel{*}{\nabla}^{*}, \stackrel{*}{\nabla} q\right)=\frac{\delta^{3}}{3}\left(a^{\alpha \beta} \stackrel{*}{\alpha}_{\alpha} p, \stackrel{\nabla}{\beta}_{\beta} q\right), \quad \forall p, q \in L^{2}(D), \\
& \mathscr{L}(U, V)=\alpha_{0} a(\mathbf{u}, \mathbf{v})+\beta_{0} a_{0}(p, q), \quad \forall U, V \in V(D) \times L^{2}(D),
\end{aligned}\right.
$$

where $\left(\alpha_{0}, \beta_{0}\right)$ are two positive constants and $U=(\mathbf{u}, p), V=(\mathbf{v}, q)$ and

$$
(\cdot, \cdot)=\int_{D}\{\because \cdot\} \sqrt{a} \mathrm{~d} x
$$

Then corresponding variational formulation for (3.2) is given by

$$
\left\{\begin{array}{l}
\text { Find } U=\left(u_{1}^{\alpha}, \alpha=1,2, p_{0}\right) \in V(D) \times L^{2}(D) \text { such that } \\
\mathscr{L}(U, V)+\alpha_{0} \mathscr{\beta}\left(\mathbf{u}_{1}, \mathbf{u}_{1}, \mathbf{v}\right)+\alpha_{0}\left(\mathscr{O}_{\beta}\left(p_{0}\right), v^{\beta}\right)+\beta_{0}\left(M_{0}\left(\mathbf{u}_{1}\right), q\right)=(F, V), \quad \forall V \in V(D) \times L^{2}(D),
\end{array}\right.
$$

where

$$
\left\{\begin{array}{l}
\mathscr{\beta}\left(\mathbf{u}_{1}, \mathbf{u}_{1}, \mathbf{v}\right)=\frac{\delta^{4}}{4}\left(a_{\alpha \beta}\left(u_{1}^{\lambda} \nabla_{\lambda}^{*} u_{1}^{\alpha}-\frac{1}{2} u_{1}^{\alpha} \operatorname{div}^{*} \mathbf{u}_{1}\right), v^{\beta}\right) \\
B_{\beta}\left(\mathbf{u}_{1}, \mathbf{u}_{1}\right)=\frac{\delta^{4}}{4} a_{\alpha \beta}\left(u_{1}^{\lambda} \stackrel{*}{\nabla}_{\lambda} u_{1}^{\alpha}-\frac{1}{2} u_{1}^{\alpha} \operatorname{div}^{*} \mathbf{u}_{1}\right), B^{\alpha}\left(\mathbf{u}_{1}, \mathbf{u}_{1}\right)=a^{\alpha \beta} B_{\beta}\left(\mathbf{u}_{1}, \mathbf{u}_{1}\right), \\
B_{3}\left(\mathbf{u}_{1}, \mathbf{u}_{1}\right)=\frac{\delta^{4}}{4} b_{\lambda \sigma} u_{1}^{\lambda} u_{1}^{\sigma}, \\
\mathscr{O}_{\beta}\left(p_{0}\right)=d_{\beta}^{\alpha} \stackrel{*}{\nabla}_{\alpha} p_{0}-\frac{4 \delta^{3}}{3} \stackrel{\nabla}{\nabla}_{\beta} H p_{0}, \\
(F, V)=\alpha_{0}\left(F_{\beta}^{1}-\frac{\eta_{\beta}^{\alpha}}{4} \frac{\mu \delta^{3}}{3} F_{\alpha}^{2}-\frac{\delta^{3}}{3} \stackrel{*}{*}_{\alpha} p_{1}, v^{\alpha}\right)+\beta_{0}\left(a^{\alpha \beta} \stackrel{\nabla}{\alpha}_{\alpha} F_{\beta}^{2}, q\right) .
\end{array}\right.
$$

Lemma 1 Assume that the metric tensor and curvature tensor of $\mathfrak{I}$ satisfy $a_{\alpha \beta} \in C^{1}(\bar{D})$ and $b_{\alpha \beta} \in C^{1}(\mathbf{D})$ respectively. Then viscosity tensor of $\mathfrak{I} \mu a^{\alpha \beta} a_{\lambda \sigma}$ and metric tensor $a^{\alpha \beta}$ are positive definition, i.e. for any symmetric matrix $\left(t_{\alpha}^{\lambda}\right)$, there exists two constants $\lambda_{*}(D, \mathfrak{I}), \lambda_{0}$ independent of $\left(t_{\alpha}^{\lambda}\right)$ such that

$$
\begin{aligned}
& 2 \mu a^{\alpha \beta} a_{\lambda \sigma} t_{\alpha}^{\lambda} t_{\beta}^{\sigma} \geq \mu \lambda_{*} \sum_{\alpha, \lambda}\left|t_{\alpha}^{\lambda}\right|^{2}, \\
& a^{\alpha \beta} t_{\alpha} t_{\beta} \geq \lambda_{0} \sum_{\alpha}\left|t_{\alpha}\right|^{2}, \quad a_{\alpha \beta} t^{\alpha} t^{\beta} \geq \lambda_{0} \sum_{\alpha}\left|t_{\alpha}\right|^{2},
\end{aligned}
$$


Therefore,

$$
\begin{aligned}
& a_{*}(\mathbf{u}, \mathbf{u}):=\left(a^{\alpha \beta} a_{\lambda \sigma} \stackrel{\nabla}{\nabla}_{\alpha} u^{\lambda}, \stackrel{\nabla}{\beta}_{\beta} u^{\beta}\right) \geq 2 \mu \lambda_{*} \sum_{\alpha, \beta}\left\|\nabla_{\alpha}^{*} u^{\beta}\right\|_{0, D}^{2}:=2 \mu \lambda_{*}\left|\nabla^{*} \mathbf{u}\right|_{1, D}^{2}, \\
& a_{0}(p, p) \geq \frac{\delta^{3}}{3\|\stackrel{\nabla}{\nabla} p\|_{0, D}^{2}} \geq \frac{\delta^{3}}{3 \lambda_{0}|p|_{1, D}^{2}} .
\end{aligned}
$$

Furthermore, If $H, K \in C^{1}(D)$ and the thickness $\delta$ of boundary domain small enough, then bilinear form $\left(Q_{\alpha \beta} u^{\alpha}, v^{\beta}\right)$ is positive

$$
\left|\left(Q_{\alpha \beta} u^{\alpha}, u^{\beta}\right)\right| \geq 2 \mu \delta\|\mathbf{u}\|_{0, D}, \quad \forall \mathbf{u} \in V(D)
$$

Proof The proof of (4.4) can be found in ([1] [4]). It remain to prove (4.6). By virtue of the positive definition of metric tensor $a_{\alpha \beta}$ and assumption of lemma and using Hoelder inequality, we assert that

$$
\begin{aligned}
\left(Q_{\alpha \beta} u_{1}^{\alpha}, u_{1}^{\beta}\right) & =2 \mu\left[\frac{\delta}{8}\left(a_{\alpha \beta} u_{1}^{\alpha}, u_{1}^{\beta}\right)+\left(\left(\left(\frac{3}{2} \frac{H \delta^{2}}{2}+\left(4 K-2 H^{2}\right) \frac{\delta^{3}}{3}\right) a_{\alpha \beta}+\left(\frac{\delta^{2}}{2}-\frac{11 H \delta^{3}}{3}\right) b_{\alpha \beta}\right) u_{1}^{\alpha}, u_{1}^{\beta}\right)\right] \\
& \geq 2 \mu\left[\frac{\delta}{8} \lambda_{0}\left\|\mathbf{u}_{1}\right\|_{0, D}^{2}-\delta^{2} C\left\|\mathbf{u}_{1}\right\|_{0, D}^{2}\right]=2 \mu\left(\frac{\delta}{8}-C \delta^{2}\right)\left\|\mathbf{u}_{1}\right\|_{0, D}^{2} \geq 2 \mu \delta\left\|\mathbf{u}_{1}\right\|_{0, D}^{2} \quad \forall \mathbf{u}_{1} \in V(D), \text { if } C \delta \leq \frac{1}{8},
\end{aligned}
$$

where $C$ is a constant independent of $\mathbf{u}_{1}$ depending $(H, K), \lambda_{0}$. The proof is complete. \#

Lemma 2 Assume that the two-dimensional manifold $\mathfrak{I}$ is smooth enough such that the metric tensor $a_{\alpha \beta}$ of $\mathfrak{I}$ and curvature tensor $b_{\alpha \beta}$ satisfy $a_{\alpha \beta}, b_{\alpha \beta} \in C^{1}(\bar{D})$. Then the bilinear forms $a\left(\mathbf{u}_{1}, \mathbf{v}\right), \mathscr{L}(U, V)$ defined by (4.1): $\mathbf{W}(D):=\mathbf{H}_{p}^{1}(D) \times H^{1}(D) \Rightarrow \Re$ is symmetric, continuous

$$
\left\{\begin{array}{l}
a(\mathbf{u}, \mathbf{v})=a(\mathbf{v}, \mathbf{u}), \quad \mathscr{L}(U, V)=\mathscr{L}(V, U), \quad \forall U, V \in \mathbf{W}(D), \\
|a(\mathbf{u}, \mathbf{v})| \leq \delta C|\mathbf{u}|_{1, D}|\mathbf{v}|_{1, D}, \\
|\mathscr{L}(U, V)| \leq \alpha_{0} 2 \mu \delta C\left|\mathbf{u}_{1}\right|_{1, D}|\mathbf{v}|_{1, D}+\frac{\beta_{0} \delta^{3}}{3} C\left|p_{0}\right|_{1, D}|q|_{1, D}, \quad \forall U, V \in \mathbf{W}(D),
\end{array}\right.
$$

where $H_{p}^{1}(D)=\left\{u \in H^{1}(D),\left.u\right|_{\Gamma}\right.$ satisfying P.B.C. $\}$. Furthermore if $\delta$ is smaller enough such that

$$
\delta \leq \min \left\{\frac{1}{\sqrt{\lambda_{*}}}, \frac{1}{8 C}\right\}
$$

then they are also coercive respectively

$$
\left\{\begin{array}{l}
a\left(\mathbf{u}_{1}, \mathbf{u}_{1}\right) \geq 2 \mu\left(\frac{\delta^{3}}{3} C\left|\mathbf{u}_{1}\right|_{1, D}^{2}+\delta\left\|\mathbf{u}_{1}\right\|_{0, D}^{2}\right), \\
\mathscr{L}(U, U) \geq 2 \mu \alpha_{0}\left[\frac{\delta^{3}}{3} C\left|\mathbf{u}_{1}\right|_{1, D}^{2}+\delta\left\|\mathbf{u}_{1}\right\|_{0, D}^{2}\right]+\frac{\delta^{3}}{3} \beta_{0} \lambda_{0}\left|p_{0}\right|_{1, D}^{2}, \quad \forall U \in \mathbf{W}(D)
\end{array}\right.
$$

where $C$ is a constant independent of $U, V$ having different meaning at different place and

$$
\|U\|^{2}=\alpha_{0}\left|\mathbf{u}_{1}\right|_{1, D}^{2}+\beta_{0}\left|p_{0}\right|_{1, D}^{2}, \quad\|V\|^{2}=\alpha_{0}|\mathbf{v}|_{1, D}^{2}+\beta_{0}|q|_{1, D}^{2}, \quad \forall U=\left(\mathbf{u}_{1}, p_{0}\right), \quad V:=(\mathbf{v}, q)
$$

Proof Indeed it is enough to prove the coerciveness (4.8) since the continuous and symmetric are obvious by Hoelder inequality. Since Lemma 1, 


$$
\begin{aligned}
& a\left(\mathbf{u}_{1}, \mathbf{u}_{1}\right)=\frac{2 \mu \delta^{3}}{3}\left(a^{\alpha \lambda} a^{\beta \sigma} \stackrel{e}{e}_{\lambda \sigma}\left(\mathbf{u}_{1}\right), \stackrel{e}{\alpha \beta}_{\alpha \beta}\left(\mathbf{u}_{1}\right)\right)+\left(Q_{\alpha \beta} u_{1}^{\alpha}, u_{1}^{\beta}\right) \geq \frac{2 \mu \delta^{3}}{3} \lambda_{*} \sum_{\alpha, \beta}\|\|_{\alpha \beta}^{*}\left(\mathbf{u}_{1}\right)\left\|_{0, D}^{2}+2 \mu \delta\right\| \mathbf{u}_{1} \|_{0, D}, \\
& \mathscr{L}(U, U) \geq \alpha_{0}\left[\frac{2 \mu \delta^{3}}{3} \lambda_{*} \sum_{\alpha, \beta}\|\|_{\alpha \beta}^{*}\left(\mathbf{u}_{1}\right)\left\|_{0, D}^{2}+2 \mu \delta\right\| \mathbf{u}_{1} \|_{0, D}^{2}\right]+\frac{\delta^{3}}{3} \beta_{0} \lambda_{0}\left|p_{0}\right|_{1, D}^{2},
\end{aligned}
$$

In view of Korn inequality on Riemann manifold (see [4] Th.1.7.9 )

$$
\left\|\nabla \mathbf{u}_{1}\right\|_{0, D}^{2} \leq C\left(\left\|\mathbf{u}_{1}\right\|_{0, D}^{2}+\sum_{\alpha \beta}\left\|e_{\alpha \beta}^{*}\left(\mathbf{u}_{1}\right)\right\|_{0, D}^{2}\right), \quad \sum_{\alpha \beta}\left\|e_{\alpha \beta}^{*}\left(\mathbf{u}_{1}\right)\right\|_{0, D}^{2} \geq \frac{1}{C}\left\|\nabla \mathbf{u}_{1}\right\|_{0, D}^{2}-\left\|\mathbf{u}_{1}\right\|_{0, D}^{2},
$$

we assert that

$$
\begin{aligned}
& a\left(\mathbf{u}_{1}, \mathbf{u}_{1}\right) \geq \frac{2 \mu \delta^{3}}{3} C\left|\mathbf{u}_{1}\right|_{1, D}^{2}+2 \mu \delta\left(1-\delta^{2} \lambda_{*}\right)\left\|\mathbf{u}_{1}\right\|_{0, D}^{2} \geq \frac{2 \mu \delta^{3}}{3} C\left|\mathbf{u}_{1}\right|_{1, D}^{2}+2 \mu \delta\left\|\mathbf{u}_{1}\right\|_{0, D}^{2}, \\
& \mathscr{L}(U, U) \geq \frac{2 \mu \delta^{3}}{3} \alpha_{0} C\left|\mathbf{u}_{1}\right|_{1, D}^{2}++\alpha_{0} 2 \mu \delta\left\|\mathbf{u}_{1}\right\|_{0, D}^{2}+\frac{\delta^{3}}{3} \beta_{0} \lambda_{0}\left|p_{0}\right|_{1, D}^{2},
\end{aligned}
$$

if $\delta$ satisfies $\delta^{2} \leq\left(\lambda_{*}\right)^{-1}$. To sum up, we conclude our proof. \#

Next we consider variational problem (4.2) corresponding to boundary layer Equation (3.4). Let

$$
\begin{aligned}
& A(U, V)=\mathscr{L}(U, V)+\alpha_{0}\left[\mathscr{R}\left(\mathbf{u}_{1}, \mathbf{u}_{1}, \mathbf{v}\right)+\left(\mathscr{O}_{\beta}\left(p_{0}\right), v^{\beta}\right)\right]+\beta_{0}\left(M_{0}\left(\mathbf{u}_{1}\right), q\right), \\
& \forall U, V \in W(D)=V(D) \times L^{2}(D),
\end{aligned}
$$

Lemma 3 Assume that the manifold $\mathfrak{I}$ satisfies that $a_{\alpha \beta}, b_{\alpha \beta}, H, K \in C^{1}(\bar{D})$ such that there exists a constant $C_{\delta}>0$

$$
\max \left\{\left|a_{\alpha \beta}\right|,\left|a^{\alpha \beta}\right|,\left|b_{\alpha \beta}\right|,|K|,|H|\right\} \leq C_{\delta}
$$

The thickness $\delta$ of the boundary layer is small enough. Then bilinear form $A(\cdot, \cdot): \mathbf{W}(D):=\mathbf{H}_{p}^{1}(D) \times H_{p}^{1}(D) \Rightarrow \mathfrak{R}$ defined by (4.11) is continuous:

$$
\begin{gathered}
A(U, W) \leq C\left[\left(2 \mu \alpha_{0}\left(\frac{\delta^{3}}{3}\left\|\mathbf{u}_{1}\right\|\left\|_{1, D}+\delta\right\| \mathbf{u}_{1} \|_{0, D}\right)+\delta^{2} \beta_{0}\left|p_{0}\right|_{1, D}\right)|\mathrm{v}|_{1, D}+\left(\delta^{2}\left|\mathbf{u}_{1}\right|_{1, D}+\delta\left\|\mathbf{u}_{1}\right\|_{0, D}\right)|q|_{1, D}\right], \\
\forall U, W \in \mathbf{W}(D),
\end{gathered}
$$

where $\|\left.|U|\right|_{1, D}:=\left|u_{1}\right|_{1, D}+\left|p_{0}\right|_{1, D}$ and also satisfies following inequality

$$
\left\{\begin{array}{l}
\mathscr{L}(U, U)+\alpha_{0}\left(\mathcal{O}_{\beta}\left(p_{0}\right), u_{1}^{\beta}\right)+\beta_{0}\left(M_{0}\left(\mathbf{u}_{1}\right), p_{0}\right) \geq \mu \delta^{3}\left|\mathbf{u}_{1}\right|_{1, D}^{2}+\mu \alpha_{0} \delta\left\|\mathbf{u}_{1}\right\|_{0, D}^{2}+\frac{1}{4} \frac{\delta^{3}}{3} \beta_{0} \lambda_{0}\left|p_{0}\right|_{1, D}^{2}, \\
A(U, U) \geq \mu \delta^{3}\left(1-C \delta\left\|\mathbf{u}_{1}\right\|_{0, D}\right)\left|\mathbf{u}_{1}\right|_{1, D}^{2}+\mu \alpha_{0} \delta\left(1-C \delta^{3}\left|\mathbf{u}_{1}\right|_{1, D}\right)\left\|\mathbf{u}_{1}\right\|_{0, D}^{2}+\frac{1}{4} \frac{\delta^{3}}{3} \beta_{0} \lambda_{0}\left|p_{0}\right|_{1, D}^{2},
\end{array}\right.
$$

where $\delta$ is small enough and parameters $\left(\alpha_{0}, \beta_{0}\right)$ satisfy

$$
\begin{aligned}
& \delta \leq \frac{3}{4 C_{\delta}} \min \left\{\frac{\beta_{0}}{\left(\alpha_{0}+3 \mu \beta_{0}\right)}, \frac{\alpha_{0}}{\left(\alpha_{0}+4 \mu \beta_{0}\right)}, \frac{\mu \beta_{0}}{\left(\alpha_{0}+2 \mu \beta_{0}\right)}\right\} \cap \min \left\{\frac{1}{\sqrt{\lambda_{*}}}, \frac{1}{8 C}, \frac{1}{2 C_{\delta}}, \frac{\lambda_{*} \mu}{8 C_{\delta}}, \frac{1}{8 C_{\delta}} \sqrt{\frac{3 \lambda_{*}}{\mu C}}\right\}, \\
& \left(\frac{2}{3} \frac{1}{\mu \lambda_{0}} \leq \beta_{0} \leq \frac{\lambda_{0}}{2 \mu} \alpha_{0}\right) \cap\left(\frac{3}{\lambda_{0}} \leq \alpha_{0}\right),
\end{aligned}
$$

Proof It is easy to verify (4.12) by applying Hoelder inequality and Poincare inequality. It remains to prove (4.13). At the first, we recall that the assumptions of the lemma shows 


$$
\sup _{D, \alpha, \beta}\left\{|H|,|K|,|\nabla H|,\left|a_{\alpha \beta}\right|,\left|b_{\alpha \beta}\right|\right\} \leq C_{\delta}
$$

Taking (4.8) into account, from (4.10) it infers

$$
A(U, U): \geq \mathscr{L}(U, U)-\alpha_{0}\left[\left|\mathscr{B}\left(\mathbf{u}_{1}, \mathbf{u}_{1}, \mathbf{v}\right)\right|-\left|\left(\mathscr{O}_{\beta}\left(p_{0}\right), v^{\beta}\right)\right|\right]-\beta_{0}\left|\left(M_{0}\left(\mathbf{u}_{1}\right), q\right)\right|
$$

(1) Since Lemma 1 and (4.3) we have

$$
\begin{aligned}
& \left(\mathcal{O}_{\beta}^{1}\left(p_{0}\right), u_{1}^{\beta}\right)=\left(d_{\beta}^{\alpha} \stackrel{*}{\nabla}_{\alpha}\left(p_{0}\right)-\frac{4 \delta^{3}}{3} \stackrel{*}{\nabla}_{\beta} H p_{0}, u_{1}^{\beta}\right)=\frac{4 \delta^{3}}{3}\left(H p_{0}, \operatorname{div}^{*} \mathbf{u}_{1}\right)+\left(\left(\frac{3}{2} \frac{\delta^{2}}{2} \delta_{\beta}^{\alpha}+\frac{\delta^{3}}{3}\left(H \delta_{\beta}^{\alpha}-b_{\beta}^{\alpha}\right)\right) \stackrel{*}{\nabla}_{\alpha} p_{0}, u_{1}^{\beta}\right) \\
& \left(M_{1}\left(\mathbf{u}_{1}\right), p_{0}\right)=2 \mu\left(\left(\frac{\delta^{2}}{2}-\frac{4 H \delta^{3}}{3}\right) \operatorname{div} \mathbf{u}_{1}, p_{0}\right)+\frac{4 \mu \delta^{3}}{3}\left(b_{\beta}^{\alpha} \stackrel{*}{\nabla}_{\alpha} u_{1}^{\beta}+3 u_{1}^{\beta} \nabla_{\beta}^{*} H, p_{0}\right),
\end{aligned}
$$

Moreover, using Godazzi formula $\stackrel{*}{\nabla}_{\alpha} b_{\beta \sigma}=\stackrel{*}{\nabla}_{\beta} b_{\alpha \sigma}$, we obtain

$$
\begin{aligned}
b_{\beta}^{\alpha} \stackrel{*}{\nabla}_{\alpha} u_{1}^{\beta}=\stackrel{*}{\nabla}_{\alpha}\left(b_{\beta}^{\alpha} u_{1}^{\beta}\right)-u_{1}^{\beta} \stackrel{*}{\nabla}_{\alpha} b_{\beta}^{\alpha} & =\stackrel{*}{\nabla}_{\alpha}\left(b_{\beta}^{\alpha} u_{1}^{\beta}\right)-2 H u_{1}^{\beta} \stackrel{*}{\nabla}_{\beta} H, \\
\frac{4 \mu \delta^{3}}{3}\left(b_{\beta}^{\alpha} \stackrel{*}{\nabla}_{\alpha} u_{1}^{\beta}+3 u_{1}^{\beta} \stackrel{*}{\nabla}_{\beta} H, p_{0}\right) & =\frac{4 \mu \delta^{3}}{3}\left(\stackrel{*}{\nabla}_{\alpha}\left(b_{\beta}^{\alpha} u_{1}^{\beta}\right)+u_{1}^{\beta} \stackrel{*}{\nabla}_{\beta} H, p_{0}\right) \\
& =-\frac{4 \mu \delta^{3}}{3}\left(b_{\beta}^{\alpha} u_{1}^{\beta}, \stackrel{\nabla}{\nabla}_{\alpha} p_{0}\right)+\frac{4 \mu \delta^{3}}{3}\left(u_{1}^{\beta} \stackrel{*}{\nabla}_{\beta} H, p_{0}\right),
\end{aligned}
$$

Therefore

$$
\begin{aligned}
I= & \alpha_{0}\left(\vartheta_{\beta}^{1}\left(p_{0}\right), u_{1}^{\beta}\right)+\beta_{0}\left(M_{1}\left(\mathbf{u}_{1}\right), p_{0}\right)=\left(\left(\frac{2 \mu \beta_{0} \delta^{2}}{2}+\frac{4 \delta^{3}}{3}\left(\alpha_{0}-2 \mu \beta_{0}\right) H\right) p_{0}, \operatorname{div} \mathbf{u}_{1}\right) \\
& +\left(\left(\frac{3}{2} \frac{\alpha_{0} \delta^{2}}{2} \delta_{\beta}^{\alpha}+\frac{\delta^{3}}{3}\left(\alpha_{0} H \delta_{\beta}^{\alpha}+\left(\alpha_{0}+4 \mu \beta_{0}\right) b_{\beta}^{\alpha}\right)\right) \stackrel{\nabla}{\nabla}_{\alpha} p_{0}, u_{1}^{\beta}\right)+\left(\frac{4 \mu \beta_{0} \delta^{3}}{3} \stackrel{\nabla}{\nabla}_{\beta} H p_{0}, u_{1}^{\beta}\right),
\end{aligned}
$$

Thanks to

$$
\begin{aligned}
& \operatorname{div}_{1}^{*} \mathbf{u}_{1} \partial_{\beta} u_{1}^{\beta}+\partial_{\beta} \ln \sqrt{a} u_{1}^{\beta} \\
& I=\left(\left(\frac{2 \mu \beta_{0} \delta^{2}}{2}+\frac{4 \delta^{3}}{3}\left(\alpha_{0}-2 \mu \beta_{0}\right) H\right) p_{0}, \partial_{\beta} u_{1}^{\beta}\right) \\
&+\left(\left(\frac{3}{2} \frac{\alpha_{0} \delta^{2}}{2} \delta_{\beta}^{\alpha}+\frac{\delta^{3}}{3}\left(\alpha_{0} H \delta_{\beta}^{\alpha}+\left(\alpha_{0}+4 \mu \beta_{0}\right) b_{\beta}^{\alpha}\right)\right) \stackrel{*}{\nabla}_{\alpha} p_{0}, u_{1}^{\beta}\right) \\
&+\left(\left(\left(\frac{2 \mu \beta_{0} \delta^{2}}{2}+\frac{4 \delta^{3}}{3}\left(\alpha_{0}-2 \mu \beta_{0}\right) H\right) \partial_{\beta} \ln \sqrt{a}+\mu \beta_{0} \stackrel{*}{\nabla}_{\beta} H\right) p_{0}, u_{1}^{\beta}\right) \\
&= I_{1}+I_{2}+I_{3},
\end{aligned}
$$

We assert that

$$
\begin{aligned}
& I_{1}=\mu \beta_{0} \delta^{2}\left(\left(1+\frac{4}{3} \delta \frac{\left(\alpha_{0}-2 \mu \beta_{0}\right) H}{\mu \beta_{0}}\right) p_{0}, \partial_{\beta} u_{1}^{\beta}\right) \leq \mu \beta_{0} \delta^{2}\left|1+\frac{4}{3} \delta \frac{\left(\alpha_{0}-2 \mu \beta_{0}\right) H}{\mu \beta_{0}}\right| C\left|p_{0}\right|_{1, D}\left|\mathbf{u}_{1}\right|_{1, D} \\
& \quad \leq 2 C \mu \beta_{0} \delta^{2}\left|p_{0}\right|_{1, D}\left|\mathbf{u}_{1}\right|_{1, D}, \\
& \left|1+\frac{4}{3} \delta \frac{\left(\alpha_{0}-2 \mu \beta_{0}\right) H}{\mu \beta_{0}}\right| \leq\left|1+\frac{4}{3} \delta \frac{\left(\alpha_{0}+2 \mu \beta_{0}\right) C_{\delta}}{\mu \beta_{0}}\right| \leq 2 .
\end{aligned}
$$

Second inequality shows 


$$
\delta \leq \frac{3}{4} \frac{\mu \beta_{0}}{\left(\alpha_{0}+2 \mu \beta_{0}\right) C_{\delta}}
$$

Using Young inequality

$$
a b \leq \frac{1}{4 \varepsilon} b^{2}+\varepsilon a^{2}
$$

we have

$$
I_{1} \leq \frac{1}{4} \frac{\delta^{3}}{3} \beta_{0} \lambda_{0}\left|p_{0}\right|_{1, D}^{2}+12 \frac{\beta_{0}}{\lambda_{0}} C^{2} \mu^{2} \delta\left|u_{1}\right|_{1, D}^{2}
$$

By similar manner,

$$
\begin{aligned}
& I_{2}=\frac{3}{4} \alpha_{0} \delta^{2}\left(\left(\delta_{\beta}^{\alpha}+\frac{4}{9 \alpha_{0}} \delta\left(\alpha_{0} H \delta_{\beta}^{\alpha}+\left(\alpha_{0}+4 \mu \beta_{0}\right) b_{\beta}^{\alpha}\right)\right) \nabla_{\alpha}^{*} p_{0}, u_{1}^{\beta}\right) \\
& \leq \frac{3}{4} \alpha_{0} \delta^{2}\left(1+\frac{4}{9 \alpha_{0}} \delta C_{\delta}\left(\alpha_{0}+4 \mu \beta_{0}\right)\right)\left|p_{0}\right|_{1, D}\left\|\mathbf{u}_{1}\right\|_{0, D} \\
& \leq \alpha_{0} \delta^{2}\left|p_{0}\right|_{1, D}\left\|\mathbf{u}_{1}\right\|_{0, D} \leq \frac{1}{4} \frac{\delta^{3}}{3} \beta_{0} \lambda_{0}\left|p_{0}\right|_{1, D}^{2}+\frac{\alpha_{0}^{2}}{\lambda_{0} \beta_{0}}\left\|\mathbf{u}_{1}\right\|_{0, D}^{2}, \\
& \text { if } \delta \leq \frac{3}{4} \frac{\alpha_{0}}{C_{\delta}\left(\alpha_{0}+4 \mu \beta_{0}\right)}, \\
& \left\{\begin{aligned}
I_{3} & =\left(\left(\frac{2 \mu \beta_{0} \delta^{2}}{2}+\frac{4 \delta^{3}}{3}\left(\left(\alpha_{0}-2 \mu \beta_{0}\right) H \partial_{\beta} \ln \sqrt{a}+\mu \beta_{0} \stackrel{*}{\nabla}_{\beta} H\right)\right) p_{0}, u_{1}^{\beta}\right) \\
& =\mu \beta_{0} \delta^{2}\left(1+\frac{4}{3 \beta_{0}} \delta\left(\left(\alpha_{0}-2 \mu \beta_{0}\right) H \partial_{\beta} \ln \sqrt{a}+\mu \beta_{0} \stackrel{\nabla}{\nabla}_{\beta} H\right) p_{0}, u_{1}^{\beta}\right) \\
& \leq \mu \beta_{0} \delta^{2}\left(1+\frac{4}{3} \frac{\delta}{\beta_{0}} C_{\delta}\left(\alpha_{0}+3 \mu \beta_{0}\right)\right)\left|p_{0}\right|_{1, D}\left\|\mathbf{u}_{1}\right\|_{0, D} \leq 2 \mu \beta_{0} \delta^{2}\left|p_{0}\right|_{1, D}\left\|\mathbf{u}_{1}\right\|_{0, D} \\
& \leq \frac{1}{4} \frac{\delta^{3}}{3} \beta_{0} \lambda_{0}\left|p_{0}\right|_{1, D}^{2}+4 \mu \frac{\beta_{0}}{\lambda_{0}}\left\|u_{1}\right\|_{0, D}^{2}, \\
\text { if } \quad \delta & \leq \frac{3}{4} \frac{\beta_{0}}{C_{\delta}\left(\alpha_{0}+3 \mu \beta_{0}\right)},
\end{aligned}\right.
\end{aligned}
$$

Substituting (4.18-4.20) into (4.16) leads to

$$
\left\{\begin{array}{l}
I \leq \frac{3}{4} \frac{\delta^{3}}{3} \beta_{0} \lambda_{0}\left|p_{0}\right|_{1, D}^{2}+12 \frac{\beta_{0}}{\lambda_{0}} C^{2} \mu^{2} \delta\left|u_{1}\right|_{1, D}^{2}+\delta \frac{\alpha_{0}^{2}+\mu \beta_{0}^{2}}{\lambda_{0} \beta_{0}}\left\|\mathbf{u}_{1}\right\|_{0, D}^{2}, \\
\text { if } \delta \leq \frac{3}{4 C_{\delta}} \min \left\{\frac{\beta_{0}}{\left(\alpha_{0}+3 \mu \beta_{0}\right)}, \frac{\alpha_{0}}{\left(\alpha_{0}+4 \mu \beta_{0}\right)}, \frac{\mu \beta_{0}}{\left(\alpha_{0}+2 \mu \beta_{0}\right)}\right\},
\end{array}\right.
$$

Taking (4.9) into account, it yield

$$
\left\{\begin{array}{l}
\mathscr{L}(U, U)+I \geq 2 \mu \delta\left(\delta^{2}-\frac{\beta_{0}}{\lambda_{0} \alpha_{0}} C \mu\right)\left|\mathbf{u}_{1}\right|_{1, D}^{2}+2 \mu \alpha_{0} \delta\left(1-\frac{\alpha_{0}^{2}+\mu \beta_{0}^{2}}{2 \mu \lambda_{0} \beta_{0} \alpha_{0}}\right)\left\|\mathbf{u}_{1}\right\|_{0, D}^{2}+\frac{1}{4} \frac{\delta^{3}}{3} \beta_{0} \lambda_{0}\left|p_{0}\right|_{1, D}^{2}, \\
\text { if } \delta \leq \frac{3}{4 C_{\delta}} \min \left\{\frac{\beta_{0}}{\left(\alpha_{0}+3 \mu \beta_{0}\right)}, \frac{\alpha_{0}}{\left(\alpha_{0}+4 \mu \beta_{0}\right)}, \frac{\mu \beta_{0}}{\left(\alpha_{0}+2 \mu \beta_{0}\right)}\right\}, \quad \delta \leq \min \left\{\frac{1}{\sqrt{\lambda_{*}}}, \frac{1}{8 C}\right\},
\end{array}\right.
$$

If 


$$
\frac{C \mu \beta_{0}}{\lambda_{0} \alpha_{0}} \leq \frac{\delta^{2}}{2}, \quad \frac{\alpha_{0}+\mu \beta_{0}}{2 \mu \lambda_{0} \alpha_{0} \beta_{0}} \leq \frac{1}{2}
$$

Then

$$
\left\{\begin{array}{l}
\mathscr{L}(U, U)+I \geq \mu \delta^{3}\left|\mathbf{u}_{1}\right|_{1, D}^{2}+\mu \alpha_{0} \delta\left\|\mathbf{u}_{1}\right\|_{0, D}^{2}+\frac{1}{4} \frac{\delta^{3}}{3} \beta_{0} \lambda_{0}\left|p_{0}\right|_{1, D}^{2}, \\
\text { if } \delta \leq \frac{3}{4 C_{\delta}} \min \left\{\frac{\beta_{0}}{\left(\alpha_{0}+3 \mu \beta_{0}\right)}, \frac{\alpha_{0}}{\left(\alpha_{0}+4 \mu \beta_{0}\right)}, \frac{\mu \beta_{0}}{\left(\alpha_{0}+2 \mu \beta_{0}\right)}\right\}, \quad \delta \leq \min \left\{\frac{1}{\sqrt{\lambda_{*}}}, \frac{1}{8 C}\right\},
\end{array}\right.
$$

It is easy to verify that (4.23) is satisfied if the parameters $\left(\alpha_{0}, \beta_{0}\right)$ in the definition (4.1) are held

$$
\frac{2}{3} \frac{1}{\mu \lambda_{0}} \leq \beta_{0} \leq \frac{\lambda_{0}}{2 \mu} \alpha_{0}, \quad \frac{3}{\lambda_{0}} \leq \alpha_{0}
$$

Next we consider trilinear form. Taking into account of

$$
\stackrel{*}{\nabla}_{\lambda} u_{1}^{\alpha}=\partial_{\lambda} u_{1}^{\alpha}+\Gamma^{*}{ }_{\lambda \sigma} u^{\sigma}, \quad \operatorname{div}^{*} \mathbf{u}_{1}=\partial_{\alpha} u_{1}^{\alpha}+\partial_{\alpha} \ln \sqrt{a} u_{1}^{\alpha}
$$

we claim that

$$
\begin{aligned}
& \left|\mathscr{\beta}\left(\mathbf{u}_{1}, \mathbf{u}_{1}, \mathbf{u}_{1}\right)\right|=\frac{\delta^{4}}{4}\left|\left(\stackrel{*}{\nabla}_{\lambda} u_{1}^{\alpha} u_{1}^{\lambda}-u_{1}^{\alpha} \operatorname{div} \mathbf{u}_{1}, a_{\alpha \beta} u_{1}^{\beta}\right)\right| \\
& \leq \frac{\delta^{4}}{4}\left[\left(u_{1}^{\lambda} \partial_{\lambda} u^{\alpha}-u_{1}^{\alpha} \partial_{\lambda} u_{1}^{\lambda}, a_{\alpha \beta} u_{1}^{\beta}\right)+\left(\left(\stackrel{*}{\Gamma}^{\alpha} \lambda \sigma-\partial_{\lambda} \ln \sqrt{a} \delta_{\sigma}^{\alpha}\right) u_{1}^{\sigma} u_{1}^{\lambda}, a_{\alpha \beta} u_{1}^{\beta}\right)\right] \leq \frac{\delta^{4}}{4} C\left(\left|\mathbf{u}_{1}\right|_{1, D}+\left\|\mathbf{u}_{1}\right\|_{0, D}\right)\left\|\mathbf{u}_{1}\right\|_{0,4, D}^{2},
\end{aligned}
$$

By Ladyzhenskya inequality (Temam [11])

$$
\left\|\mathbf{u}_{1}\right\|_{0,4, D}^{4} \leq 2\left\|\mathbf{u}_{1}\right\|_{0, D}^{2}\left\|\nabla \mathbf{u}_{1}\right\|_{0, D}^{2}=2\left\|\mathbf{u}_{1}\right\|_{0, D}^{2}\left|\mathbf{u}_{1}\right|_{1, D}^{2}
$$

it infers that

$$
\left|\mathscr{\beta}\left(\mathbf{u}_{1}, \mathbf{u}_{1}, \mathbf{u}_{1}\right)\right| \leq \frac{C \delta^{4}}{4}\left(\left.\left|\mathbf{u}_{1}\right|_{1, D}^{2}\left\|\mathbf{u}_{1}\right\|_{0, D}\left|+\left\|\mathbf{u}_{1}\right\|_{0, D}^{2}\right| \mathbf{u}_{1}\right|_{1, D}\right)
$$

Combing (4.15) (4.24) and (4.27), we obtain

$$
A(U, U) \geq \mu \delta^{3}\left(1-C \delta\left\|\mathbf{u}_{1}\right\|_{0, D}\right)\left|\mathbf{u}_{1}\right|_{1, D}^{2}+\mu \alpha_{0} \delta\left(1-C \delta^{3}\left|\mathbf{u}_{1}\right|_{1, D}\right)\left\|\mathbf{u}_{1}\right\|_{0, D}^{2}+\frac{1}{4} \frac{\delta^{3}}{3} \beta_{0} \lambda_{0}\left|p_{0}\right|_{1, D}^{2}
$$

This complete our proof. \#

Theorem 3 Assume that the hypotheses in Lemma 3 are satisfied and the mapping

$$
\mathbf{u}_{1} \rightarrow \mathscr{B}\left(\mathbf{u}_{1}, \mathbf{u}_{1}, \mathbf{v}\right) \quad \forall \mathbf{v} \in V(D)
$$

is sequentially weakly continuous in $V(D)$

$$
\text { weak } \operatorname{limit}_{m \rightarrow \infty} \mathbf{u}_{1 m}=\mathbf{u}_{*} \text { in } V(D) \text { implies } \operatorname{limit}_{m \rightarrow \infty} B\left(\mathbf{u}_{1 m}, \mathbf{u}_{1 m}, \mathbf{v}\right)=B\left(\mathbf{u}_{*}, \mathbf{u}_{*}, \mathbf{v}\right), \quad \forall \mathbf{v} \in V(D)
$$

Then there exists at least one solution $U=\left(\mathbf{u}_{1}, p_{0}\right)$ of (4.2) satisfying

$$
\begin{aligned}
& \left|\mathbf{u}_{1}\right|_{1, D}+\left\|p_{0}\right\|_{1, D} \leq \rho, \rho:=\frac{\|\mathbf{F}\|_{*}}{M}, \\
& M=\max \left\{\frac{1}{2} \mu \delta^{3}, \frac{1}{2} \mu \alpha_{0} \delta, \frac{1}{4} \frac{\beta_{0} \lambda_{0} \delta^{3}}{3}\right\},
\end{aligned}
$$

where $\delta$ is the thickness of boundary layer, $C_{k}, \lambda, \lambda_{8}$ are constants defined in the followings. 
Proof We begin with constructing a sequence of approximate solutions by Galerkin's method. Since the space $\mathbf{Y}(D)=\mathbf{W}(D)$ is a separable Hilbert space, there exist sequence $\left(\Phi_{i}, i \geq 1\right)$ in $\mathbf{Y}(D)$ such that: 1) for all $i \geq 1$, the elements $\Phi_{1}, \Phi_{2}, \cdots$ are linearly independent; 2) the finite linear combinations of the $\Phi_{i}$ are dense in $\mathbf{Y}(D)$. Such sequence $\left(\Phi_{i}, i \geq 1\right)$ are called a basis of the separable space. Denote by $\mathbf{Y}_{m}(D)$ the subspace of $\mathbf{Y}(D)$ spanned by finite sequence $\Phi_{1}, \Phi_{2}, \cdots, \Phi_{m}$. Then we solve an approximate problem of (4.2)

$$
\left\{\begin{array}{l}
\text { Find } U_{m}:=\left(\mathbf{u}_{1 m}, p_{0 m}\right) \in \mathbf{Y}_{m}(D) \text { such that } \\
A\left(U_{m}, W_{m}\right)=(\mathbf{F}, W), \quad \forall W=(\mathbf{v}, q) \in \mathbf{Y}_{m}(D),
\end{array}\right.
$$

Setting

$$
U_{m}=\sum_{i=1}^{m} C_{i} \Phi_{i}
$$

Problem (4.30) is equivalent to a system of nonlinear equations with m unknowns $C_{i}$. For each $m$ problem (4.30) has at least one solution. In fact, when defining a mapping $/ / / m: \mathbf{Y}_{m}(D) \rightarrow \mathbf{Y}_{m}(D)$ by

$$
\left(\mathscr{C l}_{m}\left(U_{m}\right), \Phi_{i}\right)=A\left(U_{m}, \Phi_{i}\right)-\left(\mathbf{F}, \Phi_{i}\right), \quad 1 \leq i \leq m
$$

where (,$\cdot)$ is the scalar product in $\mathbf{Y}(D), U_{m} \in \mathbf{Y}_{m}(D)$ is a solution of problem (4.30) if only if $\mathscr{N}_{m}\left(U_{m}\right)=0$ Since

$$
(. / / m(U), U)=A(U, U)-(\mathbf{F}, U), \quad \forall U \in \mathbf{Y}_{m}(D)
$$

it follows from (4.28)

$$
\left(/_{m}(U), U\right) \geq \mu \delta^{3}\left(1-C \delta\left\|\mathbf{u}_{1}\right\|_{0, D}\right)\left|\mathbf{u}_{1}\right|_{1, D}^{2}+\mu \alpha_{0} \delta\left(1-C \delta^{3}\left|\mathbf{u}_{1}\right|_{1, D}\right)\left\|\mathbf{u}_{1}\right\|_{0, D}^{2}+\frac{1}{4} \frac{\delta^{3}}{3} \beta_{0} \lambda_{0}\left|p_{0}\right|_{1, D}^{2}-(F, W)
$$

Let $\left|\mathbf{u}_{1}\right|_{1, D}^{2} \leq \rho,\left\|\mathbf{u}_{1}\right\|_{0, D}^{2} \leq \rho,\left|p_{0}\right|_{1, D}^{2} \leq \rho,\left\|p_{0}\right\|_{0, D}^{2} \leq \rho$ Furthermore, assume that

$$
1-C \delta \rho \geq \frac{1}{2}, 1-C \delta^{3} \rho \geq \frac{1}{2} \text {, i.e. } \delta \leq\left(\frac{1}{2 C \rho}\right)^{1 / 3}
$$

if $\delta$ is small enough. Then

if

$$
\begin{aligned}
& (. / /(U), U) \geq \frac{1}{2} \mu \delta^{3}\left|\mathbf{u}_{1}\right|_{1, D}^{2}+\frac{1}{2} \mu \alpha_{0} \delta\left\|\mathbf{u}_{1}\right\|_{0, D}^{2}+\frac{1}{4} \frac{\beta_{0} \lambda_{0} \delta^{3}}{3}\left|p_{0}\right|_{1, D}^{2}-\|\mathbf{F}\|_{*}\|U\|_{1, D} \\
& \geq M\|U\|_{1, D}^{2}-\|\mathbf{F}\|_{*}\|U\|_{1, D}=M\|U\|_{1, D}\left(\|U\|_{1, D}-\|\mathbf{F}\|_{*} / M\right) \geq 0,
\end{aligned}
$$

$$
\left\{\begin{array}{l}
\|U\|_{1, D} \leq \rho, \\
\rho:=\frac{\|\mathbf{F}\|_{*}}{M}, \quad M=\max \left\{\frac{1}{2} \mu \delta^{3}, \frac{1}{2} \mu \alpha_{0} \delta, \frac{1}{4} \frac{\beta_{0} \lambda_{0} \delta^{3}}{3}\right\},
\end{array}\right.
$$

Hence, we conclude

$$
\text { If }\|U\|_{1, D} \leq \rho \text {, Then }\left(. / /_{m}(U), U\right) \geq 0
$$

Moreover, $/ /$ is continuous in a finite dimension space $\mathbf{Y}_{m}$, we can apply following lemma ([12]).

Lemma 4 Let $H$ be a finite dimensional Hilbert space whose scalar product is denoted by (;:) and the corresponding norm by $\mid \cdot$. Let $\Psi$ be a continuous mapping from $H$ into $H$ with the following property: there exists $\zeta>0$ such that

$$
(\Psi(f), f) \geq 0 \quad \forall f \in H \text { with }|f|=\zeta
$$


Then, there exists an element $f$ in $H$ such that

$$
\Psi(f)=0, \quad|f| \leq \zeta
$$

Therefore there exists a solution $U_{m}$ for problem (4.30) with

$$
\left\|U_{m}\right\|_{1, D} \leq \rho
$$

This shows that the sequence ( $U_{m}$ of the solutions to (4.30) in $\mathbf{Y}_{m}$ are uniformly bounded. Therefore we can extract a subsequence (still denoted by $U_{m}$ ) such that

$$
U_{m} \rightarrow \text { (weakly) } U_{*} \text { in } \mathbf{Y}(D) \text { as } m \rightarrow \infty
$$

Then, the compactness of the embedding of $\mathbf{Y}(D)$ into $L^{2}(D)^{3}$ implies that

$$
U_{m} \rightarrow \text { (strongly) } U_{*} \text { in } L^{2}(D)^{3} \text { as } m \rightarrow \infty
$$

Since $\mathbf{Y}_{m}$ is dense in $\mathbf{Y}(D)$, it is obvious that if

$$
B\left(u_{*}, u_{*}, v\right)=\operatorname{limit}_{m \rightarrow \infty} B\left(\mathbf{u}_{1 m}, \mathbf{u}_{1 m}, v\right)
$$

Taking the limit of both sides of (4.30) implies

$$
A\left(U_{*}, W\right)=(\mathbf{F}, W), \quad \forall W \in \mathbf{Y}_{m}(D)
$$

therefore

$$
A\left(U_{*}, W\right)=(\mathbf{F}, W), \quad \forall W \in \mathbf{Y}(D)
$$

Then $U_{*}$ is a solution of (4.2) and which satisfies

$$
\left\|U_{*}\right\|_{1, D} \leq \rho
$$

The proof is complete. \#

Remark The mapping $\mathbf{u}_{1} \rightarrow \mathscr{\beta}\left(\mathbf{u}_{1}, \mathbf{u}_{1}, \mathbf{v}\right) \forall \mathbf{v} \in V(D)$ is sequentially weakly continuous in $V(D)$ can be found in [3].

\section{Dimensional Split Method for Exterior Flow Problem around an Obstacle and a Two Scale Parallel Algorithms}

In this section, we proposal a dimensional split algorithm for the three dimensional exterior flow around a obstacle occupied by $\hat{\Omega} \subset \mathfrak{R}^{3}$. $\mathfrak{I}=\partial \hat{\Omega}$ is a smooth surface of the obstacle and $\mathfrak{R}^{3}=\Omega \cup \hat{\Omega}$. Assume that $\Omega$ is decomposed by a series of geometric parallel surfaces $\mathfrak{I}_{i}, i=1,2, \cdots$ into a series of stream layer $\Omega_{i, i+1}$ bounded by $\mathfrak{I}_{i}, \mathfrak{I}_{i+1}$ such that $\partial \Omega_{i, i+1}=\mathfrak{I}_{i} \cup \mathfrak{I}_{i+1}$.

On every surface $\mathfrak{I}_{k}, k=0,1,2, \cdots N$, it generalis a global system including one system of BLE I on the boundary surface $\mathfrak{I}$ of the obstacle and $N-1$ systems of flexible boundary equations BLE II on $\mathfrak{I}_{1}, \mathfrak{I}_{2}, \cdots, \mathfrak{I}_{N-1}$ :

$$
\begin{aligned}
& \operatorname{BLEI}\left(u_{1}^{\alpha}, p_{0}\right)=\mathbf{F}^{0}, \\
& \operatorname{SLEII}\left(u_{0}^{\alpha}, p_{0}\right)=\mathbf{F}^{1}, \cdots, \operatorname{SLEII}\left(u_{0}^{\alpha}, p_{0}\right)=\mathbf{F}^{N-1},
\end{aligned}
$$

where right terms are given by

$$
\left\{\begin{array}{l}
F_{\beta}^{1}=\delta a_{\alpha \beta} h_{t}^{\alpha}, F_{3}^{1}=\delta h_{t}^{3}, F_{\beta}^{2}=\delta^{2} a_{\alpha \beta} h_{t}^{\alpha}, F_{3}^{1}=\delta^{2} h_{t}^{3}, F_{\beta}^{0}=a_{\alpha \beta}\left(h_{t}^{\alpha}-h_{b}^{\alpha}\right), F_{3}^{0}=h_{t}^{3}-h_{b}^{3}, \\
h_{b}^{\alpha}=\left.\mu u_{1}^{\alpha}\right|_{\mathfrak{I}}, u_{b}^{3}=-\left.p_{0}\right|_{\mathfrak{I}}, \\
h_{t}^{\alpha}=\left.\mu\left(u_{1}^{\alpha}+a^{\alpha \beta} \nabla_{\beta} u_{0}^{3}-b_{\beta}^{\alpha} u_{0}^{\beta}\right)\right|_{\mathfrak{I}(\delta)}, \\
h_{t}^{3}=-p_{0}+\left.2 \mu u_{1}^{3}\right|_{\mathfrak{I}(\delta)},
\end{array}\right.
$$


The features of these systems are that the right terms of them depend upon the solution of next system, for example, the right term of $k$ th-system depend upon the solution of $(k+1)$ th. system. It is better to apply alterative iteration algorithm to solve these systems. That is

(1) Suppose that right hands $\mathbf{F}^{0}, \mathbf{F}^{1}, \mathbf{F}^{2}, \cdots$, are known;

(2) Solve system of BLEI, BLEII $(k), k=1,2, \cdots, N-1$

(3) Modifying $\mathbf{F}^{0}, \mathbf{F}^{1}, \mathbf{F}^{2}$ by using results obtained, then goto (2) to be continuous until reach certainly accuracy.

In order to find solution of Navier-Stokes equations at any point $\mathrm{P}$ in Exterior domain $\Omega \in \mathfrak{R}^{3}$

(i) Identify point $P$ in which stream layer $\Omega_{i, i+1}$ bounded by $\mathfrak{I}_{i} \cup \mathfrak{I}_{i+1}$, then set $P=\left(x^{\alpha}, \xi\right)$ in local coordinate system;

(ii) $\mathbf{u}(P)=\mathbf{u}_{0}(x)+\mathbf{u}_{1}(x) \xi+\mathbf{u}_{2}(x) \xi^{2}, p(P)=p_{0}(x)+p_{1}(x) \xi+p_{2}(x) \xi^{2}$

where $\left(\mathbf{u}_{0}(x), \mathbf{u}_{1}(x), \mathbf{u}_{2}(x), p_{0}(x) p_{1}(x), p_{2}(x)\right)$ are solution of BLE on $\mathfrak{I}_{i}$.

In details,

(I) For $i=0$ i.e. solid surface with non-slip boundary condition, we give the boundary layer equations BLE I (3.2) on the boundary surface $\mathfrak{I}=\mathfrak{I}_{0}$ of obstacle. from Theorem 2 , three unknown $\left(u_{1}^{\alpha}, p_{0}\right)$ solve

$$
\begin{aligned}
& {\left[-\frac{\mu \delta^{3}}{3}\left[\stackrel{*}{\Delta} u_{1}^{\alpha}+a^{\alpha \lambda} \stackrel{*}{\nabla}_{\lambda} \operatorname{div} \mathbf{u}_{1}+K u_{1}^{\alpha}\right]+a^{\alpha \beta} Q_{\beta \sigma} u_{1}^{\sigma}+\frac{\delta^{3}}{3} a^{\alpha \beta} \stackrel{*}{\nabla}_{\beta} p_{1}\right.} \\
& +\frac{\delta^{4}}{4}\left(u_{1}^{\lambda} \nabla_{\lambda}^{*} u_{1}^{\alpha}-u_{1}^{\alpha} \operatorname{div} \mathbf{u}_{1}\right)+d^{\alpha \beta} \stackrel{*}{\nabla}_{\beta} p_{0}-\frac{4 \delta^{3}}{3} a^{\alpha \beta} \stackrel{*}{\nabla}_{\beta}\left(H p_{0}\right)=a^{\alpha \beta} \mathscr{F}_{\beta}^{1}, \\
& -\frac{\delta^{3}}{3} \stackrel{*}{\Delta} p_{0}+M_{0}\left(\mathbf{u}_{1}\right)=a^{\alpha \beta} \stackrel{*}{\nabla}_{\beta} F_{\alpha}^{2} \text {, } \\
& \left.\left(\mathbf{u}_{1}, p_{0}\right)\right|_{\partial D} \text { satisfies periodic boundary condition }
\end{aligned}
$$

and six unknown $\left(u_{1}^{3}, \mathbf{u}_{2}, p_{1}, p_{2}\right)$ can be found by six algebraic equations

$$
\left\{\begin{array}{l}
u_{1}^{3}=0, \frac{2 \mu \delta^{3}}{3} u_{2}^{\alpha}=a^{\alpha \beta} F_{\beta}^{2}-a^{\alpha \beta} \zeta_{\beta \sigma} u_{1}^{\sigma}-\frac{\delta^{3}}{3} a^{\alpha \beta} \stackrel{\nabla}{\beta}_{\beta} p_{0}, \\
2 u_{2}^{3}+\operatorname{div}\left(\mathbf{u}_{1}\right)=0, \\
\frac{\delta^{3}}{3} p_{2}+\left(\frac{\delta^{2}}{2}-\frac{4 H \delta^{3}}{3}\right) p_{1}+\kappa_{1} p_{0}-\frac{\delta^{4}}{4} b_{\alpha \beta} u_{1}^{\alpha} u_{1}^{\beta}+\frac{2 \mu \delta^{3}}{3} \beta_{0}\left(\mathbf{u}_{1}\right)=-F_{3}^{1}, \\
\frac{2 \delta^{3}}{3} p_{1}+2\left(\frac{\delta^{2}}{2}-\frac{3 H \delta^{3}}{3}\right) p_{0}-\frac{5 \mu \delta^{3}}{3} \operatorname{div} \mathbf{u}_{1}=F_{3}^{2},
\end{array}\right.
$$

Associated variational formulations with (5.1) is given by

$$
\left\{\begin{array}{l}
\text { Find }\left(\mathbf{u}_{1}, p_{0}\right) \in V(D) \times M(D) \text { such that } \\
A_{1}\left(\left(\mathbf{u}_{1}, p_{0}\right),\left(\mathbf{v}_{1}, q\right)\right)+b\left(\mathbf{u}_{1}, \mathbf{u}_{1}, \mathbf{v}_{1}\right)+\frac{\alpha_{0} \delta^{3}}{3}\left(\left(H p_{0}-p_{1}\right), \operatorname{div}\left(\mathbf{v}_{1}\right)\right) \\
\quad-\alpha_{0}\left(p_{0}, \stackrel{\nabla}{\nabla}_{\alpha}\left(d_{\beta}^{\alpha} v_{1}^{\beta}\right)\right)+\beta_{0}\left(M_{0}\left(\mathbf{u}_{1}\right), q\right)=\alpha_{0}\left(\mathscr{F}_{\beta}^{1} v^{\beta}\right), \quad \forall\left(\mathbf{v}_{1}, q\right) \in V(D) \times M(D),
\end{array}\right.
$$

where $\alpha_{0}, \beta_{0}$ are two positive arbitrary constants, the bilinear forms and trilinear form are

$$
\left\{\begin{array}{l}
A_{1}\left(\left(\mathbf{u}, p_{0}\right),(\mathbf{v}, q)\right):=\frac{2 \mu \alpha_{0} \delta^{3}}{3}\left(a^{\alpha \beta} a^{\lambda \sigma} \stackrel{*}{e}_{\alpha \lambda}(\mathbf{u}), \stackrel{*}{e}_{\beta \sigma}(\mathbf{v})\right)+\alpha_{0}\left(Q_{\alpha \beta} u_{1}^{\alpha}, v_{1}^{\beta}\right)+\frac{\varepsilon \delta^{3}}{3} \beta_{0}\left(\stackrel{*}{\nabla} p_{0}, \stackrel{*}{\nabla} q\right), \\
b\left(\mathbf{u}_{1}, \mathbf{u}_{1}, \mathbf{v}_{1}\right)=\frac{\alpha_{0} \delta^{4}}{4}\left(u_{1}^{\lambda} \stackrel{*}{\nabla}_{\lambda} u_{1}^{\alpha}-u_{1}^{\alpha} \operatorname{div} \mathbf{u}_{1}, a_{\alpha \beta} v_{1}^{\beta}\right),
\end{array}\right.
$$

and 


$$
\left\{\begin{array}{l}
\zeta_{\alpha \beta}=2 \mu\left[\left(\frac{\delta^{2}}{2}-\frac{2 H \delta^{3}}{3}\right) a_{\alpha \beta}+\frac{2 \delta^{3}}{3} b_{\alpha \beta}\right], \\
Q_{\alpha \beta}=2 \mu\left[\left(\frac{1}{8} \delta+\frac{3}{2} \frac{H \delta^{2}}{3}+\frac{\left(4 K-2 H^{2}\right) \delta^{3}}{3}\right) a_{\alpha \beta}+\left(\frac{\delta^{2}}{2}-\frac{11 H \delta^{3}}{3}\right) b_{\alpha \beta}\right], \\
d^{\alpha \beta}=\left(\frac{3}{2} \frac{\delta^{2}}{2}+\frac{H \delta^{3}}{3}\right) a^{\alpha \beta}-\frac{\delta^{3}}{3} b^{\alpha \beta}, \\
M_{0}\left(u_{1}\right)=2 \mu\left(\frac{\delta^{2}}{2}-\frac{4 H \delta^{3}}{3}\right) \operatorname{div}_{1}^{*}+\frac{4 \mu \delta^{3}}{3} b_{\beta}^{\alpha} \stackrel{\nabla}{\nabla}_{\alpha} u_{1}^{\beta}+\frac{12 \mu \delta^{3}}{3} u_{1}^{\alpha} \stackrel{\nabla}{\nabla}_{\alpha}^{*} H, \\
F_{p}=a^{\alpha \beta} \stackrel{\nabla}{\alpha}_{\alpha} F_{\beta}^{2}, \\
a^{\alpha \beta} \cdot F_{\beta}^{1}=a^{\alpha \beta} F_{\beta}^{1}-\left[\frac{3}{4} \delta^{-1} a^{\alpha \beta}-\left(H a^{\alpha \beta}+b^{\alpha \beta}\right)\right] F_{\beta}^{2}+\frac{\mu}{2} \frac{\delta^{3}}{3} a^{\alpha \beta} \stackrel{\nabla}{\nabla}_{\beta} u_{2}^{3},
\end{array}\right.
$$

The right terms are given by

$$
\left\{\begin{array}{l}
a^{\alpha \beta} F_{\beta}^{1}=\delta h_{t}^{\alpha}, \quad F_{3}^{1}=\delta h_{t}^{3}, \quad a^{\alpha \beta} F_{\beta}^{2}=\delta^{2} h_{t}^{\alpha}, \quad F_{3}^{2}=\delta^{2} h_{t}^{3}, \\
h_{t}^{\alpha}=\left.\mu\left(u_{1}^{\alpha}+a^{\alpha \beta} \stackrel{*}{\nabla}_{\beta} u_{0}^{3}-b_{\beta}^{\alpha} u_{0}^{\beta}\right)\right|_{\mathfrak{I}(\delta)}, \quad h_{t}^{3}=\left.\left(-p_{0}+2 \mu u_{1}^{3}\right)\right|_{\mathfrak{I}(\delta)},
\end{array}\right.
$$

where $\mathfrak{I}(\delta)=\mathfrak{I}_{1}$.

(II) For $i=1,2, \cdots, N-1$, i.e. on flexible surfaces, corresponding boundary layer equations SLE II (for $\mathbf{u}_{0}, \mathbf{u}_{1}, p_{0}$ ) at flexible surface (artificial interface) $\mathfrak{I}_{i}, \quad i=1,2, \cdots, N-1$ are given by (3.8) and (3.9)

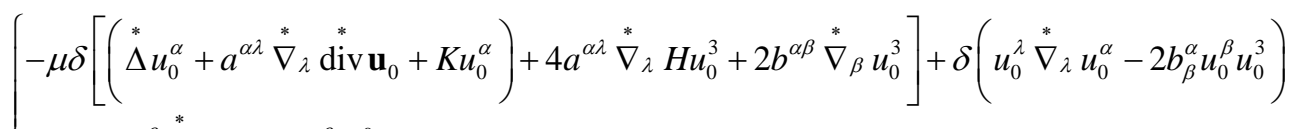

$$
\begin{aligned}
& -\delta a^{\alpha \beta} \stackrel{*}{\nabla}_{\beta} p_{0}=a^{\alpha \beta} F_{\alpha}^{0}, \\
& -2 \mu \delta \stackrel{*}{\Delta} u_{0}^{3}-2 \mu \delta \beta_{0}\left(\mathbf{u}_{0}\right)+\delta\left[u_{0}^{\lambda} \stackrel{*}{\nabla}_{\lambda} u_{0}^{3}+b_{\lambda \sigma} u_{0}^{\lambda} u_{0}^{\sigma}\right]-2 H \delta p_{0}-\mu \delta \operatorname{div}^{*} \mathbf{u}_{1}=F_{3}^{0}, \\
& \mu \delta u_{1}^{\alpha}=a^{\alpha \beta} F_{\beta}^{1}, \quad-\delta p_{0}=F_{3}^{1}, \quad u_{1}^{3}+\gamma_{0}\left(\mathbf{u}_{0}\right)=0 \\
& F_{\beta}^{0}=a_{\alpha \beta}\left(h_{t}^{\alpha}-h_{b}^{\alpha}\right), \quad F_{3}^{0}=h_{t}^{3}-h_{b}^{3}, \quad F_{\beta}^{1}=a_{\alpha \beta} h_{t}^{\alpha} \delta, \quad F_{3}^{1}=\delta h_{t}^{3}, \\
& h_{b}^{\alpha}=\mu u_{1}^{\alpha}(k), \quad h_{b}^{3}=-p_{0}(k), \quad h_{t}^{3}=\frac{1}{2}\left[\left(-p_{0}+2 \mu u_{1}^{3}\right)(k+1)+\left(-p_{0}+2 \mu u_{1}^{3}\right)(k-1)\right], \\
& h_{t}^{\alpha}=\frac{1}{2} \mu\left[\left(u_{1}^{\alpha}+a^{\alpha \beta} \nabla_{\beta}^{*} u_{0}^{3}-b_{\beta}^{\alpha} u_{0}^{\beta}\right)(k+1)+\left(u_{1}^{\alpha}+a^{\alpha \beta} \stackrel{*}{\nabla}_{\beta} u_{0}^{3}-b_{\beta}^{\alpha} u_{0}^{\beta}\right)(k-1)\right] \text {, }
\end{aligned}
$$

On the other hand we can improve (5.7). To do that, making covariant derivative $\stackrel{*}{\nabla}_{\alpha}$ on both sides of the first equation in (3.9) and combining last equation in (3.9), $\left(p_{0}, p_{1}, \mathbf{u}_{1}\right)$ can be found by

$$
-\frac{\delta^{2}}{2} \stackrel{*}{\Delta} p_{0}=-\mu \delta\left(\operatorname{div}\left(2 H \mathbf{u}_{0}\right)-2 K u_{0}^{3}-4 H \gamma_{0}\left(\mathbf{u}_{0}\right)\right)-a^{\alpha \beta} \stackrel{*}{\nabla}_{\alpha} F_{\beta}^{1}
$$

satisfies periodic boundary conditions on $\partial D$,

$$
\begin{aligned}
-\frac{\delta^{2}}{2} p_{1}= & \left(\delta-\frac{H \delta^{2}}{2}\right) p_{0}+\frac{\mu \delta^{2}}{2} \beta_{0}\left(\mathbf{u}_{0}\right)+\frac{\mu \delta^{2}}{2} \stackrel{*}{\Delta} u_{0}^{3}+\frac{\delta^{2}}{2}\left(u_{0}^{\lambda} \stackrel{*}{\nabla}_{\lambda} u_{0}^{3}+b_{\alpha \beta} u_{0}^{\alpha} u_{0}^{\beta}\right)+F_{3}^{1}, u_{1}^{3}=-\gamma_{0}\left(\mathbf{u}_{0}\right), \\
\mu \delta u_{1}^{\beta}= & \frac{\mu \delta^{2}}{2}\left[\left(\stackrel{*}{\Delta} u_{0}^{\alpha}+a^{\alpha \lambda} \stackrel{*}{\nabla}_{\lambda} \operatorname{div} \mathbf{u}_{0}+K u_{0}^{\alpha}\right)+4 a^{\alpha \lambda} \stackrel{*}{\nabla}_{\lambda} H u_{0}^{3}+2 b^{\alpha \beta} \stackrel{*}{\nabla}_{\beta} u_{0}^{3}\right]-\frac{\delta^{2}}{2} a^{\alpha \beta} \stackrel{\nabla}{\nabla}_{\beta} p_{0} \\
& +\frac{\delta^{2}}{2}\left(u_{0}^{\lambda} \stackrel{*}{\nabla}_{\lambda} u_{0}^{\alpha}-2 b_{\beta}^{\alpha} u_{0}^{\beta} u_{0}^{3}\right)+a^{\alpha \beta} F_{\beta}^{1},
\end{aligned}
$$


The variational formulations corresponding to (5.7) and (5.1) are given respectively by

$$
\left\{\begin{array}{l}
\text { Find }\left(\mathbf{u}_{0}, u_{0}^{3}\right) \in V(D) \times H_{p}^{1}(D) \text { such that } \\
\delta A_{0}\left(\left(\mathbf{u}_{0}, u_{0}^{3}\right),\left(\mathbf{v}_{0}, v_{0}^{3}\right)\right)+\delta b\left(\mathbf{u}_{0} ; \mathbf{u}_{0}, \mathbf{v}_{0}\right)+\delta \alpha_{0}\left(p_{0}, \operatorname{div}^{*} \mathbf{v}_{0}\right) \\
-2 \mu \delta \tau\left(\beta_{0}\left(\mathbf{u}_{0}\right), v_{0}^{3}\right)+\delta b\left(\mathbf{u}_{0} ; \mathbf{u}_{0}, v_{0}^{3}\right)-\tau \delta\left(2 H p_{0}, v_{0}^{3}\right) \\
=\alpha_{0}\left(\left(F_{\beta}, v_{0}^{\beta}\right)-\delta\left(u_{0}^{3} u_{1}^{\alpha}, a_{\alpha \beta} v_{0}^{\beta}\right)\right)+\tau\left(\delta \operatorname{div}_{1}^{*}+F_{3}^{0}, v_{0}^{3}\right), \quad \forall\left(\mathbf{v}_{0}, v_{0}^{3}\right) \in V(D) \times H_{p}^{1}(D),
\end{array}\right.
$$

and

$$
\left\{\begin{array}{l}
\text { Find } p_{0} \in H_{p}^{1}(D) \text { such that } \\
\delta \mu\left(\stackrel{*}{\nabla} p_{0}, \stackrel{*}{\nabla} q\right)=-\left(\mu \delta\left(\operatorname{div}^{*}\left(2 H \mathbf{u}_{0}\right)-2 K u_{0}^{3}-4 H \gamma_{0}\left(\mathbf{u}_{0}\right)\right)+a^{\alpha \beta} \stackrel{*}{\nabla}_{\alpha} F_{\beta}^{1}, q\right), \quad \forall q \in H_{p}^{1}(D),
\end{array}\right.
$$

where the bilinear forms and linear form are defined by

$$
\left\{\begin{array}{l}
A_{0}\left(\mathbf{u}_{0}, \mathbf{v}_{0}\right):=\alpha_{0} \delta\left(a_{0}^{\alpha \beta \lambda \sigma} \gamma_{\lambda \sigma}\left(\mathbf{u}_{0}\right), \gamma_{\alpha \beta}\left(\mathbf{v}_{0}\right)\right)+2 \mu \tau \delta\left(\stackrel{*}{\nabla} u_{0}^{3}, \stackrel{*}{\nabla} v_{0}^{3}\right), \\
b\left(\mathbf{u}_{0} ; \mathbf{u}_{0}, \mathbf{v}_{0}\right):=\alpha_{0}\left(a_{\alpha \beta} u_{0}^{\lambda} \stackrel{*}{\nabla}_{\lambda} u_{0}^{\alpha}-2 b_{\alpha \beta} u_{0}^{\alpha} u_{0}^{3}, v_{0}^{\beta}\right), \\
b_{0}\left(\mathbf{u}_{0} ; \mathbf{u}_{0}, v_{0}^{3}\right)=\tau\left(u_{0}^{\lambda} \stackrel{*}{\nabla}_{\lambda} u_{0}^{3}+b_{\lambda \sigma} u_{0}^{\lambda} u_{0}^{\sigma}, v_{0}^{3}\right),
\end{array}\right.
$$

(III) For $i=N$ i.e. a last artificial interface Surface $\mathfrak{I}_{N}$. There are two choices to do that (1) assume that $\mathbf{u}=\mathbf{u}_{\infty}$ on $\mathfrak{I}_{N}$ where $\mathbf{u}_{\infty}$ is known infinity up stream flow velocity. (2) we assume that the flow outside $\mathfrak{I}_{N}$ is governed by Oseen equation and give a boundary integrating equation on $\mathfrak{I}_{N}$ via fundamental solution of Oseen equations.

(1) Let $(x, y, z)$ is Cartesian coordinate and $\mathbf{u}_{\infty}=u_{\infty} \mathbf{k}$ where $(\mathbf{i}, \mathbf{j}, \mathbf{k})$ are base vectors. The surface $\mathfrak{I}_{N}$ can be parametrization by $\mathbf{r}=x\left(x^{1}, x^{2}\right) \mathbf{i}+y\left(x^{1}, x^{2}\right) \mathbf{j}+z\left(x^{1}, x^{2}\right) \mathbf{k}$ where $x^{\alpha}$ are parameters, i.e. are Gaussian coordinate on $\mathfrak{I}_{N}$. Then base vectors $\boldsymbol{e}_{\alpha}$ and unit normal vector $n$ in semi-coordinate on $\mathfrak{I}_{N}$ are given by

$$
\left\{\begin{array}{l}
\mathbf{e}_{\alpha}=x_{\alpha} \mathbf{i}+y_{\alpha} \mathbf{j}+z_{\alpha} \mathbf{k} \\
\mathbf{n}=\frac{1}{\sqrt{a}}\left(\mathbf{e}_{1} \times \mathbf{e}_{2}\right)=\frac{1}{\sqrt{a}}\left[\left(y_{1} z_{2}-z_{1} y_{2}\right) \mathbf{i}+\left(z_{1} x_{2}-z_{2} x_{1}\right) \mathbf{j}+\left(x_{1} y_{2}-y_{1} x_{2}\right) \mathbf{k}\right]
\end{array}\right.
$$

while the metric tensor $a_{\alpha \beta}$ and curvature tensor $b_{\alpha \beta}$ are given

$$
\left\{\begin{array}{l}
a_{\alpha \beta}=x_{\alpha} x_{\beta}+y_{\alpha} y_{\beta}+z_{\alpha} z_{\beta}, \quad a=\operatorname{det}\left(a_{\alpha \beta}\right), \\
b_{\alpha \beta}=\frac{1}{\sqrt{a}}\left[\begin{array}{ccc}
x_{\alpha \beta} & y_{\alpha \beta} & z_{\alpha \beta} \\
x_{\alpha} & y_{\alpha} & z_{\alpha} \\
x_{\beta} & y_{\beta} & z_{\beta}
\end{array}\right], \quad c_{\alpha \beta}=a^{\lambda \sigma} b_{\alpha \lambda} b_{\beta \sigma},
\end{array}\right.
$$

where $x_{\alpha}=\frac{\partial x}{\partial x^{\alpha}}, x_{\alpha \beta}=\frac{\partial^{2} x}{\partial x^{\alpha} \partial x^{\beta}}$. Our aim is to give boundary conditions on $\mathfrak{J}_{N}$. Owing to (4.12) we claim

$$
\begin{aligned}
& h_{t}^{\alpha}=\mu\left(\left.\frac{\partial u_{\infty}^{\alpha}}{\partial \xi}\right|_{\xi=0}+a^{\alpha \beta} \nabla_{\beta}^{*} u_{\infty}^{3}-b_{\beta}^{\alpha} u_{\infty}^{\beta}\right), \\
& h_{t}^{3}=-\left.p\right|_{\mathfrak{I}_{N}}+\left.2 \mu \frac{\partial u_{\infty}^{3}}{\partial \xi}\right|_{\xi=0}=-\frac{3}{2} \mathbf{u}_{\infty} \operatorname{grad} \frac{1}{r}+\left.2 \mu \frac{\partial u_{\infty}^{3}}{\partial \xi}\right|_{\xi=0},
\end{aligned}
$$


On other hand, we show

$$
\left\{\begin{array}{l}
\left.\frac{\partial u_{\infty}^{\alpha}}{\partial \xi}\right|_{\xi=0}=2 b^{\alpha \beta} z_{\beta} u_{\infty},\left.\quad \frac{\partial u_{\infty}^{3}}{\partial \xi}\right|_{\xi=0}=0, \\
\left.u_{\infty}^{\alpha}\right|_{\xi=0}=a^{\alpha \beta} u_{\infty \beta}=a^{\alpha \beta} u_{\infty} \mathbf{k} \mathbf{e}_{\beta}=a^{\alpha \beta} z_{\beta} u_{\infty}, \\
\left.u_{\infty}^{3}\right|_{\xi=0}=u_{\infty} \mathbf{k n}=\frac{u_{\infty}}{\sqrt{a}}\left(x_{1} y_{2}-y_{1} x_{2}\right) .
\end{array}\right.
$$

Indeed,

$$
\begin{aligned}
& u_{\infty}^{\alpha}=g^{\alpha \beta} u_{\infty \beta}=g^{\alpha \beta} \mathbf{k} \cdot \mathbf{e}_{\beta} u_{\infty}=g^{\alpha \beta} z_{\beta} u_{\infty}, \\
& \left.\frac{\partial g^{\alpha \beta}}{\partial \xi}\right|_{\xi=0}=2 b_{\alpha \beta}(\operatorname{see}(1.8 .23) \operatorname{in}[1]),\left.\quad \frac{\partial u_{\infty}^{\alpha}}{\partial \xi}\right|_{\xi=0}=\frac{\partial g^{\alpha \beta}}{\partial \xi} z_{\beta} u_{\infty}=2 b^{\alpha \beta} x_{\beta} u_{\infty}, \\
& u_{\infty}^{3}=u_{\infty} \mathbf{k n}=\frac{u_{\infty}}{\sqrt{a}}\left(x_{1} y_{2}-y_{1} x_{2}\right),\left.\quad \frac{\partial u_{\infty}^{3}}{\partial \xi}\right|_{\xi=0}=\frac{\partial u_{\infty}}{\partial \xi}=0 .
\end{aligned}
$$

Finally we imply

$$
h_{t}^{\alpha}(N)=\mu u_{\infty}\left(a^{\alpha \beta} \partial_{\beta}\left(\frac{x_{1} y_{2}-y_{1} x_{2}}{\sqrt{a}}\right)+b^{\alpha \beta} z_{\beta}\right), \quad h_{t}^{3}(N)=\frac{3}{2} u_{\infty} \operatorname{kgrad} \frac{1}{r}=-\frac{3}{2} u_{\infty} \frac{z\left(x^{\alpha}\right)}{r}
$$

where $\mathbf{r}=\mathbf{r}\left(x^{\alpha}\right)$ is describing $\mathfrak{I}_{N}$. (5.16) will be used for solving BLE I on $\mathfrak{I}_{N-1}$ with $\mathbf{h}_{t}$.

(2). Let assume that the flow outside of $\mathfrak{I}_{N}$ is governed by Oseen equation

$$
\left\{\begin{array}{l}
-\Delta \mathbf{u}+\mathbf{b} \cdot \nabla \mathbf{u}+\nabla p=f, \quad \text { in } \tilde{\Omega}, \\
\operatorname{divu}=0, \quad \text { in } \widetilde{\Omega}, \\
\left.\mathbf{u}\right|_{\mathfrak{I}_{m}} ^{+}=\left.\mathbf{u}\right|_{\mathfrak{I}_{m}} ^{-}=\mathbf{u}_{0}+\delta \mathbf{u}_{1},\left.\quad \sigma(\mathbf{u}, \mathbf{p})\right|_{\mathfrak{I}} ^{+}=\left.\sigma(\mathbf{u}, \mathbf{p})\right|_{\mathfrak{I}} ^{-} \text {on } \mathfrak{I}_{m}, \\
\mathbf{u} \rightarrow \mathbf{u}_{\infty}, \text { at } \quad \mathbf{x} \rightarrow \infty,
\end{array}\right.
$$

$\mathbf{u}_{\infty}$ is known and $\mathbf{b}$ is a well known vector, for example $\mathbf{b}=\mathbf{u}_{\infty}$, and $\hat{\Omega}=\tilde{\Omega} \cup \sum_{i=0}^{m} \Omega_{i, i+1}$, and $\mathbf{u}_{0}$, $\mathbf{u}_{1}$ are solutions of 2D-3C Navier-Stokes equations on the $\mathfrak{I}_{m-1}$. Furthermore, $\left.\sigma(\mathbf{u}, \mathbf{p})\right|_{\mathfrak{I}} ^{+}=\mathbf{h}$ is normal stress tensor to be found in the section. Let $\mathbf{x}$ be a Cartesian coordinate. $\mathcal{U}_{k i}, \mathcal{P}_{k}$ are fundamental solutions of the following equations

$$
\left\{\begin{array}{l}
-v \Delta \mathcal{U}_{k i}(\mathbf{x}-\mathbf{y})+\frac{\partial \mathcal{P}_{k}}{\partial y_{i}}(\mathbf{x}-\mathbf{y})+b \cdot \nabla \mathcal{U}_{k i}(\mathbf{x}-\mathbf{y})=-\delta_{k i} \delta(\mathbf{x}-\mathbf{y}) \\
\frac{\partial \mathcal{U}_{k i}}{\partial y_{i}}(\mathbf{x}-\mathbf{y})=0 .
\end{array}\right.
$$

$\left(\mathcal{U}_{k i}, \mathcal{P}_{k}\right)$ can be expressed as

$$
v \mathcal{U}_{k i}(x-y)=\delta_{k i} \Delta \Phi(x-y)-\frac{\partial^{2} \Phi(x-y)}{\partial x_{k} \partial x_{i}}, \quad \mathcal{P}_{k}(x-y)=-\frac{\partial}{\partial x_{k}}(\Delta+\mathbf{b} \cdot \nabla) \Phi(x-y)
$$

where $\Phi$ is a fundamental solution of following equation

$$
\left(\Delta+\mathbf{u}_{\infty} \cdot \nabla\right) \Delta \Phi=\delta(\mathbf{x}-\mathbf{y}), \quad \Phi(x-y)=-\frac{1}{|\mathbf{b}|} \int_{0}^{\frac{\mathbf{b} \cdot(x-y)}{|\mathbf{b}|}}\left[\Phi_{2}-\Phi_{1}\right] \mathrm{d} t
$$

where for $\mathbf{b} \neq 0$ 


$$
\begin{aligned}
& \Phi_{1}(x-y)=-\frac{1}{2 \pi}\left(\frac{|\mathbf{b}|}{4 \pi|x-y|}\right)^{\frac{n-2}{2}} K_{\frac{n-2}{2}}\left(\frac{|\mathbf{b}||x-y|}{2}\right) \exp \left(-\frac{1}{2} \mathbf{b} \cdot(\mathbf{x}-\mathbf{y})\right), \quad n \geq 3 \\
& \Phi_{2}(x-y)= \begin{cases}\frac{\Gamma\left(\frac{n}{2}\right)}{2 \pi^{\frac{n}{2}}}(n-2)|x-y|^{n-2}, & n \geq 3, \\
\frac{1}{2 \pi} \ln |x-y|, & n=2,\end{cases}
\end{aligned}
$$

where $K_{\alpha}$ is a Bessel function of second kind.

Then integral expressions of solutions of Oseen problem (5.9) are given by

$$
\left\{\begin{array}{l}
\eta(y) u(\mathbf{y})=\mathbf{u}_{\infty}-\int_{\Gamma_{\mathrm{Art}}}[\mathcal{U} \cdot \sigma(\mathbf{u}, p)-\mathbf{u} \cdot \sigma(\mathcal{U}, \mathcal{P})] \mathrm{d} s, \\
\eta(y) p(\mathbf{y})=p_{\infty}-\int_{\Gamma_{\mathrm{Art}}}[\mathcal{P} \cdot \sigma(\mathbf{u}, p)-\mathbf{u} \cdot \sigma(\mathcal{U}, \mathcal{P})] \mathrm{d} s
\end{array}\right.
$$

where $\sigma$ is stress tensor

$$
\begin{gathered}
\sigma_{i j}(\mathbf{u}, p)=\left\{-\delta_{i j} p+\mu\left(\partial_{i} u^{j}+\partial_{j} u^{i}\right)\right\} \\
\eta(x)= \begin{cases}1, & x \in \text { Oseen Domain } \\
\frac{1}{2}, & x \in \text { Artificial Surface. }\end{cases}
\end{gathered}
$$

Here we employ Cartesian coordinate system $\left(\mathbf{x}=\left(x^{1}, x^{2}, x^{3}\right)\right)$ and artificial surface $\Gamma_{A r t}=\mathfrak{I}_{m}$ is a two dimensional manifold. The integrate representation (5.17) of the solution of Oseen problem is invariant, it is valid for any curvature coordinate. Since formula for fundamental solution cal $\mathcal{U}, \mathcal{P}$ is represent at Cartesian coordinate. It also can be compute at any curvature coordinate according transformation rule of tensor of one order.

Vector $\lambda$ in (5.17) is normal stress tensor at $\mathfrak{I} \lambda(\mathbf{u}, p)=\left\{\sigma_{i j}(\mathbf{u}, p) n^{j}, i=1,2,3\right\}$ The normal stress tensor $\lambda$ at $\mathfrak{I}_{m}$ is continuous $\left.\lambda\right|_{\mathfrak{I}_{m}} ^{+}=\left.\lambda\right|_{\mathfrak{I}_{m}} ^{-}=\mathbf{h}$. This means that $\lambda$ on both sides of $\mathfrak{I}_{m}$ are coincidental.

Normal stress tensor $\mathbf{h}$ on the artificial boundary $\mathfrak{I}_{m}$ satisfies following equation

$$
\begin{aligned}
& 2 c(\mathbf{h}, \chi)-\langle\mathbf{u}, \chi\rangle+2\langle K \mathbf{u}, \chi\rangle=0, \quad \forall \chi \in H^{-1 / 2}\left(\mathfrak{I}_{m}\right), \\
& c(\mathbf{h}, \chi)=\int_{\mathfrak{\Im}_{m}} \int_{\mathfrak{\Im}_{m}} h_{i} \mathcal{U}_{i j} \chi_{j} \mathrm{~d} s_{\chi} \mathrm{d}_{y} s, \quad\langle\mathbf{u}, \chi\rangle=\int_{\mathfrak{\Im}_{m}} u_{i} \chi_{i} \mathrm{~d} s, \\
& \langle K \mathbf{u}, \chi\rangle \int_{\mathfrak{\Im}_{m}} \int_{\Im_{m}}(\mathbf{u} \cdot \sigma(\mathcal{U}, \mathcal{P}) \cdot \chi) \mathrm{d} s,
\end{aligned}
$$

(5.23) can be rewrite in semi-geodesic coordinate based on $\mathfrak{I}_{m-1}$ :

$$
\begin{aligned}
& 2 c(\mathbf{h}, \chi)-\langle\mathbf{u}, \chi\rangle+2\langle K \mathbf{u}, \chi\rangle=0, \quad \forall \chi \in\left(H^{-1 / 2}\left(\mathfrak{I}_{m}\right)\right)^{3}, \\
& c(\mathbf{h}, \chi)=\iint_{D D} \tilde{\mathcal{U}}_{i j} u_{0}^{i} \chi^{j} \sqrt{a} \mathrm{~d} x, \quad\langle\mathbf{u}, \chi\rangle=\iint_{D}\left[a_{\alpha \beta} u_{0}^{\alpha} \chi^{\gamma}+u^{3} \chi^{3}\right] \sqrt{a} \mathrm{~d} x, \\
& \langle K \mathbf{u}, \chi\rangle=\iint_{D D} \sigma_{i j}(\tilde{\mathcal{U}}, \tilde{\mathcal{P}}) u_{0}^{i} \chi^{j} \sqrt{a} \mathrm{~d} x,
\end{aligned}
$$

where, $\left(x^{\alpha}, x^{3}=\xi\right)$ is semi-geodesic coordinate. By the transformation of coordinate, $\tilde{\mathcal{U}}_{i j^{\prime}}=\mathcal{U}_{i j} \frac{\partial X^{i}}{\partial x^{i^{\prime}}} \frac{\partial X^{j}}{\partial x^{j^{\prime}}}$ where $X^{i}$ are Cartesian coordinate and $X^{i}=X^{i}(x)$ is the parametrization representation of the surface $\mathfrak{I}_{m-1}$. 
Lemma 5 The bilinear form $c(\cdot, \cdot)$ defined by (5.23) is symmetric, continuous and coercive from $\mathbf{H}^{-1 / 2}\left(\mathfrak{I}_{m}\right) \times \mathbf{H}^{-1 / 2}\left(\mathfrak{I}_{m}\right)$ into $\mathfrak{R}^{3}$

$$
\begin{aligned}
& c\left(\chi_{1}, \chi_{2}\right)=c\left(\chi_{2}, \chi_{1}\right), \quad \forall \chi_{1}, \chi_{2} \in \mathbf{H}^{-1 / 2}\left(\mathfrak{I}_{m}\right), \\
& \left|c\left(\chi_{1}, \chi_{2}\right)\right| \leq C\left\|\chi_{1}\right\|_{-1 / 2, \mathfrak{I}_{m}}\left\|\chi_{2}\right\|_{-1 / 2, \mathfrak{I}_{m}}, \quad \forall \chi_{1}, \chi_{2} \in \mathbf{H}^{-1 / 2}\left(\mathfrak{I}_{m}\right), \\
& \left|c\left(\chi_{1}, \chi_{1}\right)\right| \geq C\left\|\chi_{1}\right\|_{-1 / 2, \mathfrak{I}_{m}}^{2}, \quad \forall \chi_{1} \in \mathbf{H}^{-1 / 2}\left(\mathfrak{I}_{m}\right),
\end{aligned}
$$

Theorem 4 Assume that $\mathbf{u}_{0}, p_{0}$ are smooth and bounded in $\mathbf{H}_{p}^{1}(D)$ Then there exists a unique solution of following variational problem

$$
\left\{\begin{array}{l}
\text { Find } \mathbf{h} \in \mathbf{H}_{p}^{-1 / 2}(D) \text { such that } \\
c(\mathbf{h}, \chi)=\frac{1}{2}\left\langle\mathbf{u}_{0}, \chi\right\rangle-\left\langle K \mathbf{u}_{0}, \chi\right\rangle, \quad \forall \chi \in \mathbf{H}^{-1 / 2}\left(\mathfrak{I}_{m}\right),
\end{array}\right.
$$

Parallel algorithms. The Domain ia made partition by m interfaces surfaces and we obtain $m+1$ the systems of BLE I and SLE II. Solving each BLE I and SLE II independently, then applying alternatively iterative algorithm are performance at the same time. On the other hand, the parallel algorithms for BLE I and SLE II can be used. Therefore, parallel algorithms are applied in two direction at the same time.

\section{Computation of the Drag}

The drag is a force exerted on a solid boundary surface, for example, $\mathfrak{I}_{0}$. There is normal stress on $\mathfrak{I}_{0}$ which can expressed under semi-geodesic coordinate based on $\mathfrak{I}$ by

$$
\int_{\Im_{0}} \sigma^{i j}(\mathbf{u}, p) n_{i} \sqrt{a} \mathrm{~d} x
$$

The drag is a projection of normal stress on the direction of infinite stream flow $\mathbf{u}_{\infty}=u_{\infty} \mathbf{k}$. Hence

$$
F_{d}=\int_{\Im_{0}} \sigma^{i j}(\mathbf{u}, p) n_{i} \mathbf{k}_{j} \sqrt{a} \mathrm{~d} x
$$

Since unit normal vector at $\mathfrak{I}_{0}$ is $\mathbf{n}=(0,0,1)$ and by $(5.1)$

$$
k_{\alpha}=\mathbf{k e}_{\alpha}=z_{\alpha}, \quad k_{3}=\mathbf{k} n=\frac{x_{1} y_{2}-x_{2} y_{1}}{\sqrt{a}}
$$

Therefore

$$
\sigma^{i j}(\mathbf{u}, p) n_{i} k_{j}=\sigma^{3 \alpha}(\mathbf{u}, p) z_{\alpha}+\sigma^{33}(\mathbf{u}, p) \frac{x_{1} y_{2}-x_{2} y_{1}}{\sqrt{a}}
$$

As well known that the stress tensor is given by

$$
\sigma^{i j}(\mathbf{u}, p)=-p g^{i j}+2 \mu g^{i l} g^{j m} e_{l m}(\mathbf{u})
$$

At surface $\mathfrak{I}_{0}$

$$
\begin{gathered}
\left.\sigma^{33}(\mathbf{u}, p)\right|_{\mathfrak{I}_{0}}=-p_{0}+\left.2 \mu e_{33}(\mathbf{u})\right|_{\mathfrak{I}_{0}}=-p_{0}+\left.2 \mu \frac{\partial u^{3}}{\partial \xi}\right|_{\xi=0}=-p_{0}+2 \mu u_{1}^{3}=-p_{0} \\
\left.\sigma^{3 \alpha}(\mathbf{u}, p)\right|_{\mathfrak{I}_{0}}=2 \mu a^{\alpha \beta} e_{3 \beta}\left(u_{0}\right)
\end{gathered}
$$

Since

because of

$$
\begin{aligned}
e_{3 \beta}(\mathbf{u}) & =\frac{1}{2}\left(g_{\beta m} \nabla_{3} u^{m}+g_{33} \nabla_{\beta} u^{3}\right)=\frac{1}{2}\left(g_{\beta \sigma}\left(\frac{\partial u^{\sigma}}{\partial \xi}+\theta^{-1} I_{\lambda}^{\sigma} u^{\lambda}\right)+\stackrel{\nabla}{\nabla}_{\beta} u^{3}+J_{\beta \lambda} u^{\lambda}\right) \\
& =\frac{1}{2}\left(g_{\beta \sigma} \frac{\partial u^{\sigma}}{\partial \xi}+\stackrel{*}{\nabla}_{\beta} u^{3}\right)+\frac{1}{2}\left(\theta^{-1} g_{\beta \lambda} I_{\lambda}^{\sigma}+J_{\beta \lambda}\right) u^{\lambda}=\frac{1}{2}\left(g_{\beta \sigma} \frac{\partial u^{\sigma}}{\partial \xi}+\nabla_{\beta}^{*} u^{3}\right),
\end{aligned}
$$




$$
\begin{aligned}
& \theta^{-1} g_{\beta \lambda} I_{\lambda}^{\sigma}+J_{\beta \lambda}=0, \quad \text { see Lemma 1.8.1(Li[1]), } \\
& \left.\mathbf{u}\right|_{\mathfrak{I}_{0}}=0,\left.\quad u_{1}^{3}\right|_{\mathfrak{I}_{0}}=0,\left.\quad e_{3 \beta}(\mathbf{u})\right|_{\mathfrak{I}_{0}}=\frac{1}{2} a_{\beta \lambda} u_{1}^{\lambda}, \\
& \left.\sigma^{3 \alpha}(\mathbf{u}, p)\right|_{\mathfrak{I}_{0}}=\mu u_{1}^{\alpha} .
\end{aligned}
$$

Hence

$$
F_{d}=\int_{\mathfrak{\Im}_{0}}\left(-p_{0} \frac{x_{1} y_{2}-x_{2} y_{1}}{\sqrt{a}}+\mu u_{1}^{\alpha} z_{\alpha}\right) \sqrt{a} \mathrm{~d} x
$$

The drag is a force exerted on a solid boundary surface, for example, $\mathfrak{I}_{0}$. There is normal stress on $\mathfrak{I}_{0}$ which can be expressed under semi-geodesic coordinate based on $\mathfrak{I}$ by $\int_{\mathfrak{I}_{0}} \sigma^{i j}(\mathbf{u}, p) n_{i} \sqrt{a} \mathrm{~d} x$. The drag is a projection of normal stress on the direction of infinite stream flow $\mathbf{u}_{\infty}=u_{\infty} \mathbf{k}$. Hence

$$
F_{d}=\int_{\Im_{0}} \sigma^{i j}(\mathbf{u}, p) n_{i} \mathbf{k}_{j} \sqrt{a} \mathrm{~d} x=\int_{\Im_{0}}\left(-p_{0} \frac{X_{1} Y_{2}-X_{2} Y_{1}}{\sqrt{a}}+\mu u_{1}^{\alpha} Z_{\alpha}\right) \sqrt{a} \mathrm{~d} x
$$

where $X(x), Y(x), Z(x)$ are parameter representation of $\mathfrak{J}_{0}$.

\section{Examples}

\subsection{The Flow around a Sphere}

Assume that $(x, y, z)$ and $\left(x^{3}=r, x^{2}=\varphi, x^{1}=\theta\right)$ are Cartesian and spherical coordinates respectively

$$
x=r \sin \theta \cos \varphi, \quad y=r \sin \theta \sin \varphi, \quad z=r \cos \theta, \quad\left(g_{i j}\right)=\left(\begin{array}{ccc}
1 & 0 & 0 \\
0 & r^{2} \sin ^{2} \theta & 0 \\
0 & 0 & r^{2}
\end{array}\right)
$$

Simple calculations show that the metric tensor of spherical surface $r=$ const . is given

$$
\left\{\begin{array}{l}
a_{11}=r^{2}, \quad a_{12}=a_{21}=0, \quad a_{22}=r^{2} \sin ^{2} \theta, \quad a=\operatorname{det} a_{\alpha \beta}=r^{4} \sin ^{2} \theta, \\
a^{11}=r^{-2}, \quad a^{12}=a^{21}=0, \quad a^{22}=\frac{1}{r^{2} \sin ^{2} \theta},
\end{array}\right.
$$

The tensor of second fundamental form, i.e. curvature tensor of spherical surface is given by

$$
\left\{\begin{array}{l}
b_{11}=r, \quad b_{22}=r \sin ^{2} \theta, \quad b_{12}=0, \quad b^{22}=\frac{1}{r^{3} \sin ^{3} \theta}, \quad b^{11}=\frac{1}{r^{3}}, \quad b^{12}=0, \\
b_{1}^{1}=b_{2}^{2}=\frac{1}{r}, \quad b_{2}^{1}=b_{1}^{2}=0, \quad b=\operatorname{det}\left(b_{\alpha \beta}\right)=r^{2} \sin ^{2} \theta, \\
K=\frac{b}{a}=\frac{1}{r^{2}}, \quad H=a^{\alpha \beta} b_{\alpha \beta}=\frac{1}{r},
\end{array}\right.
$$

the base vectors of semi-geophysical coordinate system are given

$$
\left\{\begin{array}{l}
\mathbf{e}_{1}=-r \cos \varphi \cos \theta \mathbf{i}+r \cos \theta \sin \varphi \mathbf{j}-r \sin \theta \mathbf{k}, \\
\mathbf{e}_{2}=-r \sin \varphi \sin \theta \mathbf{i}+r \sin \theta \cos \varphi \mathbf{j}, \\
\mathbf{n}=\frac{\mathbf{r}}{r},
\end{array}\right.
$$

We remainder have to give the covariant derivatives of the velocity field, Laplace-Betrami operator and trace-Laplace operator. To do this we have to give the first and second kind of Christoffel symbols on the spherical surface $\mathfrak{I}$ as a two dimensional manifolds 


$$
\begin{aligned}
& \stackrel{*}{\Gamma}_{12,2}=\stackrel{*}{\Gamma}_{21,2}=r^{2} \sin \theta \cos \theta, \quad \stackrel{*}{\Gamma}_{22,1}=-r^{2} \sin \theta \cos \theta, \quad \stackrel{*}{\Gamma}_{\alpha \beta, \gamma}=0, \quad \text { other way, } \\
& \stackrel{*}{\Gamma}^{2}=\stackrel{*}{\Gamma}^{2}{ }_{21}=\cot \theta, \quad \stackrel{*}{\Gamma}^{1}{ }_{22}=-\sin \theta \cos \theta, \quad \stackrel{*}{\Gamma}_{\alpha \beta}^{\gamma}=0, \quad \text { other way, }
\end{aligned}
$$

Then covariant derivatives of vector $\mathbf{u}=u^{\alpha} \mathbf{e}_{\alpha}+u^{3} \mathbf{n}$ on the two dimensional manifold $\mathfrak{I}$ is given by

$$
\left\{\begin{array}{l}
\stackrel{*}{\nabla}_{1} u^{1}=\partial_{\theta} u^{1}, \quad \stackrel{*}{\nabla}_{2} u^{1}=\partial_{\varphi} u^{1}+\Gamma^{1}{ }_{2 \lambda} u^{\lambda}=\partial_{\varphi} u^{1}-\sin \theta \cos \theta u^{2}, \\
\stackrel{*}{\nabla}_{1} u^{2}=\partial_{\theta} u^{2}+\cot \theta u^{2}, \quad \stackrel{*}{\nabla}_{2} u^{2}=\partial_{\varphi} u^{2}+\cot \theta u^{1}, \\
\operatorname{*iv}^{\operatorname{div}}=\stackrel{*}{\nabla}_{\lambda} u^{\lambda}=\partial_{\theta} u^{1}+\partial_{\varphi} u^{2}+\cot \theta u^{1}, \\
a^{1 \beta} \stackrel{*}{\nabla}_{\beta} \operatorname{div} \mathbf{u}=\frac{1}{r^{2}}\left(\frac{\partial^{2} u^{1}}{\partial \theta^{2}}+\frac{\partial^{2} u^{2}}{\partial \theta \partial \varphi}+\cot \theta \frac{\partial u^{1}}{\partial \theta}-\frac{1}{\sin ^{2} \theta} u^{1}\right), \\
a^{2 \beta} \stackrel{*}{\nabla}_{\beta} \operatorname{div} \mathbf{u}=\frac{1}{r^{2} \sin ^{2} \theta}\left(\frac{\partial^{2} u^{1}}{\partial \theta \partial \varphi}+\frac{\partial^{2} u^{2}}{\partial \varphi^{2}}+\cot \theta \frac{\partial u^{1}}{\partial \varphi}\right),
\end{array}\right.
$$

Nonlinear terms

$u^{\lambda} \nabla_{\lambda}^{*} u^{1}-u^{1} \operatorname{div}_{\mathbf{u}}^{*}=u^{2} \frac{\partial u^{1}}{\partial \varphi}-u^{1} \frac{\partial u^{2}}{\partial \varphi}-\frac{1}{2} \sin 2 \theta u^{2} u^{2}-\cot \theta u^{1} u^{1}, u^{\lambda} \nabla_{\lambda}^{*} u^{2}-u^{2} \operatorname{div} \mathbf{u}=u^{1} \frac{\partial u^{2}}{\partial \theta}-u^{2} \frac{\partial u^{1}}{\partial \theta}+\cot \theta u^{1} u^{2}$, and

$$
Q_{11}=2 \mu\left(\frac{1}{8} \delta r^{2}+\frac{2 r \delta^{2}}{2}+\frac{5}{8} \frac{\delta^{3}}{3}\right), \quad Q_{22}=Q_{11} \sin ^{2} \theta, \quad Q_{12}=0, M_{0}\left(\mathbf{u}_{1}\right)=\frac{\mu \delta^{2}}{2}\left(\partial_{\theta} u_{1}^{1}+\partial_{\varphi} u_{1}^{2}+\cot \theta u_{1}^{1}\right)
$$

The associated Laplace-Betrami operator and divergence operator on $\mathfrak{I}$ are given by

$$
\stackrel{*}{\Delta} p_{0}=\frac{1}{r^{2} \sin ^{2} \theta}\left[\sin ^{2} \theta \frac{\partial^{2} p_{0}}{\partial \theta^{2}}+\frac{\partial^{2} p_{0}}{\partial \varphi^{2}}+\cot \theta \frac{\partial p_{0}}{\partial \theta}\right]
$$

while trace-Laplace operator on $\mathfrak{I}$

$$
\left\{\begin{array}{l}
\Delta^{1} u^{1}=\frac{1}{r^{2}} \frac{\partial^{2} u^{1}}{\partial \theta^{2}}+\frac{1}{r^{2}} \sin ^{2} \theta \frac{\partial^{2} u^{1}}{\partial \theta \partial \varphi}+\frac{\cot \theta}{r^{2}} \frac{\partial u^{1}}{\partial \theta}-\frac{2 \cot \theta}{r^{2}} \frac{\partial u^{2}}{\partial \varphi}-\frac{\cot ^{2} \theta}{r^{2}} u^{1}, \\
\Delta^{2} u^{2}=\frac{1}{r^{2}} \frac{\partial^{2} u^{2}}{\partial \theta^{2}}+\frac{1}{r^{2}} \sin ^{2} \theta \frac{\partial^{2} u^{2}}{\partial \varphi^{2}}+\frac{\cot \theta}{r^{2}} \sin ^{2} \theta \frac{\partial u^{1}}{\partial \theta}+\frac{3 \cot \theta}{r^{2}} \frac{\partial u^{2}}{\partial \theta}+\frac{\cot \theta}{r^{2}} \sin ^{2} \theta \frac{\partial u^{2}}{\partial \varphi}-\frac{u^{2}}{r^{2}}
\end{array}\right.
$$

(A) BLE I

Substituting previous formula into Theorem 1 we assert that

$$
\left\{\begin{array}{l}
-\frac{\mu \delta^{3}}{3}\left[\frac{2}{r^{2}}\left(\frac{\partial^{2} u_{1}^{1}}{\partial \theta^{2}}+\frac{\partial^{2} u_{1}^{2}}{\partial \theta \partial \varphi}\right)+\frac{1}{r^{2}} \sin ^{2} \theta \frac{\partial^{2} u_{1}^{1}}{\partial \theta \partial \varphi}+\frac{2 \cot \theta}{r^{2}}\left(\frac{\partial u_{1}^{1}}{\partial \theta}-\frac{\partial u_{1}^{2}}{\partial \varphi}\right)-2 \cot ^{2} \theta u_{1}^{1}\right]+\frac{\mu}{2}\left(\delta-\frac{4}{r} \frac{\delta^{2}}{2}+\frac{6}{r^{2}} \frac{\delta^{3}}{3}\right) u_{1}^{1} \\
+\left(\frac{1}{r^{2}} \frac{3}{2} \frac{\delta^{2}}{2}-\frac{4 \delta^{3}}{3} \frac{1}{r^{3}}\right) \frac{\partial p_{0}}{\partial \theta}+\frac{\delta^{4}}{4}\left[u_{1}^{2} \frac{\partial u_{1}^{1}}{\partial \varphi}-u_{1}^{1} \frac{\partial u_{1}^{2}}{\partial \varphi}-\frac{1}{2} \sin 2 \theta u_{1}^{2} u_{1}^{2}-\cot \theta u_{1}^{1} u_{1}^{1}\right]=\frac{1}{r^{2}} F_{1}^{1}-\frac{\delta^{3}}{3} \frac{1}{r^{2}} \frac{\partial p_{1}}{\partial \theta}+\mathscr{F}^{1}\left(\mathbf{u}_{2}\right) \\
-\frac{\mu \delta^{3}}{3}\left[\frac{1}{r^{2}} \frac{\partial^{2} u_{1}^{2}}{\partial \theta^{2}}+\frac{1}{r^{2}} \sin ^{2} \theta \frac{\partial^{2} u_{1}^{2}}{\partial \varphi^{2}}+\frac{\cot \theta}{r^{2}} \sin ^{2} \theta\left(\frac{\partial u_{1}^{1}}{\partial \theta}+\frac{\partial u_{1}^{1}}{\partial \varphi}\right)+\frac{3 \cot \theta}{r^{2}} \frac{\partial u_{1}^{2}}{\partial \theta}+\frac{\cot \theta}{r^{2}} \sin ^{2} \theta \frac{\partial u_{1}^{2}}{\partial \varphi}\right] \\
+\frac{\mu}{2} \sin ^{2} \theta\left(\delta-\frac{4}{r} \frac{\delta^{2}}{2}+\frac{6}{r^{2}} \frac{\delta^{3}}{3}\right) u_{1}^{2}+\left(\frac{1}{r^{2}} \sin ^{2} \theta \frac{3}{2} \frac{\delta^{2}}{2}-\frac{4 \delta^{3}}{3} \frac{1}{r^{3}} \sin ^{3} \theta\right) \frac{\partial p_{0}}{\partial \varphi} \\
+\frac{\delta^{4}}{4}\left(u_{1}^{1} \frac{\partial u_{1}^{2}}{\partial \theta}-u_{1}^{2} \frac{\partial u_{1}^{1}}{\partial \theta}+\cot \theta u_{1}^{1} u_{1}^{2}\right)=\frac{1}{r^{2}} \sin ^{2} \theta F_{2}^{1}-\frac{\delta^{3}}{3} \frac{1}{r^{2}} \sin ^{2} \theta \frac{\partial p_{1}}{\partial \varphi}+\mathscr{T}^{2}\left(\mathbf{u}_{2}\right) \\
-\frac{\delta^{3}}{3}\left(\frac{1}{r^{2}} \frac{\partial^{2} p_{0}}{\partial \theta^{2}}+\frac{1}{r^{2}} \sin ^{2} \theta \frac{\partial^{2} p_{0}}{\partial \varphi^{2}}+\frac{\cot \theta}{r^{2}} \sin ^{2} \theta \frac{\partial p_{0}}{\partial \theta}\right)+\frac{2 \mu \delta^{2}}{2}\left(\frac{\partial u_{1}^{1}}{\partial \theta}+\frac{\partial u_{1}^{2}}{\partial \varphi}+\cot \theta u_{1}^{1}\right)=F_{p}^{1}
\end{array}\right.
$$


In particular, if the flow is axial symmetric then

$$
\left\{\begin{array}{l}
-\frac{\mu \delta^{3}}{3}\left[\frac{2}{r^{2}} \frac{\partial^{2} u_{1}^{1}}{\partial \theta^{2}}+\frac{2 \cot \theta}{r^{2}} \frac{\partial u_{1}^{1}}{\partial \theta}-2 \cot ^{2} \theta u_{1}^{1}\right]+\frac{\mu}{2}\left(\delta-\frac{4}{r} \frac{\delta^{2}}{2}+\frac{6}{r^{2}} \frac{\delta^{3}}{3}\right) u_{1}^{1} \\
+\frac{1}{r^{2}}\left(\frac{3}{2} \frac{\delta^{2}}{2}-\frac{4}{r} \frac{\delta^{3}}{3}\right) \frac{\partial p_{0}}{\partial \theta}-\frac{\delta^{4}}{4}\left[\frac{1}{2} \sin 2 \theta u_{1}^{2} u_{1}^{2}+\cot \theta u_{1}^{1} u_{1}^{1}\right] \\
=\frac{1}{r^{2}} F_{1}^{1}+\frac{\delta^{3}}{3} \frac{1}{r^{2}} \frac{\partial p_{1}}{\partial \theta}+\mathscr{F}_{1}^{1}\left(\mathbf{u}_{2}\right), \\
-\frac{\mu \delta^{3}}{3}\left[\frac{1}{r^{2}} \frac{\partial^{2} u_{1}^{2}}{\partial \theta^{2}}+\frac{\cot \theta}{r^{2}} \sin ^{2} \theta \frac{\partial u_{1}^{1}}{\partial \theta}+\frac{3 \cot \theta}{r^{2}} \frac{\partial u_{1}^{2}}{\partial \theta}\right]+\frac{\mu}{2} \sin ^{2} \theta\left(\delta-\frac{4}{r} \frac{\delta^{2}}{2}+\frac{6}{r^{2}} \frac{\delta^{3}}{3}\right) u_{1}^{2} \\
+\frac{\delta^{4}}{4}\left(u_{1}^{1} \frac{\partial u_{1}^{2}}{\partial \theta}-u_{1}^{2} \frac{\partial u_{1}^{1}}{\partial \theta}+\cot \theta u_{1}^{1} u_{1}^{2}\right)=\frac{1}{r^{2}} \sin ^{2} \theta F_{2}^{1}+\mathscr{F}_{2}^{1}\left(\mathbf{u}_{2}\right), \\
\quad-\frac{\delta^{3}}{3}\left(\frac{1}{r^{2}} \frac{\partial^{2} p_{0}}{\partial \theta^{2}}+\frac{\cot \theta}{r^{2}} \sin ^{2} \theta \frac{\partial p_{0}}{\partial \theta}\right)+\frac{\mu \delta^{2}}{2}\left(\frac{\partial u_{1}^{1}}{\partial \theta}+\cot \theta u_{1}^{1}\right)=F_{p}^{1}, \\
\left.\left(u_{1}^{\alpha}, p_{0}\right)\right|_{\theta=0 \cup \theta=\pi} ^{<\infty,}
\end{array}\right.
$$

where

$$
\begin{aligned}
& \mathscr{F}_{1}^{1}\left(\mathbf{u}_{2}\right)=\frac{1}{r^{2}}\left(\frac{3}{2} \delta^{-1}-\frac{1}{r}\right) F_{1}^{2}+\frac{\mu}{2} \frac{\delta^{3}}{3} \frac{1}{r^{2}} \partial_{\theta} u_{2}^{3}, \\
& \mathscr{F}_{2}^{1}\left(\mathbf{u}_{2}\right)=\frac{1}{r^{2}}\left(\frac{3}{2} \delta^{-1}-\frac{1}{r}\right) F_{2}^{2} .
\end{aligned}
$$

(5.7) is a two points boundary value problem for ordinary differential equations.

$$
\begin{aligned}
& \left\{\begin{array}{l}
u_{1}^{3}=0, \\
\frac{2 \mu \delta^{3}}{3} u_{2}^{1}=\frac{1}{r^{2}} F_{1}^{2}-\frac{\mu}{2} \frac{\delta^{2}}{2} u_{1}^{1}+\frac{1}{r^{2}} \partial_{\theta} p_{0},
\end{array}\right. \\
& \frac{2 \mu \delta^{3}}{3} u_{2}^{2}=\frac{1}{r^{2}} \sin ^{2} \theta F_{2}^{2}+\frac{\mu}{2} \frac{\delta^{2}}{2} u_{1}^{2} \\
& \left\{u_{2}^{3}=-\frac{1}{2}\left(\partial_{\theta} u_{1}^{1}+\cot \theta u_{1}^{1}\right)\right. \text {, } \\
& \frac{\delta^{3}}{3} p_{1}=\frac{1}{2} F_{3}^{2}+\left(\frac{1}{r} \frac{\delta \delta^{3}}{3}-\frac{\delta^{2}}{2}\right) p_{0}+\frac{5 \mu \delta^{3}}{3}\left(\partial_{\theta} u_{1}^{1}+\cot \theta u_{1}^{1}\right), \\
& \frac{\delta^{3}}{3} p_{2}=\left(\frac{4}{r} \frac{\delta^{3}}{3}-\frac{\delta^{2}}{2}\right) p_{1}-\left(\delta-\frac{4}{r} \frac{\delta^{2}}{2}+\frac{3}{r^{2}} \frac{\delta^{3}}{3}\right) p_{0}+\frac{\delta^{4}}{4}\left(r u_{1}^{1} u_{1}^{1}+r \sin ^{2} \theta u_{1}^{2} u_{1}^{2}\right) \\
& -\frac{2 \mu \delta^{3}}{3} \frac{1}{r}\left(\partial_{\theta} u_{1}^{1}+\cot \theta u_{1}^{1}\right) \\
& \left\{\begin{array}{l}
h_{t}^{1}=\left.\mu\left(u_{1}^{1}+\frac{1}{r^{2}} \partial_{\theta} u_{0}^{3}-\frac{1}{r} u_{0}^{1}\right)\right|_{\mathfrak{I}(\delta)}, \quad h_{t}^{2}=\left.\mu\left(u_{1}^{2}+\frac{1}{r^{2}} \sin ^{2} \theta \partial_{\theta} u_{0}^{3}-\frac{1}{r} u_{0}^{2}\right)\right|_{\mathfrak{I}(\delta)}, \\
h_{t}^{3}=\left.\left(-p_{0}+2 \mu u_{1}^{3}\right)\right|_{\mathfrak{I}(\delta)}, \\
F_{1}^{1}=\delta r^{2} h_{t}^{1}, \quad F_{2}^{1}=\delta r^{2} \sin ^{2} \theta h_{t}^{2}, \quad F_{3}^{1}=\delta h_{t}^{3}, \quad F_{1}^{2}=\delta^{2} r^{2} h_{t}^{1}, \\
F_{2}^{2}=\delta^{2} r^{2} \sin ^{2} \theta h_{t}^{2}, \quad F_{p}=\delta^{2} r^{2}\left[\partial_{\theta} h_{t}^{1}+\sin ^{2} \theta \partial_{\varphi} h_{t}^{2}+\cot \theta h_{t}^{1}\right],
\end{array}\right.
\end{aligned}
$$

(B) SLE II

The first, we note 


$$
\left\{\begin{array}{l}
e_{11}^{*}(\mathbf{u})=r^{2} \frac{\partial u^{1}}{\partial \theta}, \\
*_{12}(\mathbf{u})=\frac{1}{2} r^{2}\left(\frac{\partial u^{1}}{\partial \varphi}+\sin \theta \frac{\partial u^{2}}{\partial \theta}\right), \\
*_{22}(\mathbf{u})=r^{2} \sin \theta \frac{\partial u^{2}}{\partial \varphi}+r^{2} \cos \theta u^{1}, \\
\gamma_{11}(\mathbf{u})=r^{2} \frac{\partial u^{1}}{\partial \theta}-r u_{0}^{3}, \\
\gamma_{12}(\mathbf{u})=\gamma_{21}(\mathbf{u})=\frac{1}{2} r^{2}\left(\frac{\partial u^{1}}{\partial \varphi}+\sin \theta \frac{\partial u^{2}}{\partial \theta}\right), \\
\gamma_{22}(\mathbf{u})=r^{2} \sin \theta \frac{\partial u^{2}}{\partial \varphi}+r^{2} \cos \theta u^{1}-u_{0}^{3} r \sin ^{2} \theta,
\end{array}\right.
$$

So that

$$
\begin{aligned}
& b^{\alpha \beta} \gamma_{\alpha \beta}\left(\mathbf{u}_{0}\right)=r\left(\frac{\partial u_{0}^{1}}{\partial \theta}+\frac{\partial u_{0}^{2}}{\partial \varphi}\right)+r \frac{\cot \theta}{\sin \theta} u_{0}^{1}+\frac{2}{r^{2}} u_{0}^{3}, \\
& \gamma_{0}\left(\mathbf{u}_{0}\right)=a^{\alpha \beta} \gamma_{\alpha \beta}\left(\mathbf{u}_{0}\right)=\frac{\partial u_{0}^{1}}{\partial \theta}+\frac{1}{\sin \theta} \frac{\partial u_{0}^{2}}{\partial \varphi}+\frac{\cot \theta}{\sin \theta} u_{0}^{1}-\frac{2}{r} u_{0}^{3}, \\
& \mathscr{S}_{3}^{0}\left(\mathbf{u}_{0}, \mathbf{u}_{0}\right)=u_{0}^{1} \frac{\partial u_{0}^{3}}{\partial \theta}+u_{0}^{2} \frac{\partial u_{0}^{3}}{\partial \varphi}+r u_{0}^{1} u_{0}^{1}+r \sin ^{2} \theta u_{0}^{2} u_{0}^{2},
\end{aligned}
$$

Taking (5.7) into account, we claim that

$$
\left\{\begin{array}{l}
-\mu \delta\left[\frac{2}{r^{2}}\left(\frac{\partial^{2} u_{0}^{1}}{\partial \theta^{2}}+\frac{\partial^{2} u_{0}^{2}}{\partial \theta \partial \varphi}\right)+\frac{1}{r^{2}} \sin ^{2} \theta \frac{\partial^{2} u_{0}^{1}}{\partial \theta \partial \varphi}+\frac{2 \cot \theta}{r^{2}}\left(\frac{\partial u_{0}^{1}}{\partial \theta}-\frac{\partial u_{0}^{2}}{\partial \varphi}\right)-2 \cot ^{2} \theta u_{0}^{1}+\frac{2}{r^{3}} \frac{\partial u_{0}^{3}}{\partial \theta}\right] \\
-\frac{\delta}{r^{2}} \frac{\partial p_{0}}{\partial \theta}+\delta\left[u_{0}^{2} \frac{\partial u_{0}^{1}}{\partial \varphi}-u_{0}^{1} \frac{\partial u_{0}^{2}}{\partial \varphi}-\frac{1}{2} \sin 2 \theta u_{0}^{2} u_{0}^{2}-\cot \theta u_{0}^{1} u_{0}^{1}\right]+\delta u_{1}^{1} u_{0}^{3}=\frac{1}{r^{2}} F_{1}^{0}, \\
-\mu \delta\left[\frac{1}{r^{2}} \frac{\partial^{2} u_{0}^{2}}{\partial \theta^{2}}+\frac{1}{r^{2}} \sin ^{2} \theta \frac{\partial^{2} u_{0}^{2}}{\partial \varphi^{2}}+\frac{\cot \theta}{r^{2}} \sin ^{2} \theta\left(\frac{\partial u_{0}^{1}}{\partial \theta}+\frac{\partial u_{0}^{1}}{\partial \varphi}\right)+\frac{3 \cot \theta}{r^{2}} \frac{\partial u_{0}^{2}}{\partial \theta}+\frac{\cot \theta}{r^{2}} \sin ^{2} \theta \frac{\partial u_{0}^{2}}{\partial \varphi}+\frac{1}{r^{3}} \sin ^{2} \theta \theta_{\varphi} u_{0}^{3}\right] \\
-\frac{\delta}{r^{2}} \sin ^{2} \theta \frac{\partial p_{0}}{\partial \varphi}+\delta\left(u_{0}^{1} \frac{\partial u_{0}^{2}}{\partial \theta}-u_{0}^{2} \frac{\partial u_{0}^{1}}{\partial \theta}+\cot \theta u_{0}^{2} u_{0}^{1}+u_{0}^{3} u_{1}^{2}\right)=\frac{1}{r^{2}} \sin ^{2} \theta F_{2}^{0}, \\
-2 \mu \delta\left[\frac{1}{r^{2}} \frac{\partial^{2} u_{0}^{3}}{\partial \theta^{2}}+\frac{1}{r^{2}} \sin ^{2} \theta \frac{\partial^{2} u_{0}^{3}}{\partial \varphi^{2}}+\frac{\cot \theta}{r^{2}} \sin ^{2} \theta \frac{\partial u_{0}^{3}}{\partial \theta}\right]-2 \mu \delta\left[r\left(\frac{\partial u_{0}^{1}}{\partial \theta}+\frac{\partial u_{0}^{2}}{\partial \varphi}\right)+r \frac{\cot \theta}{\sin \theta} u_{0}^{1}+\frac{2}{r^{2}} u_{0}^{3}\right] \\
+\delta\left[u_{0}^{1} \frac{\partial u_{0}^{3}}{\partial \theta}+u_{0}^{2} \frac{\partial u_{0}^{3}}{\partial \varphi}+r u_{0}^{1} u_{0}^{1}+r \sin ^{2} \theta u_{0}^{2} u_{0}^{2}\right]-\frac{2}{r} p_{0}=2 \mu \delta\left(\partial_{\theta} u_{1}^{1}+\partial_{\varphi} u_{1}^{2}+\cot \theta u_{1}^{1}\right)+F_{3}^{0}, \\
-\frac{\delta^{2}}{2}\left(\frac{1}{r^{2}} \frac{\partial^{2} p_{0}}{\partial \theta^{2}}+\frac{1}{r^{2}} \sin ^{2} \theta \frac{\partial^{2} p_{0}}{\partial \varphi^{2}}+\frac{\cot \theta}{r^{2}} \sin ^{2} \theta \frac{\partial p_{0}}{\partial \theta}\right)=-2 \mu \delta\left[\partial_{\theta} u_{0}^{1}+\partial_{\varphi} u_{0}^{2}+\cot \theta u_{0}^{1}\right]+F_{p}^{1},
\end{array}\right.
$$

If the flow is symmetric then 


$$
\left\{\begin{array}{l}
-\mu \delta\left[\frac{2}{r^{2}}\left(\frac{\partial^{2} u_{0}^{1}}{\partial \theta^{2}}+\frac{1}{r^{2}}\left(2-\frac{\cot \theta}{\sin ^{2} \theta}\right) u_{0}^{1}+\frac{2 \cot \theta}{r^{2}}\left(\frac{\partial u_{0}^{1}}{\partial \theta}\right)\right)+\frac{1}{r^{3}} \partial_{\theta} u_{0}^{3}\right] \\
-\frac{1}{r^{2}} \delta \frac{\partial p_{0}}{\partial \theta}+\delta\left[-\frac{1}{2} \sin 2 \theta u_{0}^{2} u_{0}^{2}-\cot \theta u_{0}^{1} u_{0}^{1}\right]+\delta u_{1}^{3} u_{0}^{3}=\frac{1}{r^{2}} F_{1}^{0}, \\
-\mu \delta\left[\frac{1}{r^{2}} \frac{\partial^{2} u_{0}^{2}}{\partial \theta^{2}}+\frac{\cot \theta}{r^{2}} \sin ^{2} \theta\left(\frac{\partial u_{0}^{1}}{\partial \theta}\right)+\frac{3 \cot \theta}{r^{2}} \frac{\partial u_{0}^{2}}{\partial \theta}\right]+\delta\left(u_{0}^{1} \frac{\partial u_{0}^{2}}{\partial \theta}-u_{0}^{2} \frac{\partial u_{0}^{1}}{\partial \theta}+\cot \theta u_{0}^{2} u_{0}^{1}+u_{0}^{3} u_{1}^{2}\right)=\frac{1}{r^{2}} \sin ^{2} \theta F_{2}^{0}, \\
-2 \mu \delta\left[\frac{1}{r^{2}} \frac{\partial^{2} u_{0}^{3}}{\partial \theta^{2}}+\frac{\cot \theta}{r^{2}} \sin ^{2} \theta \frac{\partial u_{0}^{3}}{\partial \theta}\right]-2 \mu \delta\left[r \frac{\partial u_{0}^{1}}{\partial \theta}+r \frac{\cot \theta}{\sin \theta} u_{0}^{1}+\frac{2}{r^{2}} u_{0}^{3}\right] \\
+\delta\left[u_{0}^{1} \frac{\partial u_{0}^{3}}{\partial \theta}+r u_{0}^{1} u_{0}^{1}+r \sin ^{2} \theta u_{0}^{2} u_{0}^{2}\right]-\frac{2}{r} p_{0}=2 \mu \delta\left(\partial_{\theta} u_{1}^{1}+\cot \theta u_{1}^{1}\right)+F_{3}^{0}, \\
-\frac{\delta^{2}}{2}\left(\frac{1}{r^{2}} \frac{\partial^{2} p_{0}}{\partial \theta^{2}}+\frac{\cot \theta}{r^{2}} \sin ^{2} \theta \frac{\partial p_{0}}{\partial \theta}\right)=-2 \mu \delta\left[\partial_{\theta} u_{0}^{1}+\cot \theta u_{0}^{1}\right]+F_{p}^{1},
\end{array}\right.
$$

and

$$
\left\{\begin{aligned}
& \mu \delta u_{1}^{1}= r^{-2} F_{1}^{1}-\frac{\delta^{2}}{2}(r)^{-2} \partial_{\theta} p_{0}+\frac{\delta^{2}}{2}\left[u_{0}^{1} \partial_{\theta} u_{0}^{1}+u_{0}^{2} \partial_{\varphi} u_{0}^{1}-\frac{1}{2} \sin 2 \theta u_{0}^{2} u_{0}^{2}-\frac{2}{r} u_{0}^{1} u_{0}^{3}\right] \\
&+\frac{\mu \delta^{2}}{2}\left[\frac{2}{r^{2}}\left(\frac{\partial^{2} u_{0}^{1}}{\partial \theta^{2}}+\frac{\partial^{2} u_{0}^{2}}{\partial \theta \partial \varphi}\right)+\frac{1}{r^{2}} \sin ^{2} \theta \frac{\partial^{2} u_{0}^{1}}{\partial \theta \partial \varphi}+\frac{2 \cot \theta}{r^{2}}\left(\frac{\partial u_{0}^{1}}{\partial \theta}-\frac{\partial u_{0}^{2}}{\partial \varphi}\right)-2 \cot ^{2} \theta u_{0}^{1}+\frac{2}{r^{3}} \frac{\partial u_{0}^{3}}{\partial \theta}\right], \\
& \mu \delta u_{1}^{2}=(r \sin \theta)^{-2} F_{2}^{1}-\frac{\delta^{2}}{2}(r \sin \theta)^{-2} \partial_{\varphi} p_{0}+\frac{\delta^{2}}{2}\left[u_{0}^{1} \partial_{\theta} u_{0}^{2}+u_{0}^{2} \partial_{\varphi} u_{0}^{2}+2 \cot \theta u_{0}^{1} u_{0}^{2}-\frac{2}{r} u_{0}^{2} u_{0}^{3}\right] \\
&+ \frac{\mu \delta^{2}}{2}\left[\frac{1}{r^{2}} \frac{\partial^{2} u_{0}^{2}}{\partial \theta^{2}}+\frac{1}{r^{2}} \sin ^{2} \theta \frac{\partial^{2} u_{0}^{2}}{\partial \varphi^{2}}+\frac{\cot \theta}{r^{2}} \sin ^{2} \theta\left(\frac{\partial u_{0}^{1}}{\partial \theta}+\frac{\partial u_{0}^{1}}{\partial \varphi}\right)+\frac{3 \cot \theta}{r^{2}} \frac{\partial u_{0}^{2}}{\partial \theta}+\frac{\cot \theta}{r^{2}} \sin ^{2} \theta \frac{\partial u_{0}^{2}}{\partial \varphi}+\frac{1}{r^{3}} \sin ^{2} \theta \partial_{\varphi} u_{0}^{3}\right] \\
& u_{1}^{3}+\partial_{\theta} u_{0}^{1}+\partial_{\varphi} u_{0}^{2}+\cot \theta u_{0}^{1}-\frac{2}{r} u_{0}^{3}=0, \\
& \frac{\delta^{2}}{2} p_{1}= F_{3}^{1}-\left(\delta-\frac{4}{r} \frac{\delta^{2}}{2}\right) p_{0}-2 \mu \delta u_{1}^{3}+\frac{\mu}{2} \frac{\delta^{2}}{2}\left[u_{0}^{1} \partial_{\theta} u_{0}^{3}+u_{0}^{2} \partial_{\varphi} u_{0}^{3}+r u_{0}^{1} u_{0}^{1}+r \sin ^{2} \theta u_{0}^{2} u_{0}^{2}\right] \\
&+\frac{\mu \delta^{2}}{2}\left[\frac{1}{r^{2}} \frac{\partial^{2} u_{0}^{3}}{\partial \theta^{2}}+\frac{1}{r^{2}} \sin ^{2} \theta \frac{\partial^{2} u_{0}^{3}}{\partial \varphi^{2}}+\frac{\cot \theta}{r^{2}} \sin ^{2} \theta \frac{\partial u_{0}^{3}}{\partial \theta}\right],
\end{aligned}\right.
$$

where

$$
\begin{aligned}
& F_{1}^{0}=r^{2}\left(h_{t}^{1}-h_{b}^{1}\right), \quad F_{2}^{0}=r^{2} \sin ^{2} \theta\left(h_{t}^{2}-h_{b}^{2}\right), \quad F_{3}^{0}=h_{t}^{3}-h_{b}^{3}, \\
& h_{t}^{1}=\mu\left(u_{1}^{1}+r^{-2} \partial_{\theta} u_{0}^{3}-r^{-1} u_{0}^{1}\right)(k), \quad h_{b}^{1}=\mu\left(u_{1}^{1}+r^{-2} \partial_{\theta} u_{0}^{3}-r^{-1} u_{0}^{1}\right)(k-1), \\
& h_{t}^{2}=\mu\left(u_{1}^{2}-r^{-1} u_{0}^{1}\right)(k), \quad h_{b}^{2}=\mu\left(u_{1}^{2}-r^{-1} u_{0}^{1}\right)(k-1), \\
& h_{t}^{3}=\left(-p_{0}+2 \mu u_{1}^{3}\right)(k), \quad h_{b}^{3}=\left(-p_{0}+2 \mu u_{1}^{3}\right)(k-1), \\
& F_{p}^{1}=\left.\delta \mu\left[\partial_{\theta} u_{1}^{1}+\cot \theta u_{1}^{1}+\frac{1}{r} \partial_{\theta} u_{0}^{1}+\frac{1}{r} \cot \theta u_{0}^{1}+\frac{1}{r^{2}} \partial_{\theta}^{2} u_{0}^{3}+\frac{\cot \theta}{r^{2}} \sin ^{2} \theta \partial_{\theta} u_{0}^{3}\right]\right|_{k},
\end{aligned}
$$

The drag is given by

$$
\begin{aligned}
& F_{d}=\int_{\mathfrak{I}}\left(-p_{0} \frac{x_{1} y_{2}-x_{2} y_{1}}{\sqrt{a}}+\mu u_{1}^{\alpha} z_{\alpha}\right) \sqrt{a} \mathrm{~d} x=-\int_{\mathfrak{I}}\left(p_{0} \cos \theta+\mu \gamma u_{1}^{1} \sin \theta\right) r^{2} \sin \theta \mathrm{d} \theta \mathrm{d} \varphi, \\
& F_{d}=\int_{\mathfrak{I}}\left(-p_{0} \cos \theta+\mu r u_{1}^{1} \sin \theta\right) r^{2} \sin \theta \mathrm{d} \theta \mathrm{d} \varphi .
\end{aligned}
$$




\subsection{The Flow around an Ellipsoid}

Let parametric equation of the ellipsoid be given by

$$
\left\{\begin{array}{l}
\mathbf{r}=x \mathbf{i}+y \mathbf{j}+z \mathbf{k}, \\
x=\alpha \cos \varphi \sin \theta, \quad y=\beta \sin \varphi \sin \theta, \quad z=\gamma \cos \theta \\
0<\gamma<\beta<\alpha, \quad \alpha, \beta, \gamma=\text { constants, }
\end{array}\right.
$$

where $(\mathbf{i}, \mathbf{j}, \mathbf{k})$ are Cartesian basis, $(\varphi, \theta)$ are the parameters and $\left(x^{2}=\varphi, x^{1}=\theta\right)$ are called Guassian coordinates of ellipsoid. The base vectors on the ellipsoid

$$
\begin{aligned}
& \mathbf{e}_{1}=\partial_{\theta} \mathbf{r}=\alpha \cos \varphi \cos \theta \mathbf{i}+\beta \sin \varphi \cos \theta \mathbf{j}-\gamma \sin \theta \mathbf{k}, \\
& \mathbf{e}_{2}=\partial_{\varphi} \mathbf{r}=-\alpha \sin \varphi \sin \theta \mathbf{i}+\beta \cos \varphi \sin \theta \mathbf{j}, \\
& n=\frac{1}{\sqrt{a}} \mathbf{e}_{1} \times \mathbf{e}_{2}=\frac{1}{\sqrt{a}}\left[-\beta \gamma \cos \varphi \sin ^{2} \theta \mathbf{i}+\alpha \gamma \sin \varphi \sin ^{2} \theta \mathbf{j}+\alpha \beta \sin \theta \cos \theta \mathbf{k}\right],
\end{aligned}
$$

The metric tensor of the ellipsoid is given by

$$
\left\{\begin{array}{l}
a_{\alpha \beta}=\mathbf{e}_{\alpha} \mathbf{e}_{\beta}, \\
a_{22}=\Lambda(\varphi) \sin ^{2} \theta, \quad a_{11}=\Lambda(\varphi) \cos ^{2} \theta+\gamma^{2} \sin ^{2} \theta \\
a_{12}=0, \quad \Lambda(\varphi)=\alpha^{2} \sin ^{2} \varphi+\beta^{2} \cos ^{2} \varphi \\
a=\operatorname{det}\left(a_{\alpha \beta}\right)=a_{0} \sin ^{2} \theta, \quad a_{0}=\Lambda(\varphi)\left[\Lambda(\varphi) \cos ^{2} \theta+\gamma^{2} \sin ^{2} \theta\right] \\
a^{22}=a_{11} / a=a_{22}^{-1}, \quad a^{11}=a_{22} / a=a_{11}^{-1}, \quad a^{12}=a^{21}=0
\end{array}\right.
$$

Curvature tensor, mean curvature and Gaussian curvature are given by

$$
\left\{\begin{array}{l}
b_{11}=b_{22}=\frac{\alpha \beta \gamma \sin ^{2} \theta}{\sqrt{a_{0}}}, \quad b_{12}=0, \quad b=\operatorname{det}\left(b_{\alpha \beta}\right)=\frac{\alpha^{2} \beta^{2} \gamma^{2} \sin ^{4} \theta}{a_{0}} \\
b^{11}=\frac{\alpha \beta \gamma}{\sqrt{a_{0}}} \frac{\sin ^{2} \theta}{a_{11} a_{11}}, \quad b^{22}=\frac{\alpha \beta \gamma}{\sqrt{a_{0}}} \frac{\sin ^{2} \theta}{a_{22} a_{22}} \\
b^{12}=b^{21}=0, \quad b_{1}^{1}=\frac{\alpha \beta \gamma}{\sqrt{a_{0}}} \frac{\sin ^{2} \theta}{a_{11}}, \quad b_{2}^{2}=\frac{\alpha \beta \gamma}{\sqrt{a_{0}}} \frac{\sin ^{2} \theta}{a_{22}} \\
K=\frac{b}{a}=\frac{(\alpha \beta \gamma)^{2} \sin ^{2} \theta}{a_{0}^{2}}, \quad H=\frac{\alpha \beta \gamma}{\sqrt{a_{0}}} \frac{\Lambda(\varphi)+\gamma^{2} \sin ^{2} \theta}{a_{0}}
\end{array}\right.
$$

\section{Semi-Geodesic Coordinate System Based on Ellipsoid $\mathfrak{I}$}

That is

$$
x^{2}=\varphi, \quad x^{1}=\theta, \quad x^{3}=\xi
$$

The radial vector at any point in $\mathfrak{R}^{3}$

$$
\begin{gathered}
\mathbf{R}=\mathbf{r}+\xi \mathbf{n} \\
\mathbf{n}=\mathbf{e}_{1} \times \mathbf{e}_{2} / \sqrt{\left|\mathbf{e}_{1} \times \mathbf{e}_{2}\right|}=\frac{\mathbf{e}_{1} \times \mathbf{e}_{2}}{\sqrt{a_{0}}}=\frac{1}{2} \varepsilon^{\alpha \beta} \mathbf{e}_{\alpha} \times \mathbf{e}_{\beta}
\end{gathered}
$$

Corresponding metric tensor of $\mathfrak{R}^{3}$ are given by (2.1). We remainder to give the covariant derivatives of the velocity field, Laplace-Betrami operator and trace-Laplace operator. To do this we have to give the first and second kind of Christoffel symbols on the ellipsoid $\mathfrak{I}$ as a two dimensional manifolds

$$
\begin{aligned}
& \Gamma_{11}^{*}=\frac{1}{2} \frac{\left(\gamma^{2}-\Lambda(\varphi)\right)}{a_{11}} \sin 2 \theta, \quad \Gamma_{12}^{*}=\frac{\alpha^{2}-\beta^{2}}{2} \frac{\cos ^{2} \theta}{a_{11}} \sin 2 \varphi, \quad \Gamma^{2}{ }_{11}=-\frac{\alpha^{2}-\beta^{2}}{2} \sin 2 \varphi \frac{\cos ^{2} \theta}{a_{22}}, \\
& \Gamma^{*}{ }_{12}=\cot \theta, \quad \Gamma^{*}{ }_{22}=\frac{\alpha^{2}-\beta^{2}}{2} \sin 2 \varphi \frac{\sin ^{2} \theta}{a_{22}}, \quad \Gamma^{*}{ }_{22}=-2 \frac{a_{22}}{a_{11}} \cot \theta,
\end{aligned}
$$


Then covariant derivatives of vector $\mathbf{u}=u^{\alpha} \mathbf{e}_{\alpha}+u^{3} \mathbf{n}$ on the two dimensional manifold $\mathfrak{I}$

$$
\begin{aligned}
& \stackrel{*}{\nabla} u^{1}=\partial_{\theta} u^{1}+\frac{1}{2} \sin 2 \theta \frac{\left(\gamma^{2}-\Lambda(\varphi)\right)}{a_{11}} u^{1}+\frac{\alpha^{2}-\beta^{2}}{2} \frac{\cos ^{2} \theta}{a_{11}} \sin 2 \varphi u^{2}, \\
& \stackrel{*}{\nabla}_{2} u^{1}=\partial_{\varphi} u^{1}+\frac{1}{a_{11}}\left[\frac{\alpha^{2}-\beta^{2}}{2} \cos ^{2} \theta \sin 2 \varphi u^{1}-\Lambda(\varphi) \sin 2 \theta u^{2}\right], \\
& \stackrel{*}{\nabla}_{1} u^{2}=\partial_{\theta} u^{2}+\left[\frac{\beta^{2}-\alpha^{2}}{2} \sin 2 \varphi \frac{\cos ^{2} \theta}{a_{22}} u^{1}+\cot \theta u^{2}\right], \stackrel{*}{\nabla}_{2} u^{2}=\partial_{\varphi} u^{2}+\cot \theta u^{1}+\frac{\alpha^{2}-\beta^{2}}{2} \frac{\sin ^{2} \theta}{a_{22}} \sin 2 \varphi u^{2}, \\
& \operatorname{div}^{*} \mathbf{u}=\partial_{\theta} u^{1}+\partial_{\varphi} u^{2}+d_{1} u^{1}+d_{2} u^{2}, d_{1}=\frac{1}{2} \frac{\left(\gamma^{2}-\Lambda(\varphi)\right)}{a_{11}} \sin 2 \theta+\cot \theta, \\
& d_{2}=\frac{\alpha^{2}-\beta^{2}}{2} \sin 2 \varphi\left[\frac{\cos ^{2} \theta}{a_{11}}+\frac{\sin ^{2} \theta}{a_{22}}\right],
\end{aligned}
$$

The associated Laplace-Betrami operator on $\mathfrak{I}$

$$
\begin{aligned}
& \stackrel{*}{\Delta} p_{0}=\frac{1}{a_{11}} \frac{\partial^{2} p_{0}}{\partial \theta^{2}}+\frac{1}{a_{22}} \frac{\partial^{2} p_{0}}{\partial \varphi^{2}}+C_{1} \frac{\partial p_{0}}{\partial \theta}+C_{2} \frac{\partial p_{0}}{\partial \varphi} \\
& C_{1}:=\frac{\alpha^{2}-\beta^{2}}{2 a_{11}^{2} \Lambda(\varphi)}\left(a_{11} \sin 2 \varphi+2 \Lambda(\varphi) \cos ^{2} \theta\left(1-\ln \sqrt{a_{11}}\right)\right)-\frac{\Lambda \ln \Lambda(\varphi)}{2 a_{11}^{2}} \sin 2 \theta \\
& C_{2}:=\frac{\alpha^{2}-\beta^{2}}{2 a_{11} \Lambda^{2}(\varphi)}\left(\Lambda(\varphi)-\gamma^{2}\right) \sin 2 \varphi
\end{aligned}
$$

Trace-Laplace operatoe on $\mathfrak{I}$

$$
\begin{aligned}
& \dot{\Delta}^{*} u^{\alpha}=a^{\lambda \sigma} \frac{\partial^{2} u^{\alpha}}{\partial x^{\lambda} \partial x^{\sigma}}+A_{\mu}^{\alpha \tau} \frac{\partial u^{\mu}}{\partial x^{\tau}}+A_{\mu}^{\alpha} u^{\mu}, \\
& A_{1}^{1}=\frac{\gamma^{2}-\Lambda(\varphi)}{a_{11}^{3}} \cos ^{2} \theta\left(\Lambda(\varphi) \cos ^{2} \theta+\gamma^{2}\left(1-3 \sin ^{2} \theta\right)\right) \\
& +\frac{\alpha^{2}-\beta^{2}}{2 a_{11}^{2} a_{22} \Lambda(\varphi)}\left[\Lambda^{2}(\varphi) \sin 2 \varphi \cot \theta \cos ^{2} \theta-\left(\frac{\alpha^{2}+\beta^{2}}{8} \gamma^{2} \sin ^{2} 2 \varphi-\Lambda(\varphi) \sin ^{2} \theta\right) \sin ^{2} 2 \theta\right], \\
& A_{2}^{2}=\frac{\sin ^{3} \theta \cos \theta}{a_{11}^{2} a_{22}^{2}} \times\left[\frac{\alpha^{2}-\beta^{2}}{2} \Lambda(\varphi) \sin 2 \varphi\left(\Lambda(\varphi) \cos ^{2} \theta+\gamma^{2} \sin ^{2} \theta\right)-\frac{1}{2} \Lambda^{2}(\varphi)\left(\gamma^{2}-\Lambda\right) \sin 2 \theta\right. \\
& \left.-\frac{1}{2} \gamma^{2} \sin 2 \theta\left(\frac{\alpha^{2}-\beta^{2}}{2} \sin 2 \varphi\right)^{2}\right]-\frac{1}{a_{11} \sin ^{2} \theta}+\frac{\alpha^{2}-\beta^{2}}{a_{22}^{3}} \sin ^{4} \theta\left(\beta^{2} \cos ^{4} \varphi-\alpha^{2} \sin ^{4} \varphi\right), \\
& A_{2}^{1}=\frac{\alpha^{2}-\beta^{2}}{2} \frac{2 \sin 2 \varphi}{a_{11}^{3}}\left[2 \Lambda(\varphi) \cos ^{2} \theta \cot \theta\left(1-\frac{\alpha^{2}-\beta^{2}}{4} \sin 2 \varphi\right)-\gamma^{2} \sin 2 \theta \frac{\alpha^{2}-\beta^{2}}{4} \sin 2 \varphi\right] \\
& -\frac{\alpha^{2}-\beta^{2}}{a_{22} a_{11}^{2}} \sin ^{2} 2 \theta \sin ^{2} \theta \\
& A_{1}^{2}=\frac{1}{a_{11} a_{22}} \frac{\beta^{2}-\alpha^{2}}{4} \sin 2 \varphi \sin 2 \theta\left(1+(1+\cot \theta)^{2}\right), \\
& A_{1}^{11}=-\frac{1}{2 a_{11}^{2}}\left[\left(\gamma^{2}+\Lambda(\varphi)\right) \sin 2 \theta+4 \Lambda(\varphi) \cot \theta\right], \quad A_{2}^{11}=\frac{\alpha^{2}-\beta^{2}}{a_{11}^{2}} \cos ^{2} \theta \sin 2 \varphi, \\
& A_{1}^{12}=\frac{\left(\alpha^{2}-\beta^{2}\right) \sin 2 \varphi}{2 a_{11}^{2} a_{22}^{2}} \sin ^{2} \theta\left(2 \Lambda(\varphi) \cos ^{2} \theta-\gamma^{2} \sin ^{2} \theta\right),
\end{aligned}
$$




$$
\begin{aligned}
& A_{1}^{21}=\frac{2 \Gamma^{*}{ }_{11}}{a_{11}}=-\frac{\alpha^{2}-\beta^{2}}{a_{11} a_{22}} \sin 2 \varphi \cos ^{2} \theta, \quad A_{2}^{12}=\frac{2 \Gamma^{1}{ }_{22}}{a_{22}}=-4 \frac{\cot \theta}{a_{11}}, \\
& A_{2}^{22}=-\frac{\Gamma^{2}{ }_{11}}{a_{11}}+\frac{\Gamma^{2}{ }_{22}}{a_{22}}=\frac{\alpha^{2}-\beta^{2}}{2} \sin 2 \varphi\left(2 \cos ^{2} \theta+\left(\frac{\gamma}{\Lambda(\varphi)}\right)^{2} \sin ^{2} \theta\right),
\end{aligned}
$$

In addition, nonlinear terms

$$
\left\{\begin{array}{l}
B^{\alpha}\left(\mathbf{u}_{1}, \mathbf{u}_{1}\right):=u_{1}^{\lambda} \nabla_{\lambda}^{*} u_{1}^{\alpha}-u_{1}^{\alpha} \operatorname{div}_{1}^{*} \mathbf{u}_{1}=\operatorname{rot}^{\alpha} \mathbf{u}_{1}+d_{11}^{\alpha} u_{1}^{1} u_{1}^{1}+d_{12}^{\alpha} u_{1}^{1} u_{1}^{2}+d_{22}^{\alpha} u_{1}^{2} u_{1}^{2}, \\
B^{3}\left(\mathbf{u}_{1}, \mathbf{u}_{1}\right)=u_{1}^{\lambda} \nabla_{\lambda}^{*} u_{1}^{3}+b_{\alpha \beta} u_{1}^{\alpha} u_{1}^{\beta}=u_{1}^{1} \partial_{\theta} u_{1}^{3}+u_{1}^{2} \partial_{\varphi} u_{1}^{3}+b_{11}\left(u_{1}^{1} u_{1}^{1}+u_{1}^{2} u_{1}^{2}\right), \\
\operatorname{rot}^{1}\left(\mathbf{u}_{1}\right)=u_{1}^{2} \partial_{\varphi} u_{1}^{1}-u_{1}^{1} \partial_{\varphi} u_{1}^{2}, \quad \operatorname{rot}^{2}\left(\mathbf{u}_{1}\right)=u_{1}^{1} \partial_{\theta} u_{1}^{2}-u_{1}^{2} \partial_{\theta} u_{1}^{1}, \\
d_{11}^{1}=\frac{1}{2} \frac{\gamma^{2}-\Lambda(\varphi)}{a_{11}} \sin 2 \theta-d_{1}, \quad d_{22}^{1}=-\frac{\Lambda(\varphi)}{a_{11}} \sin 2 \theta \\
d_{12}^{1}=\left(\alpha^{2}-\beta^{2}\right) \sin 2 \varphi \frac{\cos ^{2} \theta}{a_{11}}-d_{2}, \\
d_{11}^{2}=-\frac{\alpha^{2}-\beta^{2}}{2} \sin 2 \varphi \frac{\cos ^{2} \theta}{a_{22}}, \quad d_{22}^{2}=\frac{\alpha^{2}-\beta^{2}}{2} \sin 2 \varphi \frac{\sin ^{2} \theta}{a_{22}}-d_{2}, \\
d_{12}^{2}=2 \cot \theta-d_{1},
\end{array}\right.
$$

and linear terms

$$
\left\{\begin{array}{l}
a^{11} \frac{\partial}{\partial \theta} \operatorname{div} \mathbf{u}_{1}=\frac{1}{a_{11}} \frac{\partial^{2} u_{1}^{1}}{\partial \theta^{2}}+\frac{1}{a_{11}} \frac{\partial^{2} u_{1}^{2}}{\partial \theta \partial \varphi}+\frac{d_{1}}{a_{11}} \frac{\partial u_{1}^{1}}{\partial \theta}+\frac{d_{2}}{a_{11}} \frac{\partial u_{1}^{2}}{\partial \theta}+\frac{\partial}{\partial \theta}\left(\frac{d_{1}}{a_{11}}\right) u_{1}^{1}+\frac{\partial}{\partial \theta}\left(\frac{d_{2}}{a_{11}}\right) u_{1}^{2}, \\
a^{22} \frac{\partial}{\partial \phi} \operatorname{div}_{1}^{*}=\frac{1}{a_{22}} \frac{\partial^{2} u_{1}^{1}}{\partial \theta \partial \varphi}+\frac{1}{a_{22}} \frac{\partial^{2} u_{1}^{2}}{\partial \varphi^{2}}+\frac{d_{1}}{a_{22}} \frac{\partial u_{1}^{1}}{\partial \varphi}+\frac{d_{2}}{a_{22}} \frac{\partial u_{1}^{2}}{\partial \varphi}+\frac{\partial}{\partial \varphi}\left(\frac{d_{1}}{a_{22}}\right) u_{1}^{1}+\frac{\partial}{\partial \varphi}\left(\frac{d_{2}}{a_{22}}\right) u_{1}^{2}, \\
\beta_{0}\left(\mathbf{u}_{1}\right)=b^{\alpha \beta} \gamma_{\alpha \beta}\left(\mathbf{u}_{1}\right)=\frac{\alpha \beta \gamma}{\sqrt{a_{0}}} \sin ^{2} \theta\left[\frac{1}{\Lambda^{2}(\varphi)} \stackrel{\nabla}{\nabla}_{1}^{*} u_{1}^{1}+\frac{\sin ^{2} \theta}{a_{11}} \stackrel{\nabla}{2}_{2}^{*} u_{1}^{2}\right]-\left(4 H^{2}-2 K\right) u_{1}^{3}, \\
\gamma_{0}\left(\mathbf{u}_{1}\right)=a^{\alpha \beta} \gamma_{\alpha \beta}\left(\mathbf{u}_{1}\right)=\operatorname{div} \mathbf{u}_{0}-2 H u_{1}^{3},
\end{array}\right.
$$

(i) BLE I. Taking (5.1-5.6) and above formula into account we obtain BLE I on the ellipsoid

$$
\left\{\begin{array}{c}
-\frac{\mu \delta^{3}}{3}\left[\frac{1}{a_{11}}\left(2 \frac{\partial^{2} u_{1}^{1}}{\partial \theta^{2}}+\frac{\partial^{2} u_{1}^{2}}{\partial \theta \partial \varphi}\right)+\frac{1}{a_{22}} \frac{\partial^{2} u_{1}^{1}}{\partial \varphi^{2}}+A_{\sigma}^{1 \lambda} \frac{\partial u_{1}^{\sigma}}{\partial x^{\lambda}}+\left(A_{\sigma}^{1}+K \delta_{\lambda}^{1}\right) u_{1}^{\lambda}+\frac{\partial}{\partial \theta}\left(\frac{d_{\lambda} u_{1}^{\lambda}}{a_{11}}\right)\right] \\
+\frac{Q_{11}}{a_{11}} u_{1}^{1}+\frac{\delta^{4}}{4} B^{1}\left(\mathbf{u}_{1}, \mathbf{u}_{1}\right)+d^{11} \partial_{\theta} p_{0}-\frac{4 \delta^{3}}{3} \frac{1}{a_{11}} \partial_{\theta}\left(H p_{0}\right)=\frac{1}{a_{11}}\left[\mathscr{T}_{1}^{1}-\frac{\delta^{3}}{3} \partial_{\theta} p_{1}\right] \\
-\frac{\mu \delta^{3}}{3}\left[\frac{1}{a_{11}} \frac{\partial^{2} u_{1}^{2}}{\partial \theta^{2}}+\frac{1}{a_{22}}\left(\frac{\partial^{2} u_{1}^{1}}{\partial \theta \partial \varphi}+2 \frac{\partial^{2} u_{1}^{2}}{\partial \varphi^{2}}\right)+A_{\sigma}^{2 \lambda} \frac{\partial u_{1}^{\sigma}}{\partial x^{\lambda}}+\left(A_{\sigma}^{2}+K \delta_{\lambda}^{2}\right) u_{1}^{\lambda}+\frac{\partial}{\partial \varphi}\left(\frac{d_{\lambda} u_{1}^{\lambda}}{a_{22}}\right)\right] \\
+\frac{Q_{22}}{a_{22}} u_{1}^{2}+\frac{\delta^{4}}{4} B^{2}\left(\mathbf{u}_{1}, \mathbf{u}_{1}\right)+d^{22} \partial_{\varphi} p_{0}-\frac{4 \delta^{3}}{3} \frac{1}{a_{22}} \partial_{\varphi}\left(H p_{0}\right)=\frac{1}{a_{22}}\left[\mathscr{F}_{2}^{1}-\frac{\delta^{3}}{3} \partial_{\varphi} p_{1}\right], \\
-\frac{\delta^{3}}{3}\left[a^{22} \frac{\partial^{2} p_{0}}{\partial \varphi^{2}}+a^{11} \frac{\partial^{2} p_{0}}{\partial \theta^{2}}+C_{2}(\varphi, \theta) \frac{\partial p_{0}}{\partial \varphi}+C_{1}(\varphi, \theta) \frac{\partial p_{0}}{\partial \theta}\right]+M_{0}\left(\mathbf{u}_{1}\right)=F_{p},
\end{array}\right.
$$

where 


$$
\left\{\begin{array}{l}
M_{0}\left(\mathbf{u}_{1}\right)=\{\operatorname{see}(5.5)\} m_{1}^{1} \partial_{\theta} u_{1}^{1}+m_{1}^{2} \partial_{\varphi} u_{2}^{2}+m_{0}^{\prime} u_{1}^{1}+m_{0}^{2} u_{1}^{2}, \\
m_{1}^{1}=\frac{\mu \delta^{2}}{2}-\frac{4 \mu \delta^{3}}{3 b_{2}^{2}}, \quad m_{1}^{2}=\frac{\mu \delta^{2}}{2}-\frac{4 \mu \delta^{3}}{3 b_{1}^{1}}, \\
m_{0}^{1}=\frac{\mu \delta^{2}}{2} d_{1}-\frac{4 \mu \delta^{3}}{3} \frac{\alpha \beta \gamma}{a_{0}^{3 / 2}}\left(\Lambda(\varphi) \sin ^{2} \theta-\left(\Lambda(\varphi)-\gamma^{2}\right) \cos ^{2} \theta\right)+\frac{6 \mu \delta^{3}}{3} \partial_{\theta} H, \\
m_{0}^{2}=\frac{\mu \delta^{2}}{2} d_{2}-\frac{4 \mu \delta^{3}}{3} \frac{\alpha \beta \gamma}{\sqrt{a_{0}}} \frac{\alpha^{2}-\beta^{2}}{2} \sin 2 \varphi\left(\sin ^{2} \theta+\cot ^{2} \theta \cos ^{2} \theta\right)+\frac{6 \mu \delta^{3}}{3} \partial_{\theta} H, \\
Q_{\alpha \alpha}=2 \mu\left\{\frac{\delta}{8}+\left(\frac{3}{2} H a_{\alpha \alpha}+b_{\alpha \alpha}\right) \frac{\delta^{2}}{2}+\left(\left(4 K-2 H^{2}\right) a_{\alpha \alpha}-4 H b_{\alpha \alpha}\right) \frac{\delta^{3}}{3}\right\},
\end{array}\right.
$$

$$
\left\{\begin{array}{l}
2 u_{2}^{3}+\partial_{\theta} u_{1}^{1}+\partial_{\varphi} u_{2}^{2}+d_{1} u_{1}^{1}+d_{2} u_{1}^{2}=0, \\
\frac{\delta^{3}}{3} p_{2}+\left(\frac{\delta^{2}}{2}-\frac{4 H \delta^{3}}{3}\right) p_{1}+(\delta-4 H+3 K) p_{0}-\frac{\delta^{4}}{4} \frac{\alpha \beta \gamma}{\sqrt{a_{0}}} \sin ^{2} \theta\left(u_{1}^{1} u_{1}^{1}+u_{1}^{2} u_{1}^{2}\right)+\frac{2 \mu \delta^{3}}{3} \beta_{0}\left(u_{1}\right)=-F_{3}^{1}, \\
\frac{2 \delta^{3}}{3} p_{1}+2\left(\frac{\delta^{2}}{2}-\frac{H \delta^{3}}{3}\right) p_{0}-\frac{5 \mu \delta^{3}}{3}\left(\partial_{\theta} u^{1}+\partial_{\varphi} u^{2}+d_{1} u_{1}^{1}+d_{2} u_{1}^{2}\right)=F_{3}^{2},
\end{array}\right.
$$

The right terms are given by

$$
\left\{\begin{array}{l}
a^{\alpha \beta} F_{\beta}^{1}=\delta h_{t}^{\alpha}, \quad F_{3}^{1}=\delta h_{t}^{3}, \quad a^{\alpha \beta} F_{\beta}^{2}=\delta^{2} h_{t}^{\alpha}, \quad F_{3}^{2}=\delta^{2} h_{t}^{3}, \\
h_{t}^{\alpha}=\left.\mu\left(u_{1}^{\alpha}+\frac{1}{a_{\alpha \alpha}} \nabla_{\alpha}^{*} u_{0}^{3}-H u_{0}^{\alpha}\right)\right|_{\mathfrak{I}_{1}}, \quad h_{t}^{3}=\left.\left(-p_{0}+2 \mu u_{1}^{3}\right)\right|_{\mathfrak{I}_{1}} .
\end{array}\right.
$$

(ii) SEL II. Let consider SEL II given by (5.7) and corresponding variational formulation (5.12) which are followings in semi geodesic coordinate system based on the ellipsoid

$$
\left\{\begin{aligned}
- & \mu \delta\left[\frac{1}{a_{11}}\left(2 \frac{\partial^{2} u_{0}^{1}}{\partial \theta^{2}}+\frac{\partial^{2} u_{0}^{2}}{\partial \theta \partial \varphi}\right)+\frac{1}{a_{22}} \frac{\partial^{2} u_{0}^{1}}{\partial \varphi^{2}}+A_{\sigma}^{1 \lambda} \frac{\partial u_{0}^{\sigma}}{\partial x^{\lambda}}+\left(A_{\sigma}^{1}+K \delta_{\lambda}^{1}\right) u_{0}^{\lambda}+\frac{\partial}{\partial \theta}\left(\frac{d_{\lambda} u_{0}^{\lambda}}{a_{11}}\right)+\frac{4}{a_{11}} \partial_{\theta} H u_{0}^{3}+2 b^{11} \partial_{\theta} u_{0}^{3}\right] \\
& +\delta \frac{1}{a_{11}} B^{1}\left(\mathbf{u}_{0}, \mathbf{u}_{0}\right)-\delta \frac{1}{a_{11}} \partial_{\theta} p_{0}=\frac{1}{a_{11}} F_{1}^{0}, \\
- & \frac{\mu \delta^{3}}{3}\left[\frac{1}{a_{11}} \frac{\partial^{2} u_{0}^{2}}{\partial \theta^{2}}+\frac{1}{a_{22}}\left(\frac{\partial^{2} u_{0}^{1}}{\partial \theta \partial \varphi}+2 \frac{\partial^{2} u_{0}^{2}}{\partial \varphi^{2}}\right)+A_{\sigma}^{2 \lambda} \frac{\partial u_{0}^{\sigma}}{\partial x^{\lambda}}+\left(A_{\sigma}^{2}+K \delta_{\lambda}^{2}\right) u_{0}^{\lambda}+\frac{\partial}{\partial \varphi}\left(\frac{d_{\lambda} u_{0}^{\lambda}}{a_{22}}\right)+\frac{4}{a_{22}} \partial_{\varphi} H u_{0}^{3}+2 b^{22} \partial_{\varphi} u_{0}^{3}\right] \\
& +\delta \frac{1}{a_{22}} B^{2}\left(\mathbf{u}_{0}, \mathbf{u}_{0}\right)-\delta \frac{1}{a_{22}} \partial_{\varphi} p_{0}=\frac{1}{a_{22}} F_{2}^{0}, \\
- & \mu \delta\left[-\frac{\delta^{3}}{3}\left[a^{22} \frac{\partial^{2} p_{0}}{\partial \varphi^{2}}+a^{11} \frac{\partial^{2} p_{0}}{\partial \theta^{2}}+C_{2}(\varphi, \theta) \frac{\partial p_{0}}{\partial \varphi}+C_{1}(\varphi, \theta) \frac{\partial p_{0}}{\partial \theta}\right]+\beta_{0}\left(\mathbf{u}_{0}\right)\right] \\
& +\delta B^{3}\left(\mathbf{u}_{0}, \mathbf{u}_{0}\right)-2 H \delta p_{0}-\mu \delta \operatorname{div} \mathbf{u}_{1}=F_{3}^{0},
\end{aligned}\right.
$$

where

$$
\beta_{0}\left(\mathbf{u}_{0}\right)=\frac{\alpha \beta \gamma}{\sqrt{a_{0}}} \sin ^{2} \theta\left[\frac{1}{\Lambda^{2}(\varphi)} \stackrel{*}{\nabla} u_{1}^{1}+\frac{\sin ^{2} \theta}{a_{11}}{ }^{*} \nabla_{2} u_{0}^{2}\right]-\left(4 H^{2}-2 K\right) u_{0}^{3}, \gamma_{0}\left(\mathbf{u}_{0}\right)=a^{\alpha \beta} \gamma_{\alpha \beta}\left(\mathbf{u}_{0}\right)=\stackrel{*}{\operatorname{div}} \mathbf{u}_{0}-2 H u_{0}^{3},
$$




$$
\begin{aligned}
& \left\{\begin{array}{l}
F_{\beta}^{0}=a_{\alpha \beta}\left(h_{t}^{\alpha}-h_{b}^{\alpha}\right), \quad F_{3}^{0}=h_{t}^{3}-h_{b}^{3}, \quad F_{\beta}^{1}=a_{\alpha \beta} h_{t}^{\alpha} \delta, \quad F_{3}^{1}=\delta h_{t}^{3}, \\
h_{b}^{\alpha}=\mu u_{1}^{\alpha}(k), \quad h_{b}^{3}=-p_{0}(k), \quad h_{t}^{3}=\frac{1}{2}\left[\left(-p_{0}+2 \mu u_{1}^{3}\right)(k+1)+\left(-p_{0}+2 \mu u_{1}^{3}\right)(k-1)\right], \\
h_{t}^{\alpha}=\frac{1}{2} \mu\left[\left(u_{1}^{\alpha}+a^{\alpha \beta} \stackrel{*}{\nabla}_{\beta} u_{0}^{3}-b_{\beta}^{\alpha} u_{0}^{\beta}\right)(k+1)+\left(u_{1}^{\alpha}+a^{\alpha \beta} \stackrel{*}{\nabla}_{\beta} u_{0}^{3}-b_{\beta}^{\alpha} u_{0}^{\beta}\right)(k-1)\right],
\end{array}\right. \\
& \left\{\begin{aligned}
-\frac{\delta^{2}}{2} p_{1} & =\left(\delta-\frac{2 H \delta^{2}}{2}\right) p_{0}+\frac{\mu \delta^{2}}{2} \beta_{0}\left(\mathbf{u}_{0}\right)+\frac{\mu \delta^{2}}{2} \stackrel{*}{\Delta} u_{0}^{3}+\frac{\delta^{2}}{2}\left(u_{0}^{\lambda} \stackrel{*}{\nabla}_{\lambda} u_{0}^{3}+b_{\alpha \beta} u_{0}^{\alpha} u_{0}^{\beta}\right)+F_{3}^{1}, \\
u_{1}^{3}=-\gamma_{0}\left(\mathbf{u}_{0}\right), & \\
\mu \delta u_{1}^{\beta}= & \frac{\mu \delta^{2}}{2}\left[\left(\stackrel{*}{\Delta} u_{0}^{\alpha}+a^{\alpha \lambda} \stackrel{*}{\nabla}_{\lambda} \operatorname{div}^{*} \mathbf{u}_{0}+K u_{0}^{\alpha}\right)+4 a^{\alpha \lambda}{ }^{*}{ }_{\lambda} H u_{0}^{3}+2 b^{\alpha \beta} \stackrel{*}{\nabla}_{\beta} u_{0}^{3}\right] \\
& -\frac{\delta^{2}}{2} a^{\alpha \beta} \stackrel{*}{\nabla}_{\beta} p_{0}+\frac{\delta^{2}}{2}\left(u_{0}^{\lambda} \stackrel{*}{\nabla}_{\lambda} u_{0}^{\alpha}-2 b_{\beta}^{\alpha} u_{0}^{\beta} u_{0}^{3}\right)+a^{\alpha \beta} F_{\beta}^{1},
\end{aligned}\right.
\end{aligned}
$$

Calculation of Drag Assume that

$$
\begin{gathered}
\mathbf{v}_{\infty}=-v_{\infty} \mathbf{k}, \quad v_{\infty}^{\alpha}=-a^{\alpha \alpha} v_{\infty} \mathbf{k} \cdot \mathbf{e}_{\alpha}, \quad v_{\infty}^{1}=0, \quad v_{\infty}^{2}=-\frac{v_{\infty}}{a_{22}} \gamma \cos \theta, \\
v_{\infty}^{3}=-v_{\infty} \mathbf{k} \cdot \mathbf{n}=\frac{v_{\infty}}{\sqrt{a}} \alpha \beta \cos \theta \sin \theta=v_{\infty} \frac{\alpha \beta}{\sqrt{a_{0}}} \cos \theta, \\
F_{d}=\frac{1}{\left|\mathbf{v}_{\infty}\right|} \int_{D}\left\{-p_{0} v_{\infty}^{3}+\mu a_{\alpha \beta} u_{1}^{\alpha} v_{\infty}^{\beta}\right\} \sqrt{a} \mathrm{~d} x=-\frac{1}{2} \int_{0}^{\pi} \int_{0}^{2 \pi} \sin (2 \theta)\left[\alpha \beta p_{0}(\varphi, \theta)+\mu \gamma \sqrt{a_{0}} u_{1}^{2}(\varphi, \theta)\right] \mathrm{d} \varphi \mathrm{d} \theta,
\end{gathered}
$$

(iii) Axial symmetry Case. If $\alpha=\beta$, then boundary layer Equation (7.23) is axial symmetry with $z$-axes. Indeed, in this case,

$$
\begin{aligned}
& a_{11}=\alpha^{2} \cos ^{2} \theta+\gamma^{2} \sin ^{2} \theta, \quad a_{12}=0, \quad a_{22}=\alpha^{2} \sin ^{2} \theta, \\
& a^{11}=\frac{1}{a_{11}}, \quad a^{22}=\frac{1}{a_{22}}, \quad a^{12}=a^{21}=0, \quad \Lambda(\varphi)=\alpha^{2}, \\
& a=a_{0} \sin ^{2} \theta, \quad a_{0}=\alpha^{2}\left(\alpha^{2} \cos ^{2} \theta+\gamma^{2} \sin ^{2} \theta\right)=\alpha^{2} a_{11}, \\
& b_{11}=\frac{\alpha \gamma \sin ^{2} \theta}{\sqrt{\alpha^{2} \cos ^{2} \theta+\gamma^{2} \sin ^{2} \theta}}, \quad b_{22}=b_{11}, \quad b_{12}=0, \\
& b^{11}=\frac{1}{b_{11}}, \quad b^{22}=\frac{1}{b_{22}}, \quad b=\operatorname{det}\left(b_{\alpha \beta}\right)=\frac{\alpha^{2} \gamma^{2}}{\alpha^{2} \cos ^{2} \theta+\gamma^{2} \sin ^{2} \theta} \sin ^{4} \theta, \\
& b_{1}^{1}=\frac{b_{11}}{a_{11}}=\frac{\alpha \gamma \sin ^{2} \theta}{\left(\alpha^{2} \cos ^{2} \theta+\gamma^{2} \sin ^{2} \theta\right)^{3 / 2}}, \\
& b_{2}^{2}=\frac{\gamma}{\alpha} \frac{1}{\sqrt{\alpha^{2} \cos ^{2} \theta+\gamma^{2} \sin ^{2} \theta}}, \\
& b_{2}^{1}=b_{1}^{2}=0, \\
& H=\frac{\gamma}{2} \frac{\alpha^{2}+\gamma^{2} \sin ^{2} \theta}{\left(\alpha^{2} \cos ^{2} \theta+\gamma^{2} \sin ^{2} \theta\right)^{3 / 2}}, \\
& K=\frac{\gamma^{2} \sin ^{2} \theta}{\left(\alpha^{2} \cos ^{2} \theta+\gamma^{2} \sin ^{2} \theta\right)^{2}},
\end{aligned}
$$


The covariant derivatives become

$$
\begin{aligned}
& \stackrel{*}{\nabla}_{1} u^{1}=\partial_{\theta} u^{1}+\frac{1}{2} \frac{\left(\gamma^{2}-\alpha^{2}\right) \sin 2 \theta}{\left(\alpha^{2} \cos ^{2} \theta+\gamma^{2} \sin ^{2} \theta\right)} u^{1}, \quad \stackrel{*}{2}_{2} u^{1}=-\frac{\alpha^{2} \sin 2 \theta}{\left(\alpha^{2} \cos ^{2} \theta+\gamma^{2} \sin ^{2} \theta\right)} u^{2}, \\
& \stackrel{*}{\nabla}_{1} u^{2}=\partial_{\theta} u^{2}+\cot \theta u^{2}, \quad \stackrel{*}{\nabla} 2 u^{2}=\cot \theta u^{1} \\
& \operatorname{div} \mathbf{u}=\partial_{\theta} u^{1}+\frac{\gamma^{2} \sin 2 \theta+\alpha^{2} \cot \theta \cos 2 \theta}{a_{11}} u^{1} \\
& \left\{a^{11} \partial_{\theta} \operatorname{div} \mathbf{u}=\frac{1}{a_{11}} \frac{\partial^{2} u^{1}}{\partial \theta^{2}}+d_{1}^{11} \frac{\partial u^{1}}{\partial \theta}+d_{1}^{1} u^{1}\right. \\
& d_{1}^{11}:=\frac{1}{a_{11}^{2}}\left[\gamma^{2} \sin 2 \theta+\alpha^{2} \cot \theta \cos 2 \theta\right] \\
& d_{1}^{1}:=\frac{1}{a_{11}^{2}}\left[-2 \gamma^{4} \sin ^{2} \theta\left(1+\cos ^{2} \theta\right)+\alpha^{4} \cos ^{2} \theta\left(2 \cos 2 \theta-\cot ^{2} \theta\right)+\alpha^{2} \gamma^{2} \sin 2 \theta \cot \theta\left(2 \cos ^{2} \theta-3 \sin ^{2} \theta\right)\right], \\
& B^{1}(\mathbf{u}, \mathbf{u})=u^{\lambda} \stackrel{*}{\nabla}_{\lambda} u^{1}-u^{1} \operatorname{div} \mathbf{u}=-\frac{1}{a_{11}}\left[\left(\gamma^{2} \sin \theta \cos \theta+\alpha^{2} \cot \theta \cos \theta\right) u^{1} u^{1}+\alpha^{2} \sin 2 \theta u^{2} u^{2}\right], \\
& B^{2}(\mathbf{u}, \mathbf{u})=u^{\lambda} \stackrel{*}{\nabla} \lambda u^{2}-u^{2} \operatorname{div}^{*} \mathbf{u}=u^{1} \partial_{\theta} u^{2}+\cot \theta\left(u^{1} u^{1}+u^{2} u^{1}\right) \\
& \stackrel{*}{\Delta} p_{0}=\frac{1}{a_{11}} \frac{\partial^{2} p_{0}}{\partial \theta^{2}}-\frac{\alpha^{2} \ln \alpha}{a_{11}} \sin 2 \theta \partial_{\theta} p_{0} \\
& \left\{\begin{array}{l}
*^{*} u^{1}=\frac{1}{a_{11}} \frac{\partial^{2} u^{1}}{\partial \theta^{2}}+L_{1}^{11} \partial_{\theta} u^{1}+L_{1}^{1} u^{1}, \quad \stackrel{*}{\Delta} u^{2}=\frac{1}{a_{11}} \frac{\partial^{2} u^{2}}{\partial \theta^{2}}+L_{2}^{21} \partial_{\theta} u^{2}+L_{2}^{2} u^{2} \\
L_{1}^{11}:=-\frac{1}{a_{11}^{2}}\left(\gamma^{2} \sin \theta \cos \theta+\alpha^{2} \cot \theta(1+\cos \theta)\right) \\
L_{1}^{1}:=\frac{\gamma^{2}-\alpha^{2}}{a_{11}^{3}} \cos ^{2} \theta\left[\alpha^{2} \cos ^{2} \theta-\gamma^{2}\left(3 \sin ^{2} \theta-1\right)\right] \\
L_{2}^{21}:=\frac{\cot \theta}{\alpha^{4} a_{11}}\left[3 \alpha^{4}+\alpha^{2} \gamma^{2}-\gamma^{4}+\alpha^{2}\left(\alpha^{2}-\gamma^{2}\right) \cot \theta\right] \\
L_{2}^{2}:=\frac{1}{a_{11}^{2} a_{22}}\left(\frac{\alpha^{2}\left(\gamma^{2}-\alpha^{2}\right)}{4} \sin ^{2} 2 \theta+\alpha^{2} a_{11}\right)
\end{array}\right.
\end{aligned}
$$

Let

$$
U_{\lambda}^{\alpha}:=u_{\lambda}^{\alpha},\left(U_{\lambda}^{\alpha}\right)^{\prime}=\partial_{\theta} U_{\lambda}^{\alpha},\left(U_{\lambda}^{\alpha}\right)^{\prime \prime}=\partial_{\theta}^{2} U_{\lambda}^{\alpha}, \lambda=0,1
$$

Then BLE I (7.23) and SLE II (7.27) become

$$
\left\{\begin{array}{l}
-\frac{\mu \delta^{3}}{3}\left[\frac{2}{a_{11}}\left(U_{1}^{1}\right)^{\prime \prime}+l_{1}^{11}\left(U_{1}^{1}\right)^{\prime}+l_{1}^{1} U_{1}^{1}\right]+\frac{Q_{11}}{a_{11}} U_{1}^{1}+\frac{\delta^{4}}{4} B^{1}\left(\mathbf{u}_{1}, \mathbf{u}_{1}\right)+d^{11} \partial_{\theta} p_{0}-\frac{4 \delta^{3}}{3} \frac{1}{a_{11}} \partial_{\theta}\left(H p_{0}\right) \\
=\frac{1}{a_{11}}\left[\mathscr{F}^{1}-\frac{\delta^{3}}{3} \partial_{\theta} p_{1}\right], \\
-\frac{\mu \delta^{3}}{3}\left[\frac{1}{a_{11}}\left(U_{1}^{2}\right)^{\prime \prime}+l_{2}^{21}\left(U_{1}^{2}\right)^{\prime}+l_{2}^{2} U_{1}^{2}\right]+\frac{Q_{22}}{a_{22}} U_{1}^{2}+\frac{\delta^{4}}{4} B^{2}\left(\mathbf{u}_{1}, \mathbf{u}_{1}\right)=\frac{1}{a_{22}} \mathscr{F}_{2}^{1}, \\
\frac{1}{a_{11}} \frac{\partial^{2} p_{0}}{\partial \theta^{2}}-\frac{\alpha^{2} \ln \alpha}{a_{11}} \sin 2 \theta \partial_{\theta} p_{0}+m_{1}^{1} \frac{\partial u_{1}^{1}}{\partial \theta}+m_{1} u_{1}^{1}+m_{2} u_{1}^{2}=F_{p},
\end{array}\right.
$$


where

$$
\left\{\begin{array}{l}
l_{1}^{11}=L_{1}^{11}+d_{1}^{11}=-\frac{1}{a_{11}^{2}}\left[a_{11}^{2} \cot \theta+\gamma^{2} \sin \theta \cos \theta+\alpha^{2} \cot \theta(1+\cos \theta)\right] \\
l_{1}^{1}=L_{1}^{1}+d_{1}^{1}+K=\frac{\gamma^{2}-\alpha^{2}}{a_{11}^{3}} \cos ^{2} \theta\left[\alpha^{2} \cos ^{2} \theta-\gamma^{2}\left(3 \sin ^{2}-1\right)\right] \\
+\frac{1}{a_{11}^{2}}\left[-2 \gamma^{4} \sin ^{2} \theta\left(1+\cos ^{2} \theta\right)+\alpha^{4} \cos ^{2} \theta\left(2 \cos 2 \theta-\cot ^{2} \theta\right)+\alpha^{2} \gamma^{2} \sin 2 \theta \cot \theta\left(2 \cos ^{2} \theta-3 \sin ^{2} \theta\right)+\gamma^{2} \sin ^{2} \theta\right] \\
l_{2}^{2}=L_{2}^{2}+K=\frac{\alpha^{2}}{a_{11}^{2} a_{22}}\left(\frac{1}{4} \alpha^{2}\left(\gamma^{2}-\alpha^{2}\right) \sin ^{2} 2 \theta+\alpha^{2} a_{11}+\gamma^{2} \alpha^{2} \sin ^{2} \theta\right) \\
Q_{\alpha \beta}=2 \mu\left[\left(\frac{1}{8} \delta+\frac{3}{2} \frac{H \delta^{2}}{2}+\left(4 K-2 H^{2}\right) \frac{\delta^{3}}{3}\right) a_{\alpha \beta}+\left(\frac{\delta^{2}}{2}-\frac{11 H \delta^{3}}{3}\right) b_{\alpha \beta}\right] \\
m_{1}^{1}:=2 \mu\left(\frac{\delta^{2}}{2}-\frac{2 b_{2}^{2} \delta^{3}}{3}\right) u_{1} \\
:=\left[\frac{\mu \delta^{2}}{2}\left(\frac{\gamma^{2}-\alpha^{2}}{a_{11}} \sin 2 \theta+\cot \theta\right)+\frac{6 \mu \delta^{3}}{3} \partial_{\theta} H-\frac{4 \mu \delta^{3}}{3}\left(\frac{\gamma \alpha^{2}}{a_{11}^{3 / 2}}\left(\alpha^{2}-\gamma^{2} \cos \theta\right) \cos ^{2} \theta\right)\right] \\
m_{2}:=\frac{6 \mu \delta^{3}}{3} \partial_{\theta} H u_{1}^{2}
\end{array}\right.
$$

The drag is given by

$$
F_{d}=-\pi \int_{0}^{\pi} \sin (2 \theta)\left[\alpha^{2} p_{0}(\theta)+\mu \alpha \gamma \sqrt{a_{22}} U^{2}(\theta)\right] \mathrm{d} \theta
$$

In the following we concern with the axi-symmetric flow around an ellipsoid, which depends significantly on the Reynolds number and the geometry of the ellipsoid. And the boundary layer equations are solved with spectral method. The fluid approaches the ellipsoid with a uniform free-stream velocity from inlet to outlet. In order to compare with the results in reference conveniently, the results should be dimensionless. Therefore the other parametric equation of ellipsoid is proposed

$$
\xi_{0}=\tanh ^{-1}(\alpha / \gamma), \quad \alpha=\beta=c \cosh \xi_{0}, \quad \gamma=c \sinh \xi_{0}
$$

where $c$ a constant and the parameter $\xi_{0}$ defines the surface of the spheroid and is related to the axis ratio by $\xi_{0}$. A perfect sphere would be represented by $\xi_{0} \rightarrow \infty$ whereas a flat circular disk would be represented by $\xi_{0}=0$.

The Reynolds number based on the focal length, i.e. $\operatorname{Re}=\frac{2 c \rho U_{\infty}}{\mu}$, varies from 0.1 to 1.0. In the case the focal length $2 \gamma$ is the reference length and inlet velocity $U_{\infty}$ is the reference velocity. Let the total drag coefficient be,

$$
C_{D}=\frac{F_{d} C}{A \mu U_{\infty}}
$$

where $A=\pi c^{2} \cosh ^{2} \xi_{0}$ is the spheroid projected area. From BLE I the total drag includes two terms and the first term is the pressure part while the second term is the viscous part, i.e. $F_{d}=F_{d p}+F_{d v}$, which are defined as,

$$
\begin{aligned}
& F_{d p}=-\pi \int_{0}^{\pi} \sin (2 \theta)\left[\alpha^{2} p_{0}(\theta)\right] \mathrm{d} \theta, \\
& F_{d v}=-\pi \int_{0}^{\pi} \sin (2 \theta)\left[\mu \alpha \gamma \sqrt{a_{22}} U^{2}(\theta)\right] \mathrm{d} \theta,
\end{aligned}
$$

Therefore the total drag coefficient is also decomposed into pressure and viscous part: $C_{D}=C_{D V}+C_{D P}$, in which,

$$
C_{D V}=\frac{F_{d v}}{A \mu U_{\infty}}, C_{D P}=\frac{F_{d p}}{A \mu U_{\infty}},
$$


Firstly the numerical solution of boundary layer equations is validated quantitatively by comparison with results in references and finite element method. Table 1 presents results of pressure and total drag coefficients for various Reynolds numbers at $\xi_{0}=0.5$. Table 2 presents results of pressure and total drag coefficients for various values of $\xi_{0}$ at $\mathrm{Re}=1.0$. An excellent agreement between the present results and that of Alassar and Badr [13] are both achieved. And the normal stress tensor $\left.h\right|_{\mathfrak{\Im}(\delta)}$ to the supper surface of boundary layer is considered as the boundary condition of boundary layer equations, which is obtained from the solutions of finite element method. According to Table 1 and Table 2 the precision of drag computation with boundary layer equations is higher than the finite element method, so the boundary layer equations could be used to improve the computation precision of flow in the boundary layer with low cost.

Figure 2 presents the nearly stationary streamline patterns and pressure distributions at different Reynolds numbers 10, 30, 60 and 100 respectively for $\xi_{0}=0.5$. Here we note that our streamline patterns are similar to those obtained by Rimon and Cheng [14] for the sphere, since the separation angles and wake lengths are in close agreement with each other. Figure 2(b) shows a clearly visible secondary vortex at $\operatorname{Re}=60$, in this regard our result is also consistent with Rimon and Cheng's [14] in spite of the difference in the size of the wake. Furthermore, Figure 2(d) shows a nice structure which corresponds to the a phenomenon observed for the flow around a circular cylinder. Since secondary vortices appear only at relatively high Reynolds number, we may conclude that the wake region is much more active at higher Reynolds number rather than that the wake length has to increase with the Reynolds number.

Figure 3 presents the nearly stationary streamline patterns and pressure distributions at different $\xi_{0} \quad 0.25,0.5$, 1.0 and 1.5 respectively for $\operatorname{Re}=1.0$. As expected, no separation occurs at the low Re values.

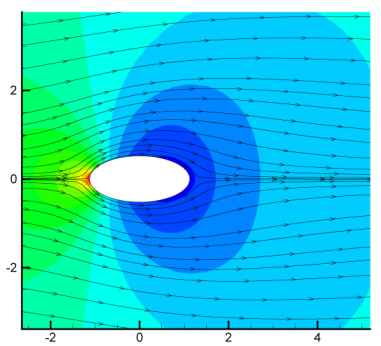

(a)

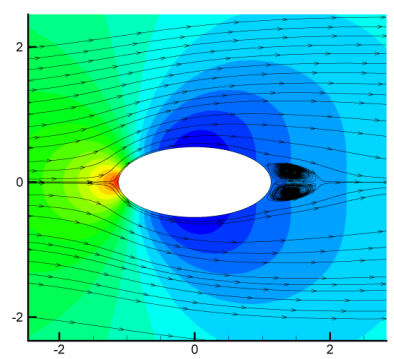

(c)

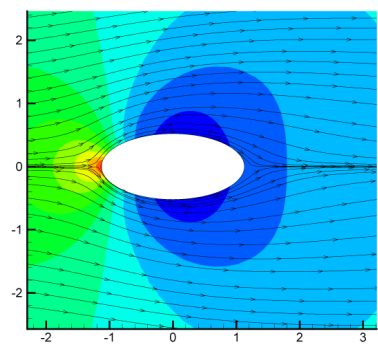

(b)

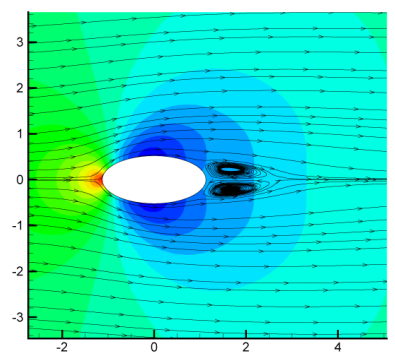

(d)

Figure 2. Streamlines of the flow:(a) $\operatorname{Re}=10$; (b) $\operatorname{Re}=30$; (c) $\operatorname{Re}=60$; (d) $\operatorname{Re}=100$ for $\xi_{0}=0.5$.

Table 1. Comparison of drag coefficients for various Reynolds numbers at $\xi_{0}=0.5$.

\begin{tabular}{ccccccc}
\hline & \multicolumn{3}{c}{$C_{D}$} & & & \multicolumn{2}{c}{$C_{D P}$} \\
\cline { 3 - 7 } & Ref. [13] & FEM & BLE & Ref. [13] & FEM & BLE \\
\hline 0.1 & 4.8934 & 4.9518 & 4.8734 & 2.6866 & 2.7615 & 2.6754 \\
0.5 & 5.1638 & 5.3143 & 5.2036 & 2.8363 & 2.9146 & 2.8562 \\
1.0 & 5.4700 & 5.6147 & 5.5813 & 3.0075 & 3.1164 & 3.0819 \\
\hline
\end{tabular}


Then the flow details around the trailing edge of ellipsoid for $\operatorname{Re}=60, \xi_{0}=0.5$ are given in Figure 4. It is obvious that the secondary vortex appears in the result of BLE, so more details could be computed by BLE than FEM. Although these flow details is obtained by FEM, its computational cost would be much more expensive than BLE. Let dimensionless pressure be $p^{*}(\theta)=p^{\prime}(\theta)-p^{\prime}(\pi)$ and the definition of $p^{\prime}$ is as follows,

$$
p^{\prime}(\theta)=\frac{p(\theta) c}{\mu U_{\infty}}
$$

Figure 5 shows shows the surface dimensionless pressure distributions for the case $\xi_{0}=0.5$ when $\operatorname{Re}=10$, 30, 60 and 100. As Re increases, the difference in the pressure between the front and the rear stagnation points increases.

Figure 6 proposes the corresponding pressure distributions in 3D.

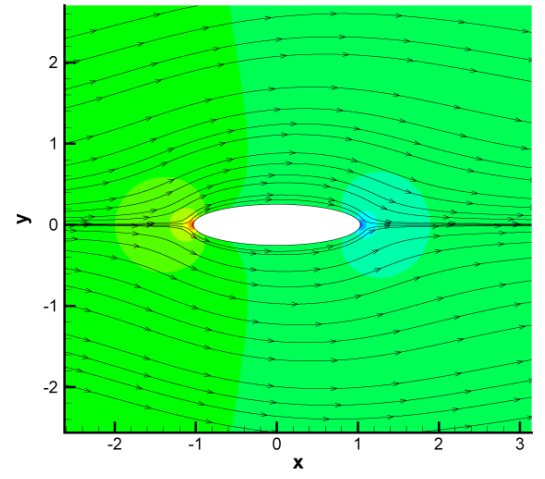

(a)

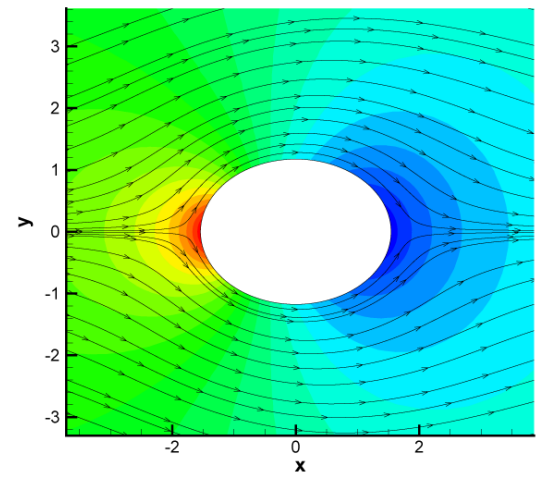

(c)

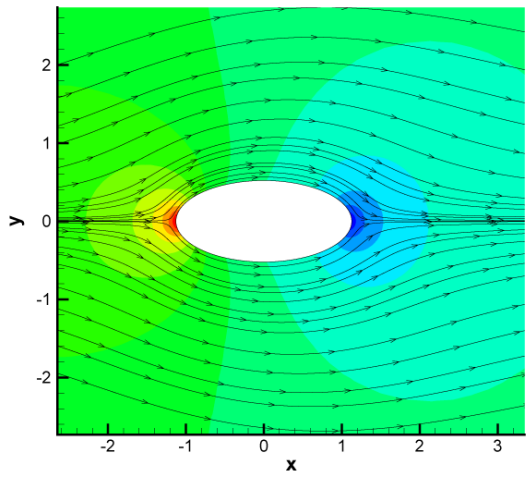

(b)

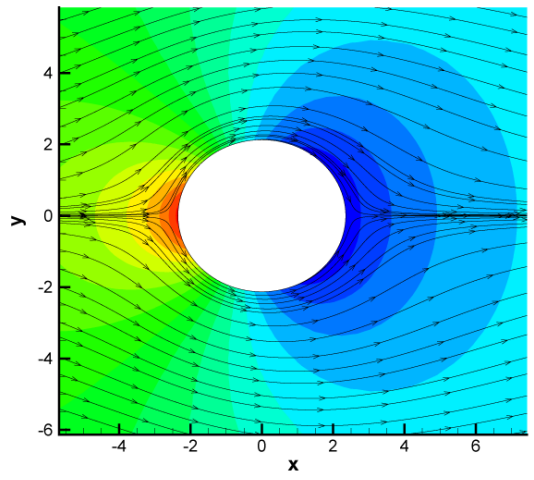

(d)

Figure 3. Streamlines of the flow: (a) $\xi=0.25$; (b) $\xi=0.5$; (c) $\xi=1.0$; (d) $\xi=1.5$ for $\operatorname{Re}=1.0$.

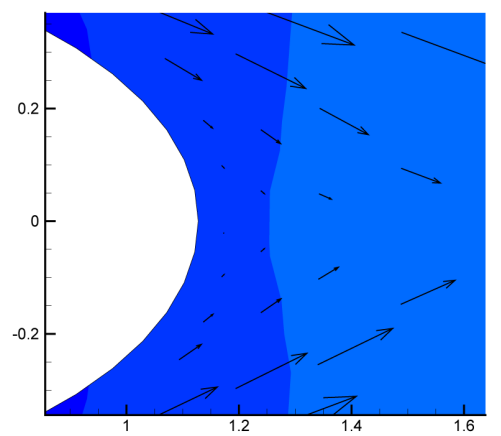

(a)

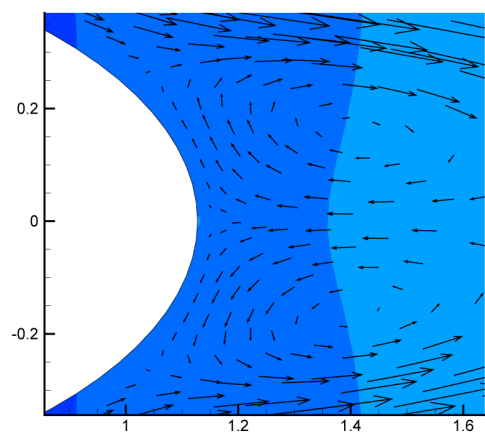

(b)

Figure 4. Comparison of flow details for $\operatorname{Re}=60, \xi_{0}=0.5$ : (a) FEM; (b) BLE. 


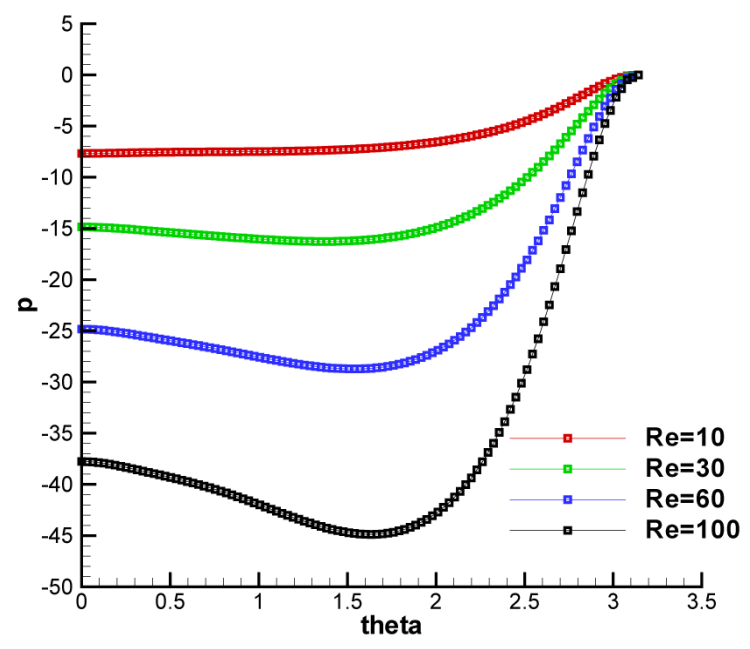

Figure 5. Surface pressure distribution for $\xi_{0}=0.5$.

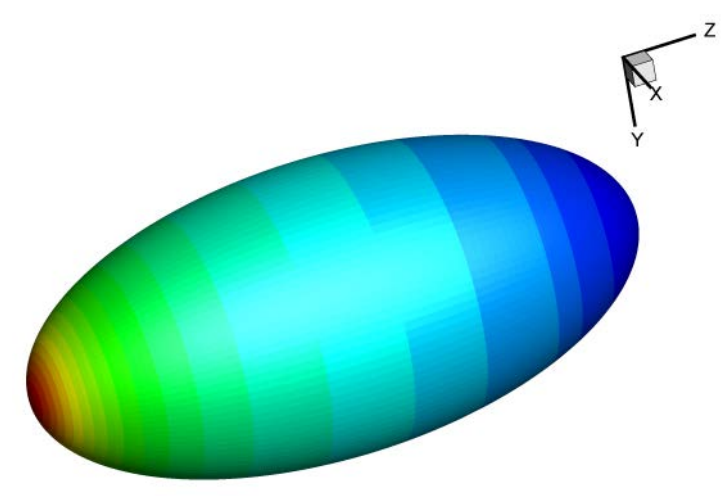

p: $-8-8-7-7-7-7-6-6-5-5-4-4-3-3-2-2-1-10$

(a)

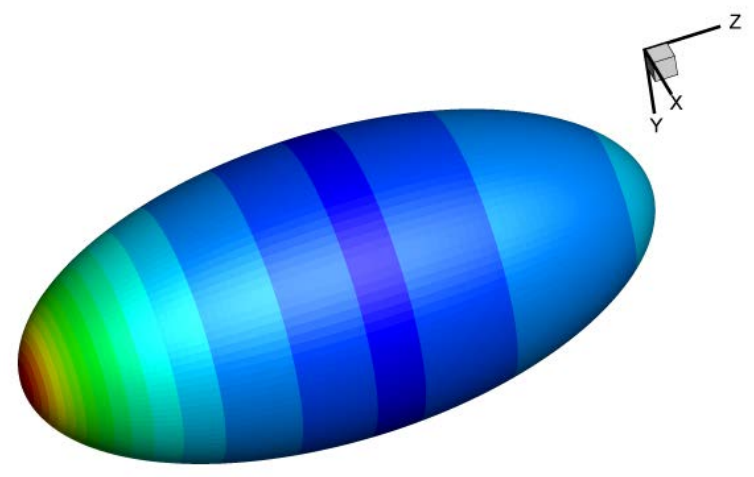

p: $-29-28-26-24-22-20-18-16-14-12-10-8 \quad-6 \quad-4 \quad-2$

(c)

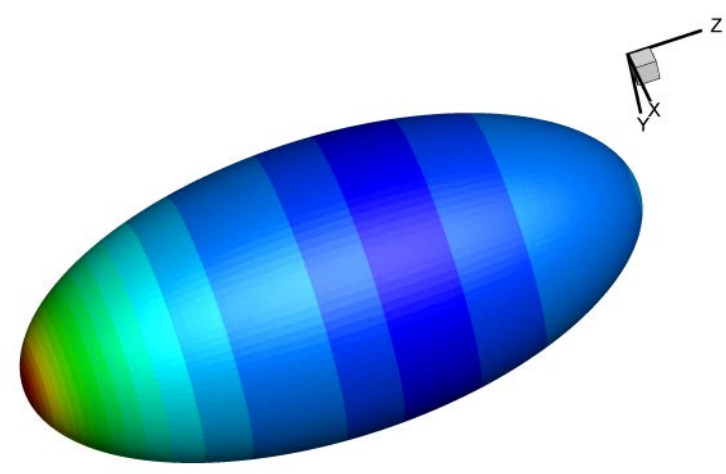

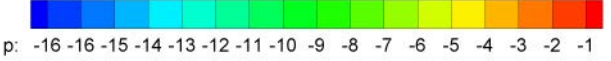

(b)

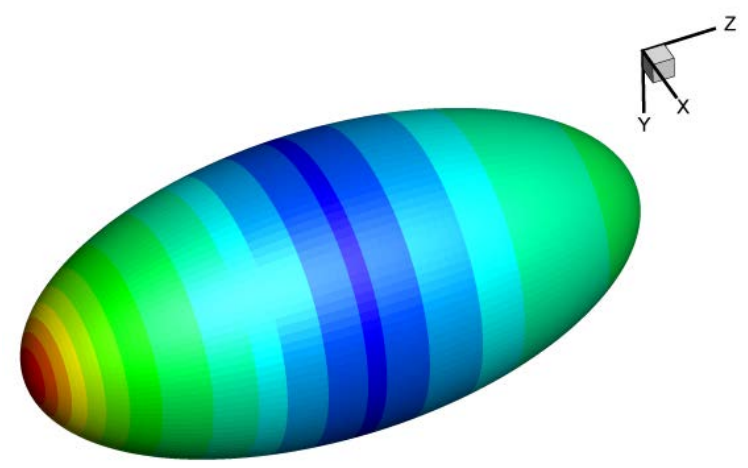

p: $\begin{array}{rlllll}-44.867 & -43.6574 & -40 & -30 & -20 & -10\end{array}$

(d)

Figure 6. Surface pressure distribution in 3D: (a) $\operatorname{Re}=10$; (b) $\operatorname{Re}=30$; (c) $\operatorname{Re}=60$; (d) $\operatorname{Re}=100$ for $\xi_{0}=0.5$. 
The effect of $\xi_{0}$ on the pressure distribution can be seen in Figure 7. The figure which show the results at $\operatorname{Re}=1.0$ when $\xi_{0}=0.25,0.5,1.0$ and 1.5 indicates that when $\xi_{0}$ decreases, a positive pressure gradient may be expected. The surface pressure distributions are compared between FEM and BLE in Figure 8 for the case $\operatorname{Re}=1.0$ when $\xi_{0}=0.25,0.5,1.0$ and 1.5. The pressure distributions obtained by FEM and BLE are almost the same, however the absolute value of pressure in FEM is generally a little higher than these in BLE, which is consistent with the results in Table 2.

Figure 9 proposes the corresponding pressure distributions in 3D.

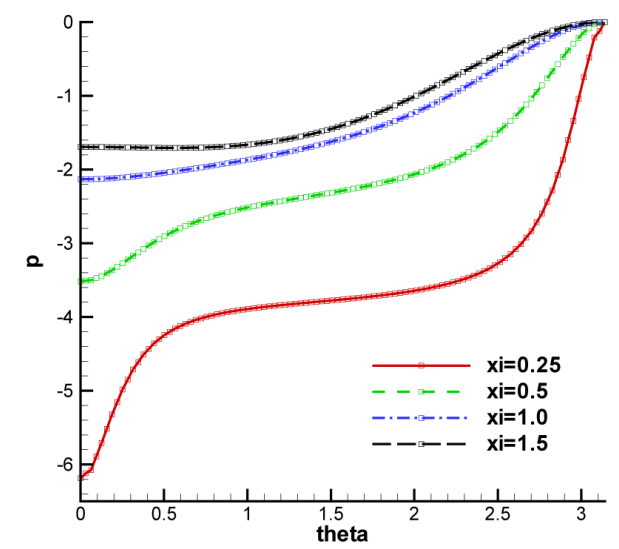

Figure 7. Surface pressure distribution for $\operatorname{Re}=1.0$.

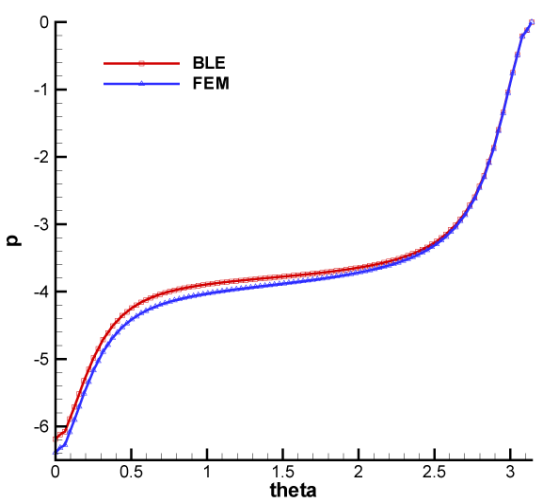

(a)

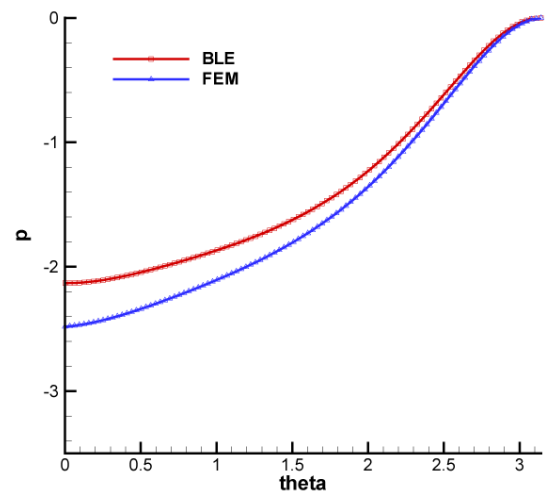

(c)

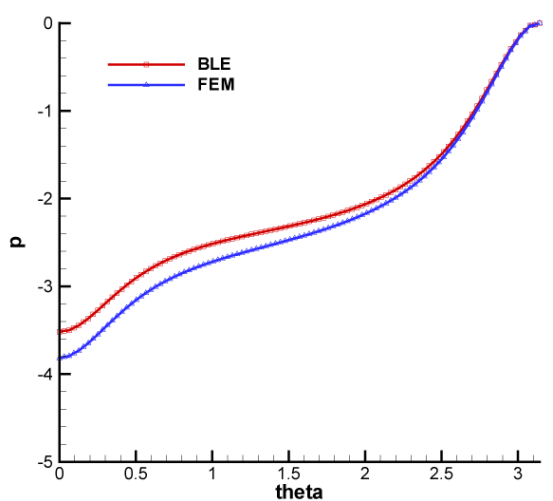

(b)

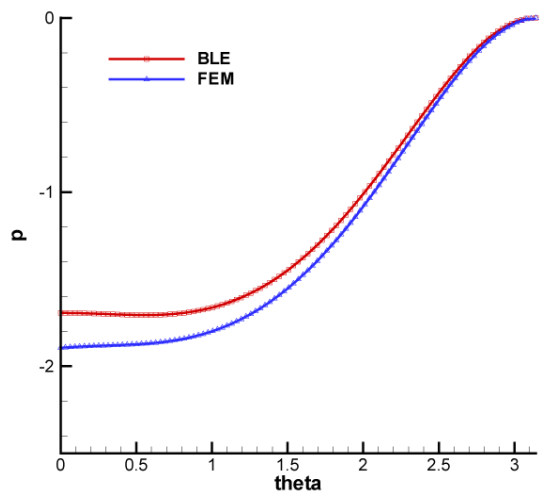

(d)

Figure 8. Comparison of surface pressure distribution between FEM and BLE: (a) $\xi_{0}=0.25$;

(b) $\xi_{0}=0.5$; (c) $\xi_{0}=1.0$; (d) $\xi_{0}=1.5$ for $\operatorname{Re}=1.0$. 

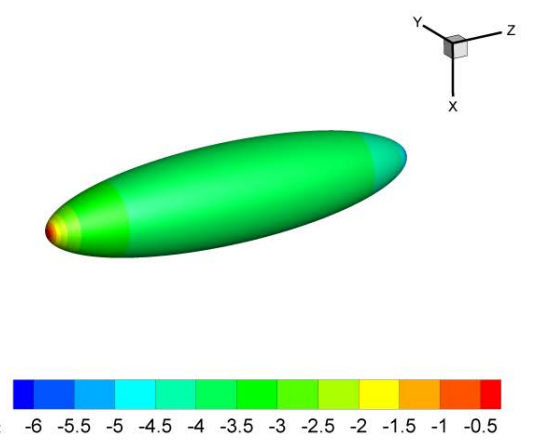

(a)
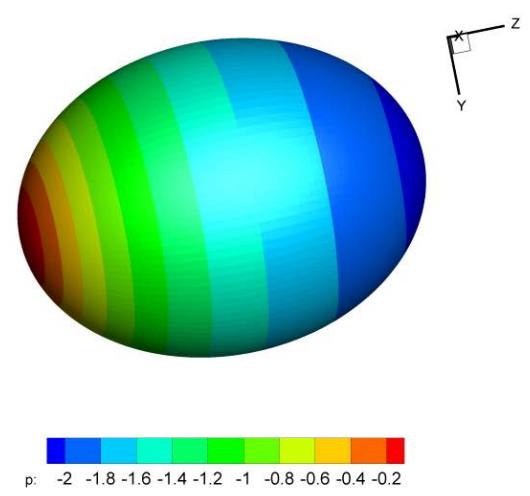

(c)

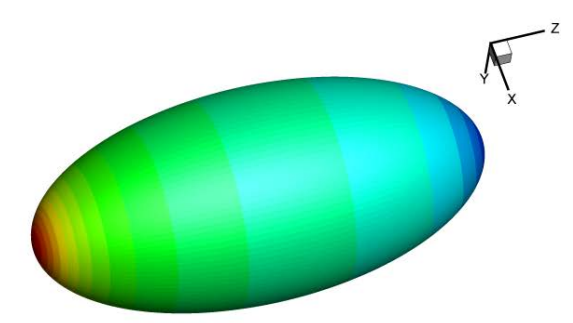

p: $-3.4-3.2-3-2.8-2.6-2.4-2.2-2-1.8-1.6-1.4-1.2-1 \quad-0.8-0.6-0.4-0.2$

(b)

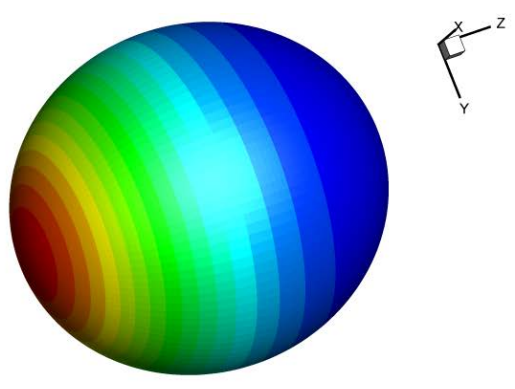

$-1.6-1.5-1.4-1.3-1.2-1.1-1-0.9-0.8-0.7-0.6-0.5-0.4-0.3-0.2-0.1$

(d)

Figure 9. Surface pressure distribution in 3D: (a) $\xi=0.25$; (b) $\xi=0.5$; (c) $\xi=1.0$; (d) $\xi=1.5$ for $\operatorname{Re}=1.0$.

Table 2. Comparison of drag coefficients for various values of $\xi_{0}$ at $R e=1.0$.

\begin{tabular}{ccccccc}
\hline$\xi_{0}$ & \multicolumn{3}{c}{$C_{D}$} & & \multicolumn{2}{c}{$C_{D P}$} \\
\cline { 2 - 7 } & Ref. [13] & FEM & BLE & Ref. [13] & FEM & BLE \\
\hline 0.25 & 5.6995 & 5.8013 & 5.7124 & 4.0390 & 4.0928 & 4.0845 \\
0.5 & 5.4700 & 5.6123 & 5.5167 & 3.0075 & 3.0967 & 3.0616 \\
1.0 & 4.4265 & 4.5741 & 4.5638 & 1.8140 & 1.8816 & 1.8564 \\
1.5 & 3.2020 & 3.3569 & 3.2964 & 1.1635 & 1.2001 & 1.1757 \\
\hline
\end{tabular}

Finally, it has to be emphasized that since flow axisymmetry is assumed in the present study, none of our results give any indication about symmetry-breaking in a real flow. The presented method are, however, not restricted to axi-symmetric flow, the BLE I aforementioned could be used to compute the non-axisymmetric flow.

\section{Support}

Supported by Major Research Plan of NSFC (91330116), National Basic Research Program No 2011CB 706505, NSFC 11371288, 11371289.

\section{References}

[1] Li, K.T. and Huang, A.X. (2013) Boundary Shape Control of Navier-Stokes Equations and Dimensional Splitting Methods and Its Applications. Science Press, Beijing (in Chinese). 
[2] Li, K.T., Chen, H. and Yu, J.P. (2013) An New Boundary Layer Equations and Applications to Shape Control. Scientia Sinica (Mathematics), 43, 965-1021 (in Chinese). http://dx.doi.org/10.1360/012012-428

[3] Li, K.T., Su, J. and Huang, A.X. (2010) Boundary Shape Control of the Navier-Stokes Equations and Applications. Chinese Annals of Mathematics, 31B, 879-920.

[4] Ciarlet, P.G. (2000) Mathematical Elasticity, Vol. III: Theory of Shells. North-Holland, Amsterdam.

[5] Li, K.T., Zhang, W.L. and Huang, A.X. (2006) An Asymptotic Analysis Method for the Linearly Shell Theory. Science in China, Series A, 49, 1009-1047.

[6] Temam, R. and Ziane, M. (1997) Navier-Stokes Equations in Thin Spherical Domains. Contemporary Mathematics, 209, 281-314. http://dx.doi.org/10.1090/conm/209/02772

[7] Li, K.T., Yu, J.P. and Liu, D.M. (2012) A Differential Geomety Methods for Rational Navier-Stokes Equations with Complex Boundary and Two Scales Paralelell Algorithms. Acta Mathematicae Applicae Sinica, 35, 1-41.

[8] Li, K.T., Huang, A.X. and Zhang, W.L. (2002) A Dimension Split Method for the 3-D Compressible Navier-Stokes Equations in Turbomachine. Communications in Numerical Methods in Engineering, 18, 1-14.

[9] Li, K.T. and Liu, D.M. (2009) Dimension Splitting Method for 3D Rotating Compressible Navier-Stokes Equations in the Turbomachinery. International Journal of Numerical Analysis and Modeling, 6, 420-439.

[10] Li, K.T., Yu, J.P., Shi, F. and Huang, A.X. (2012) Dimension Splitting Method for the Three Dimensional Rotating Navier-Stokes Equations. Acta Mathematicae Applicatae Sinica-English Series, 28, 417-442. http://dx.doi.org/10.1007/s10255-012-0161-7

[11] Temam, R. (1984) Navier-Stokes Equations, Theorem and Numerical Ananlysis. North Holland, Amsterdam, U.S.A., New York.

[12] Giraut, V. and Raviart, P.A. (1985) Finite Element Approximation of the Navier-Stokes Equations. Springer-Verlag, Berlin.

[13] Alassar, R.S. and Badr, H.M. (1999) Oscillating Flow over Oblate Spheroids. Acta Mechanica, 137, $237-254$. http://dx.doi.org/10.1007/BF01179212

[14] Rimon, Y. and Cheng, S.I. (1969) Numerical Solution of a Uniform Flow over a Sphere at Intermediate Reynolds Numbers. Physics of Fluids, 12, 949-959. http://dx.doi.org/10.1063/1.2163685 